Supporting Information

for

\title{
Brønsted Acid-Assisted Zinc-Catalyzed Markovnikov-Type Hydrothiolation of Alkenes Using Thiols
}

\author{
Nobukazu Taniguchi* \\ Department of Chemistry, Fukushima Medical University, Fukushima 960-1295, Japan
}

Phone: $+81-245471369$

Fax: $+81-245471369$

taniguti@fmu.ac.jp

Table of Contents

Copies of ${ }^{1} \mathrm{H}$ and ${ }^{13} \mathrm{C}$ NMR Charts for the All Compounds

S2-S61 


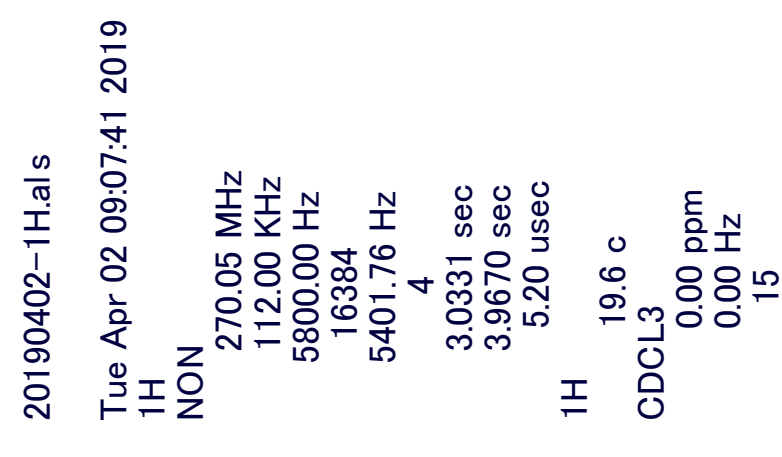

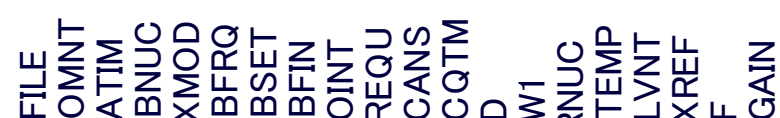

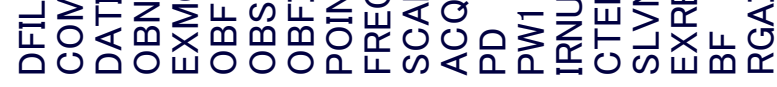

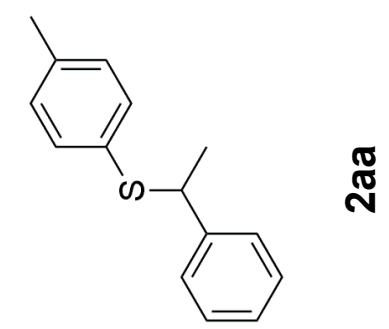

000

69.

$\mid 9$.

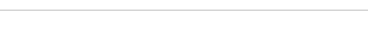

$8 z \cdot$

Z๐

sZ"

LZ゙ち

$0 \%$

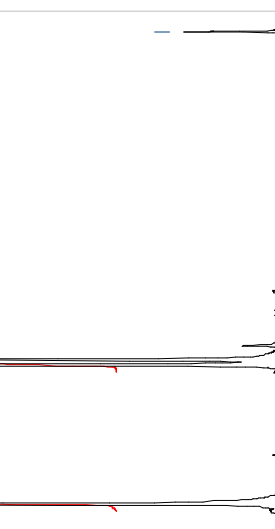

$00 \%$

$\checkmark 0^{\circ} L$

$\angle I L L$

$0 Z L$

IZL

$\varepsilon Z^{\circ} L$

8.4

$1-7$

$\nabla Z L$

$9 Z^{\circ} L$

$L Z L$ 


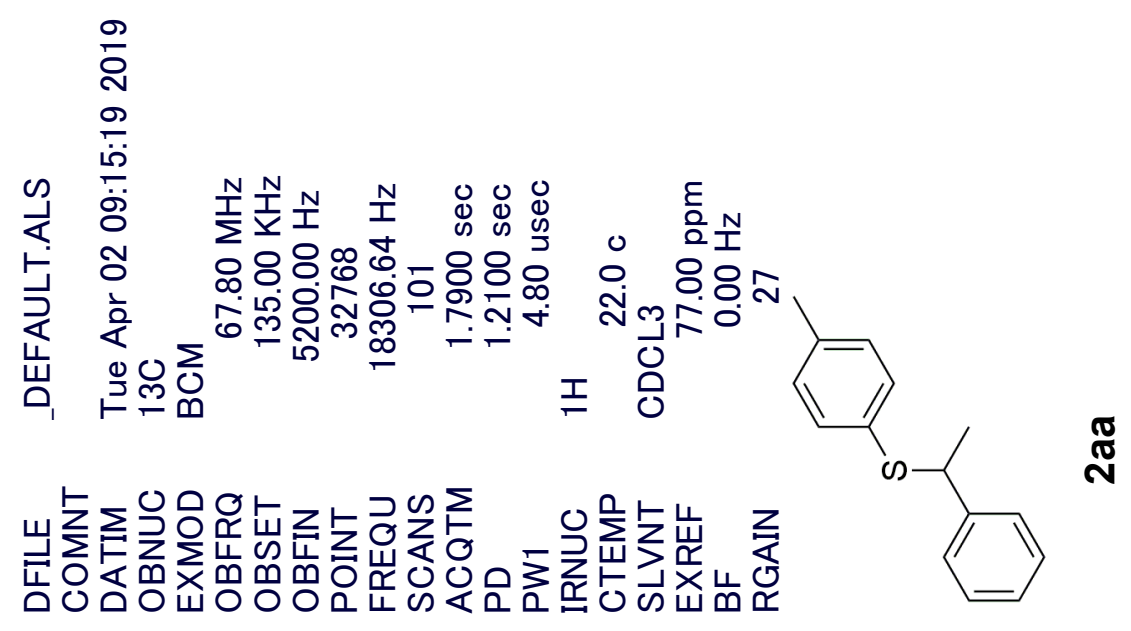

80.12
t.'2Z

乙E'8t

$\varepsilon G^{\circ} 9 L$

$00^{\circ} L L$

$\angle \nabla^{\circ} L L$

LOLZL

$9 Z$ LZL

6Z8Z1

乙†6て

乙乙 เE।

GI $\mathcal{E} \mid$

$\perp \mathcal{E}^{\circ} L \mathcal{E}$ ।

$0 \varepsilon^{\prime} \varepsilon \supset ।$

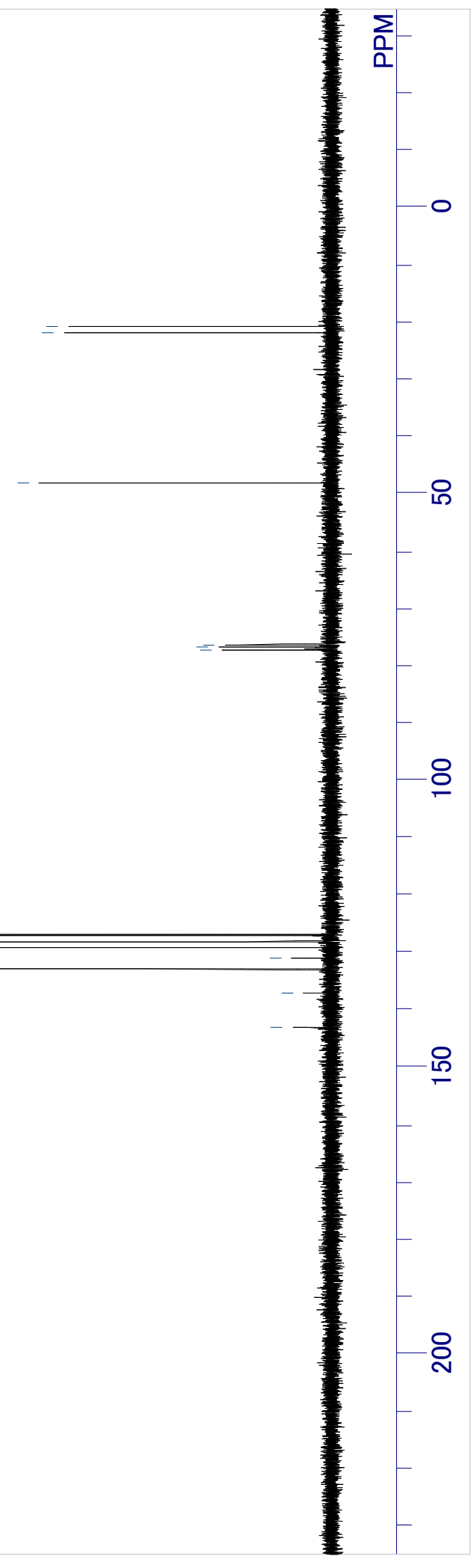




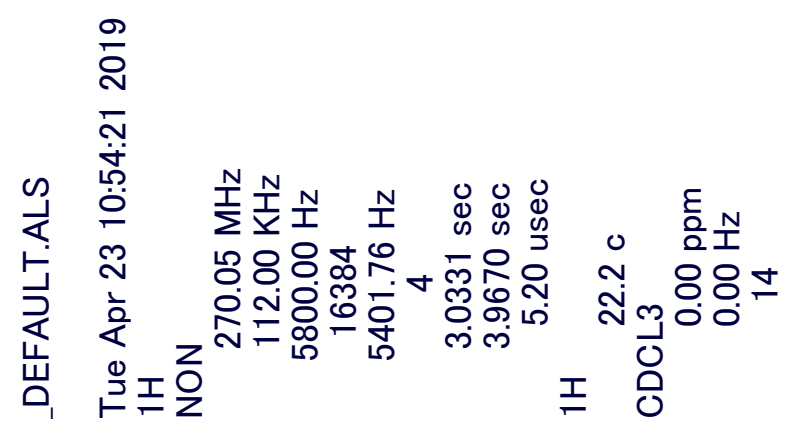

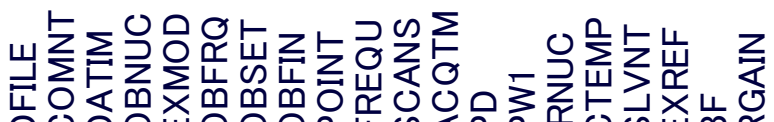

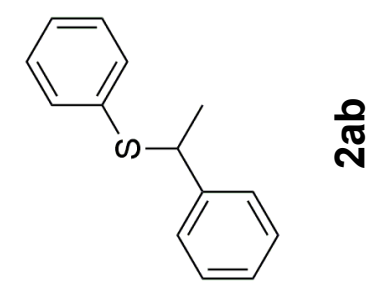

000

19.1

$\varepsilon 9$ ।

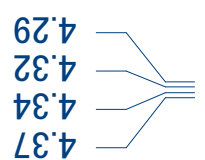

$\angle \varepsilon^{\prime} \dagger$

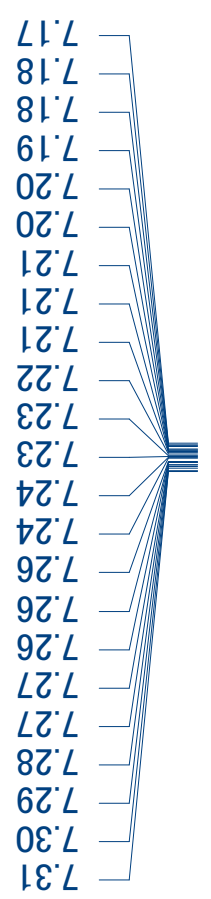




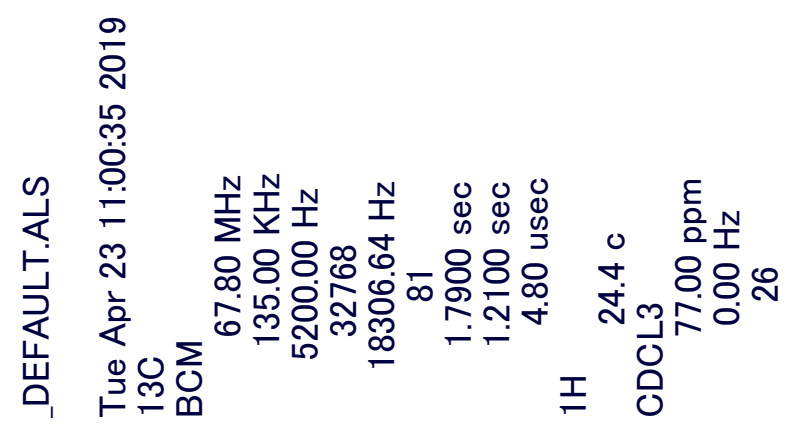

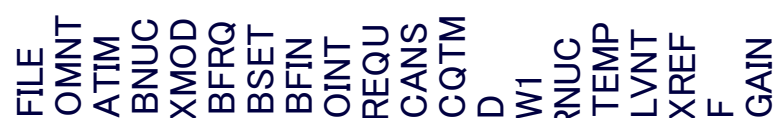

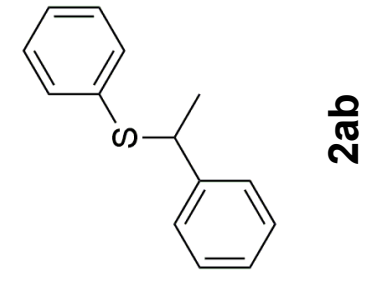

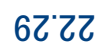

$96^{\circ} \angle t$

$\varepsilon G 9 L$

$00^{\circ} L L$

$\angle t L L$

$80^{\circ} \angle Z 1$

60 LZI

$\checkmark Z$ LZI

GE $8 Z 1$

†9 $8 \mathrm{ZL}$

G†'टा

60 다

9 |'Et|

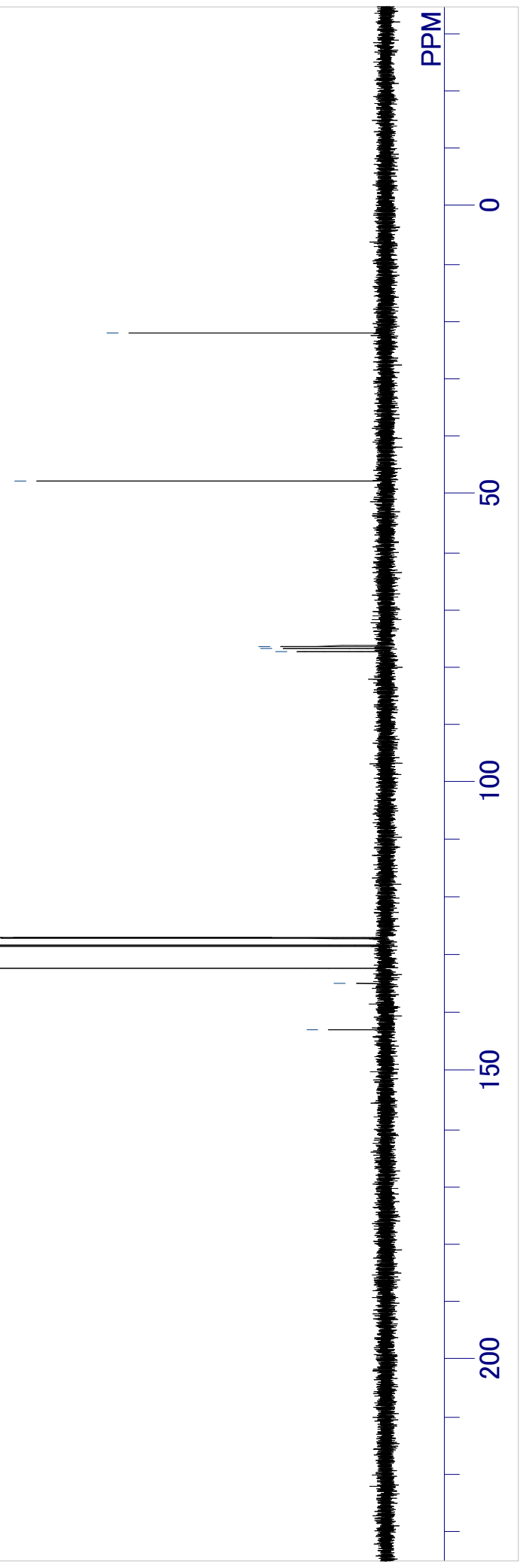




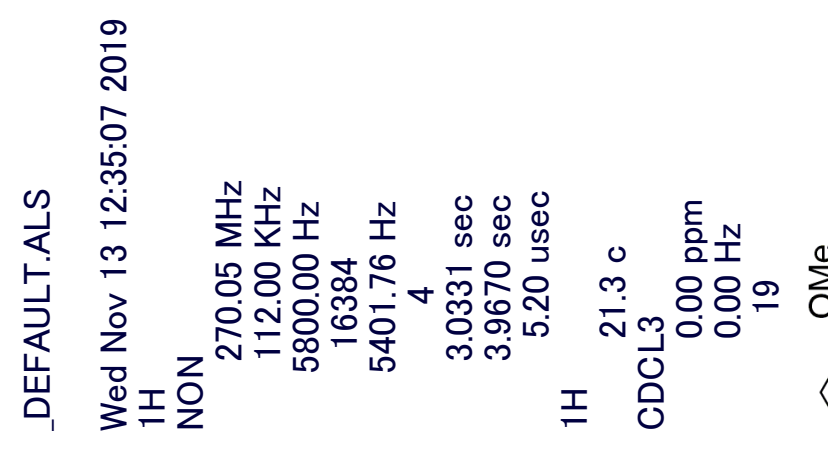

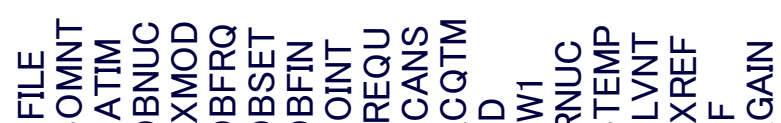

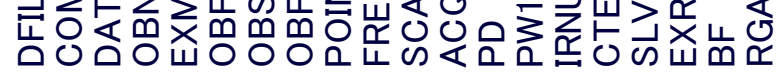<smiles>[C+]c1ccccc1</smiles>

$00^{\circ} 0$

$t c^{\circ}$

$89^{\circ}$ ।

$09^{\circ}$ I

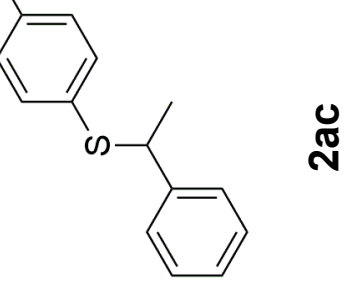

$\stackrel{\text { N }}{\mathbb{N}}$

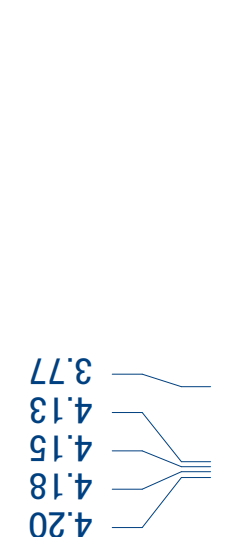

$\nabla L 9$

$\angle L ' 9$

$\angle I L$

$0 Z L$

$Z Z L$

$\varepsilon Z{ }^{\prime} L$

$G Z " L$

$G Z L$

$9 Z L$

$8 Z L$

$8 Z^{\circ} L$ 

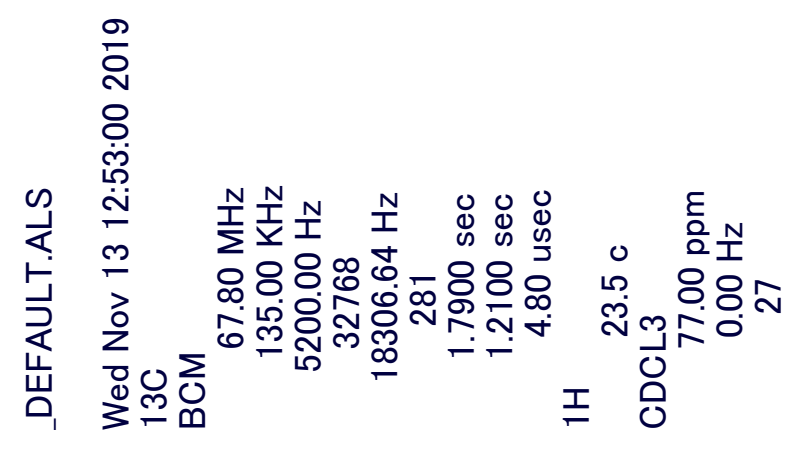

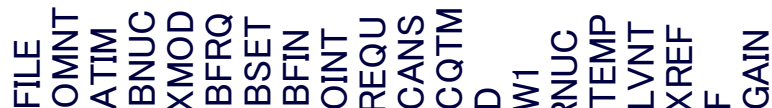<smiles>[CH2]Oc1ccccc1</smiles>

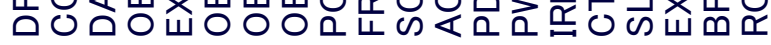

$L L^{\prime} I Z$

01.67
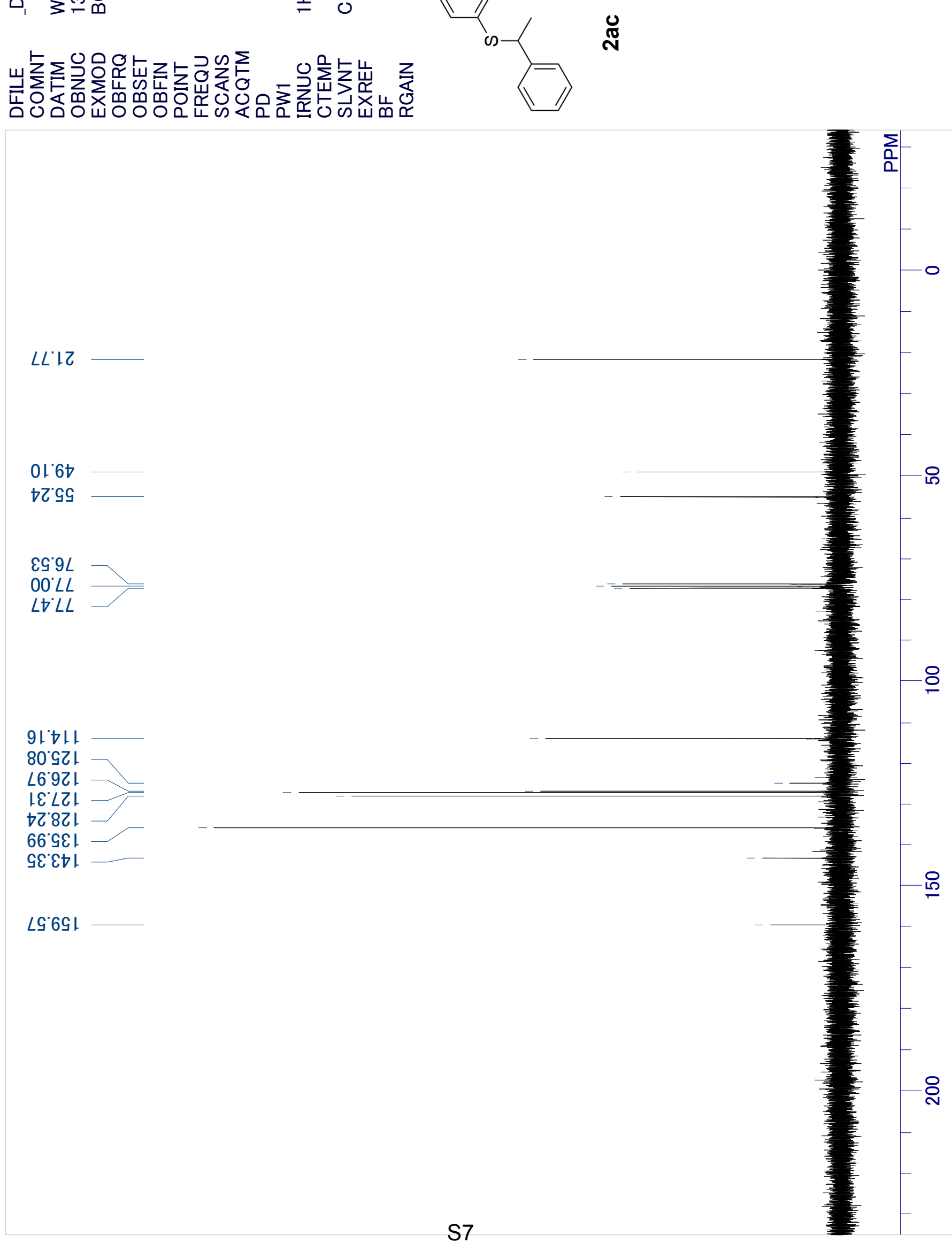


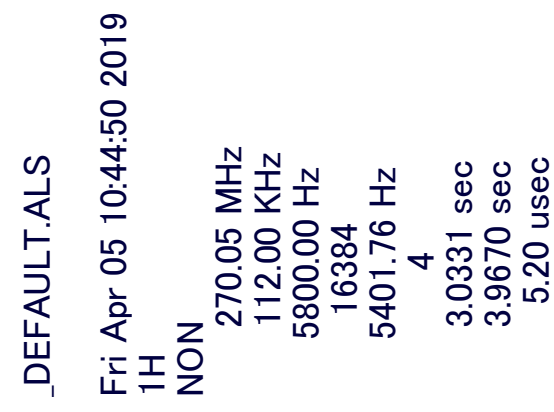

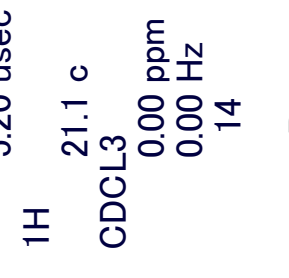

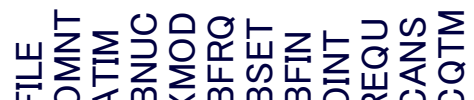

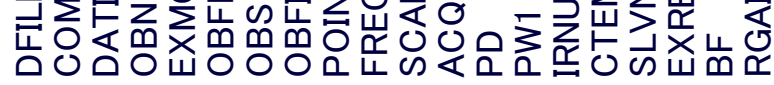

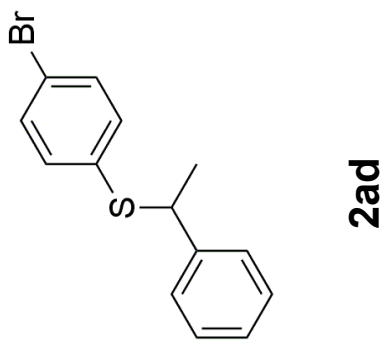
$00^{\circ} 0$

09.1

$29^{\circ} \mathrm{L}$ 


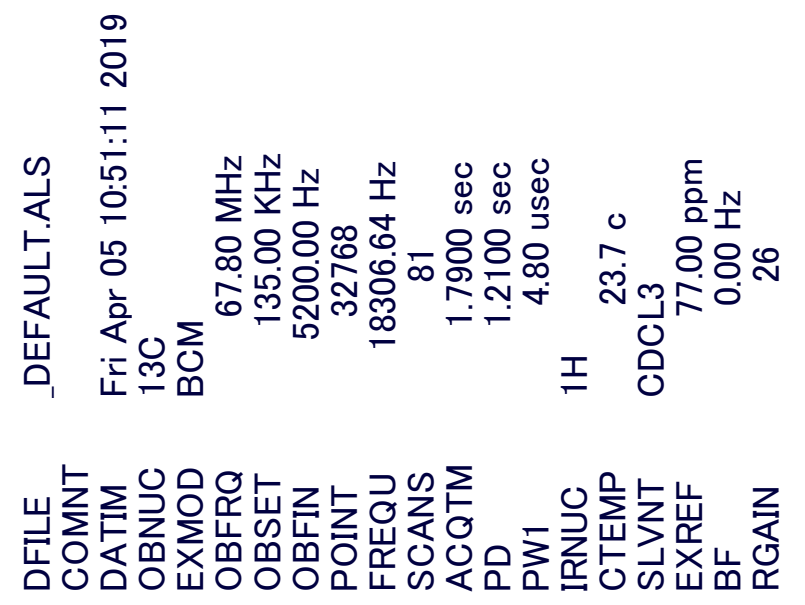

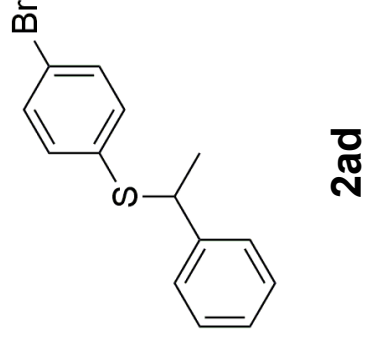

oz'z२

9080

$\varepsilon 9^{9} 9 L$

00 LL

$8 D^{\circ} L L$

$0 \varepsilon^{\circ}|Z|$

IZ LZI

9Z LZ।

\&† $8 Z 1$

$|L| L \mid$

$\angle 6 \varepsilon \varepsilon$ ।

$\angle I " \nabla \varepsilon \mid$

8Lてヤ।

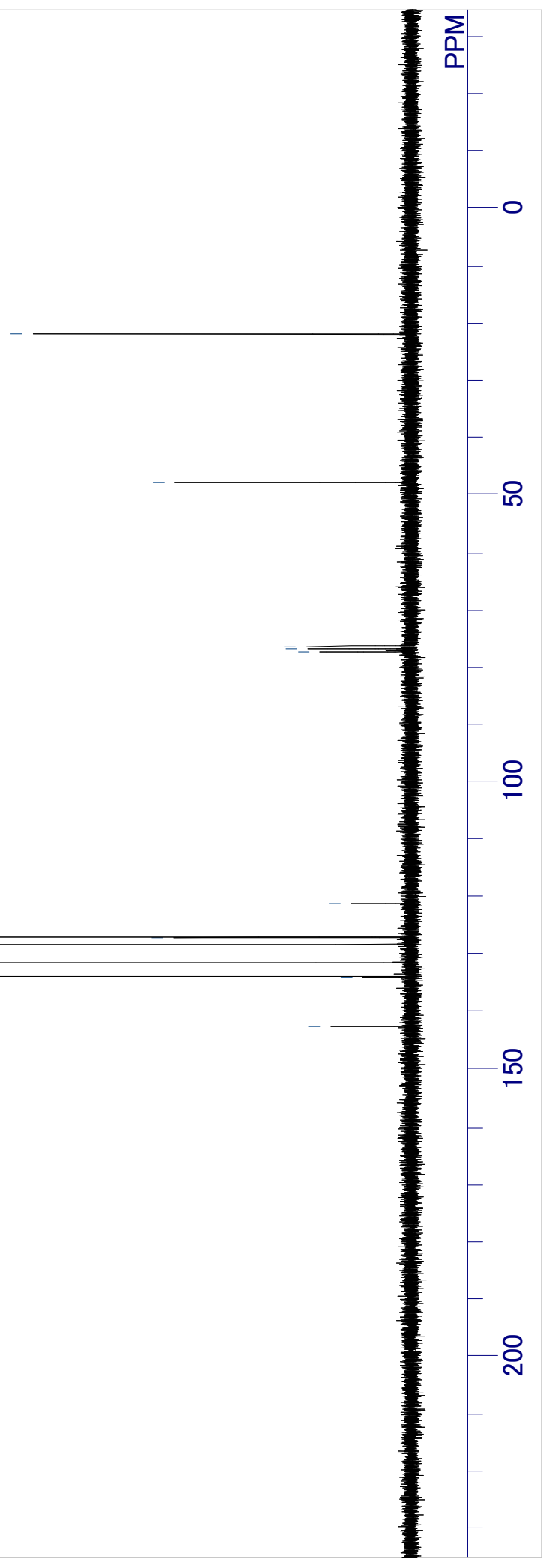




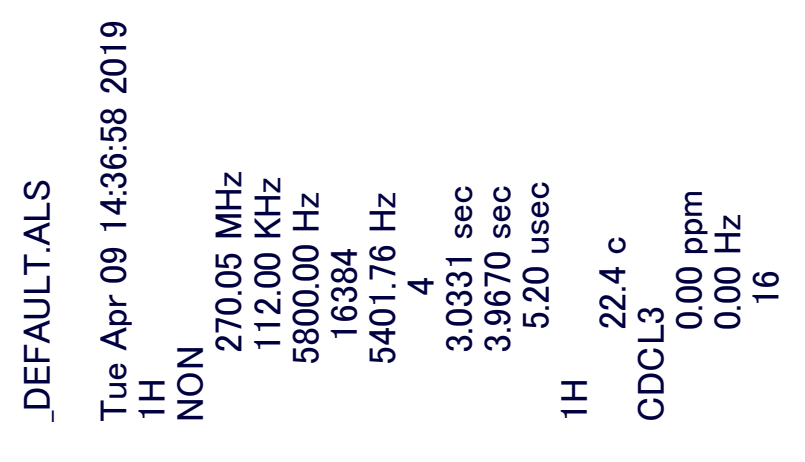

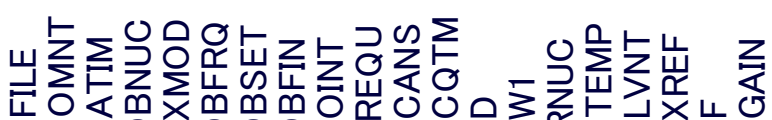

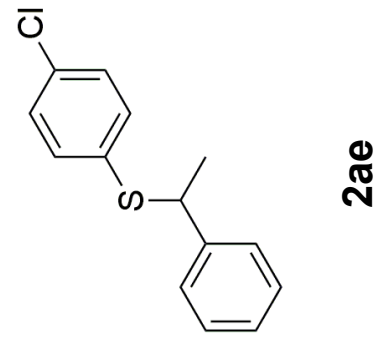

000

$\varepsilon G^{\prime}$

09 .

$\varepsilon 9^{\circ}$ ।

GZt

$8 Z 7$

$0 \varepsilon^{\circ} t$

$\varepsilon \varepsilon^{\circ} \dagger$

$\angle I L$

$Z Z L$

$Z Z L$

$\varepsilon Z Z^{\prime} L$

$G Z L$

$9 Z ' L$

$L Z L$

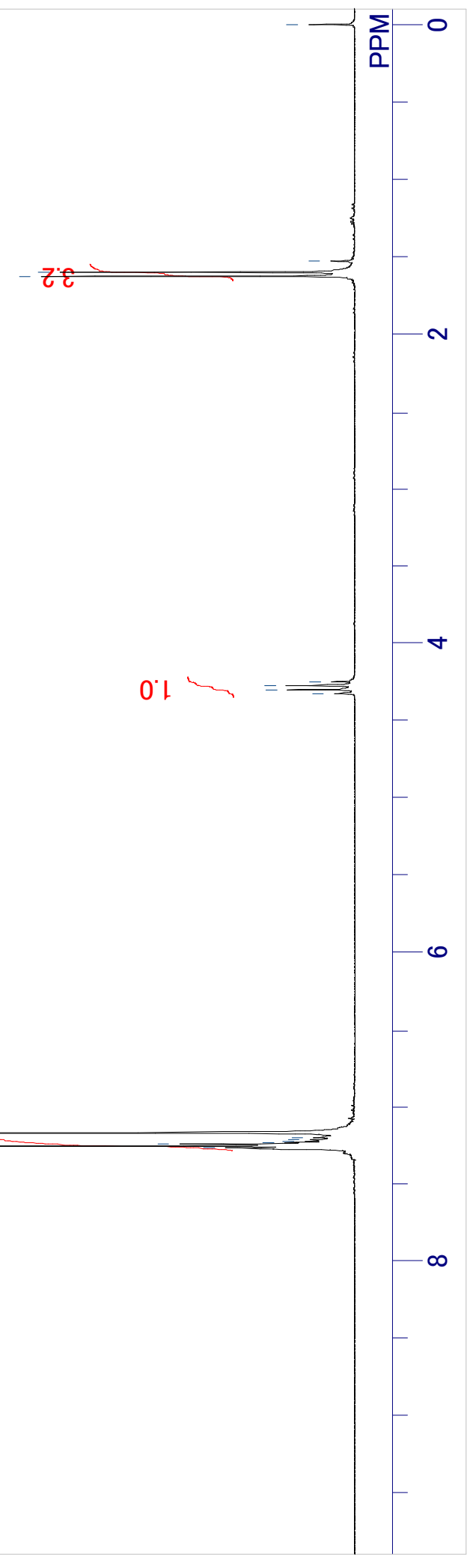




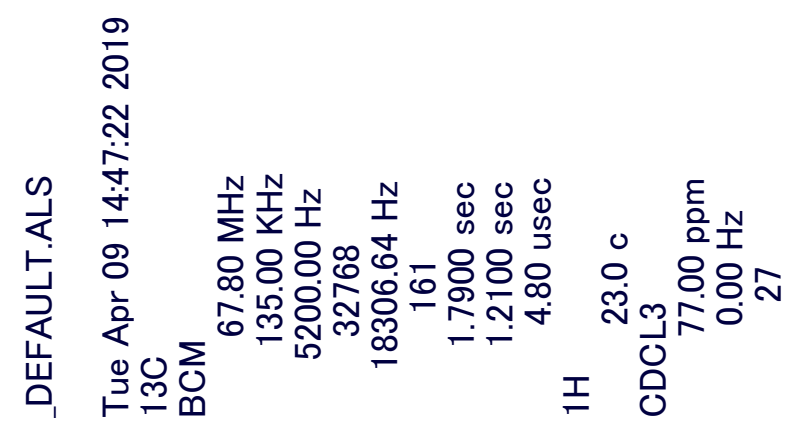

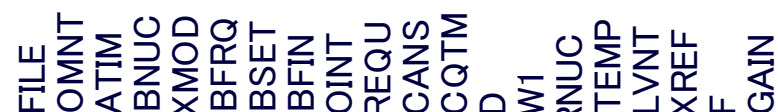

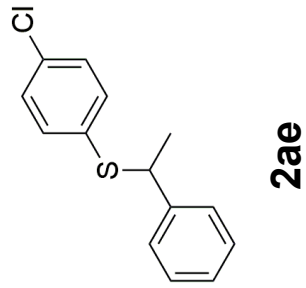

$9 ! Z Z$

9278

$\varepsilon G \cdot 9 L$

$00^{\circ} L L$

$\angle t^{\circ} L L$

$\varepsilon Z\llcorner Z$ L

9Z' LZI

乙† $8 Z 1$

$6 L \cdot 8 Z 1$

$\varepsilon \succ \varepsilon \varepsilon \mid$

乙6. $\varepsilon$ เ

て8てヤト 


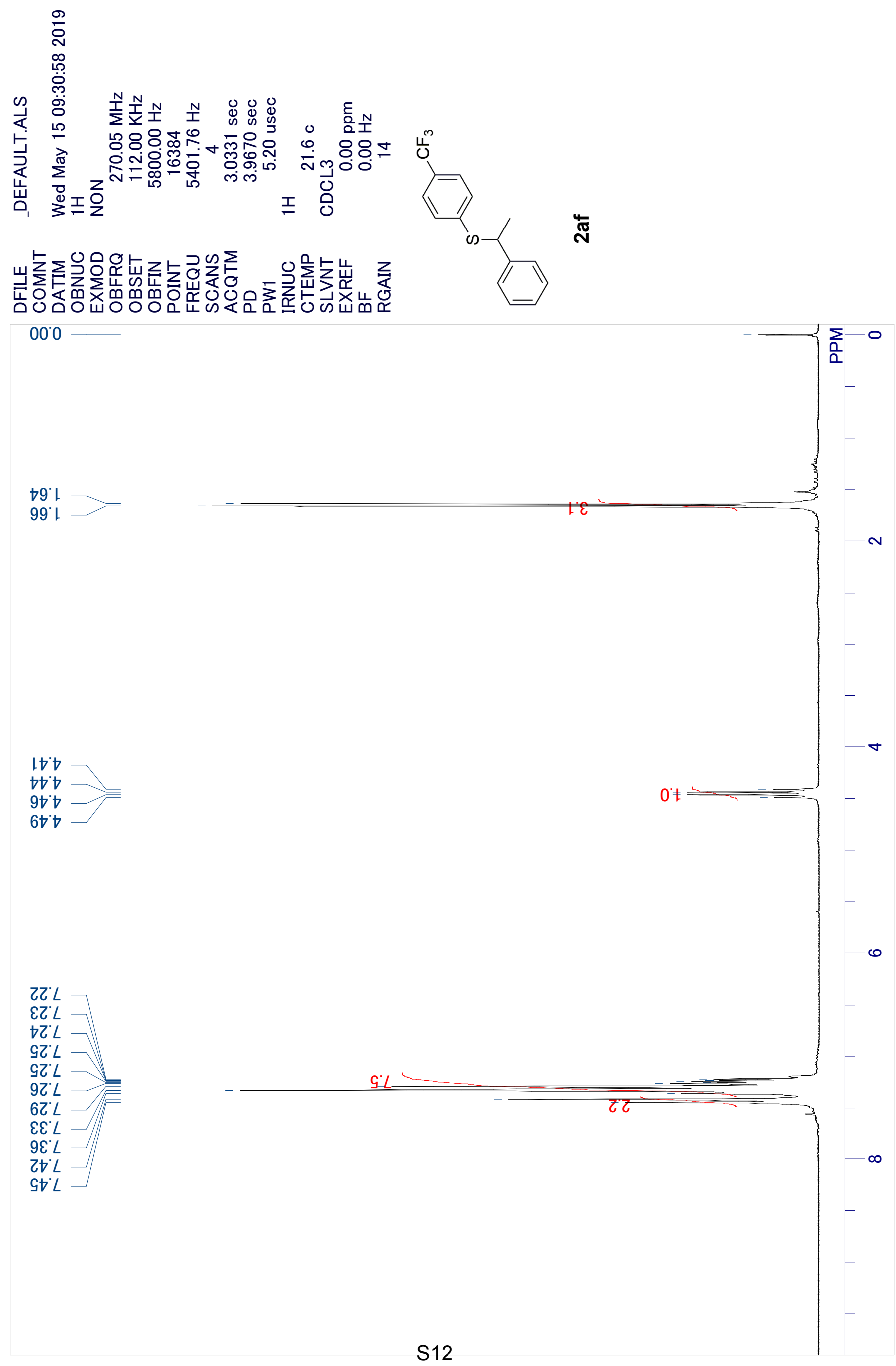




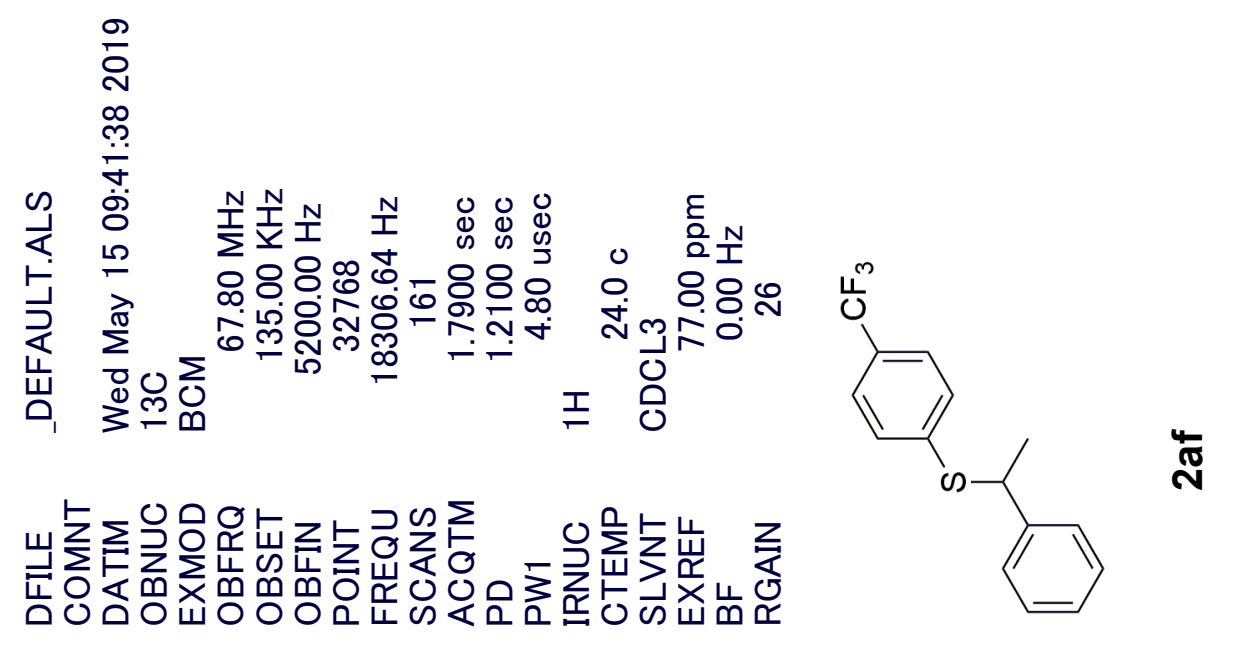

1927

$\nabla 0<\nabla$

EG'9L

$00^{\circ} L L$

$87^{\circ} L L$

$\angle 0 \mathrm{ZZ}$

9ह'G乙।

ItGて।

L७G乙L

EG G乙L

GILZL

Gt LZL

098Z1

$\angle 9{ }^{\circ} 8 \mathrm{~L}$

0 $0 \varepsilon^{\circ} 0 \varepsilon$

06.0t L

9G'Zt। 


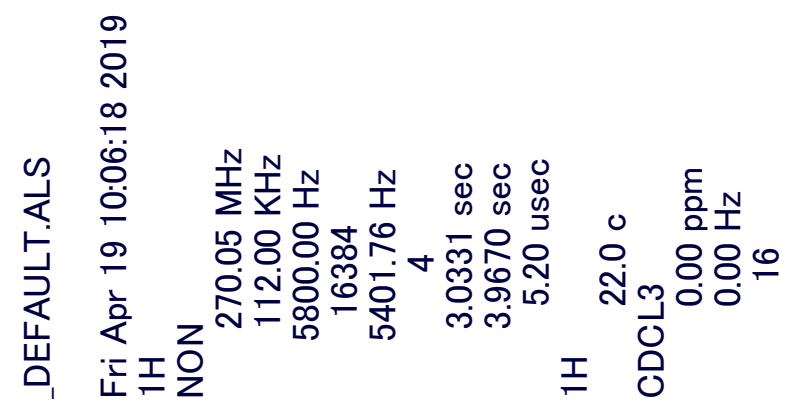

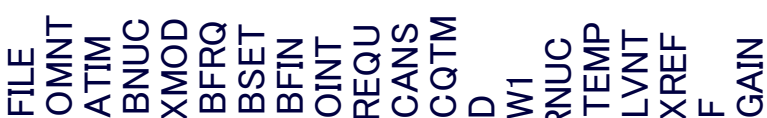

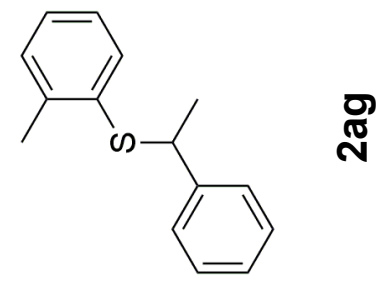
$00^{\circ} 0$

$29^{\circ}$

99.

乙๕乙

G't

$87 t$

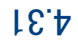

$\varepsilon \varepsilon^{\circ}$

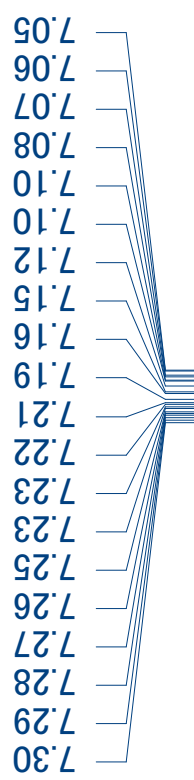

001

0.1 


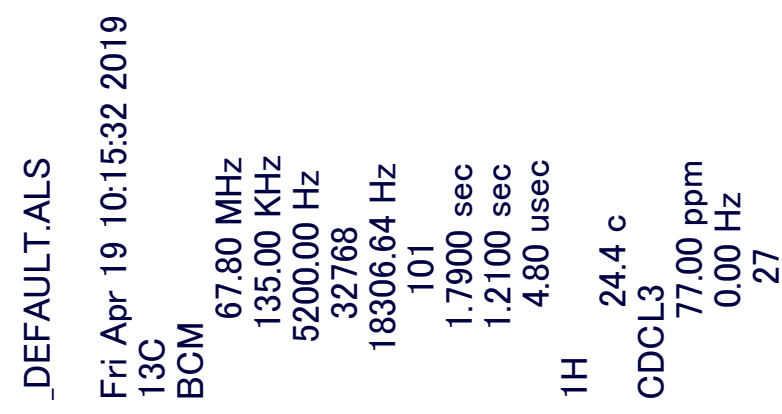

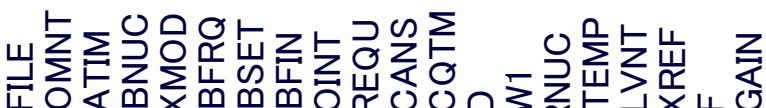
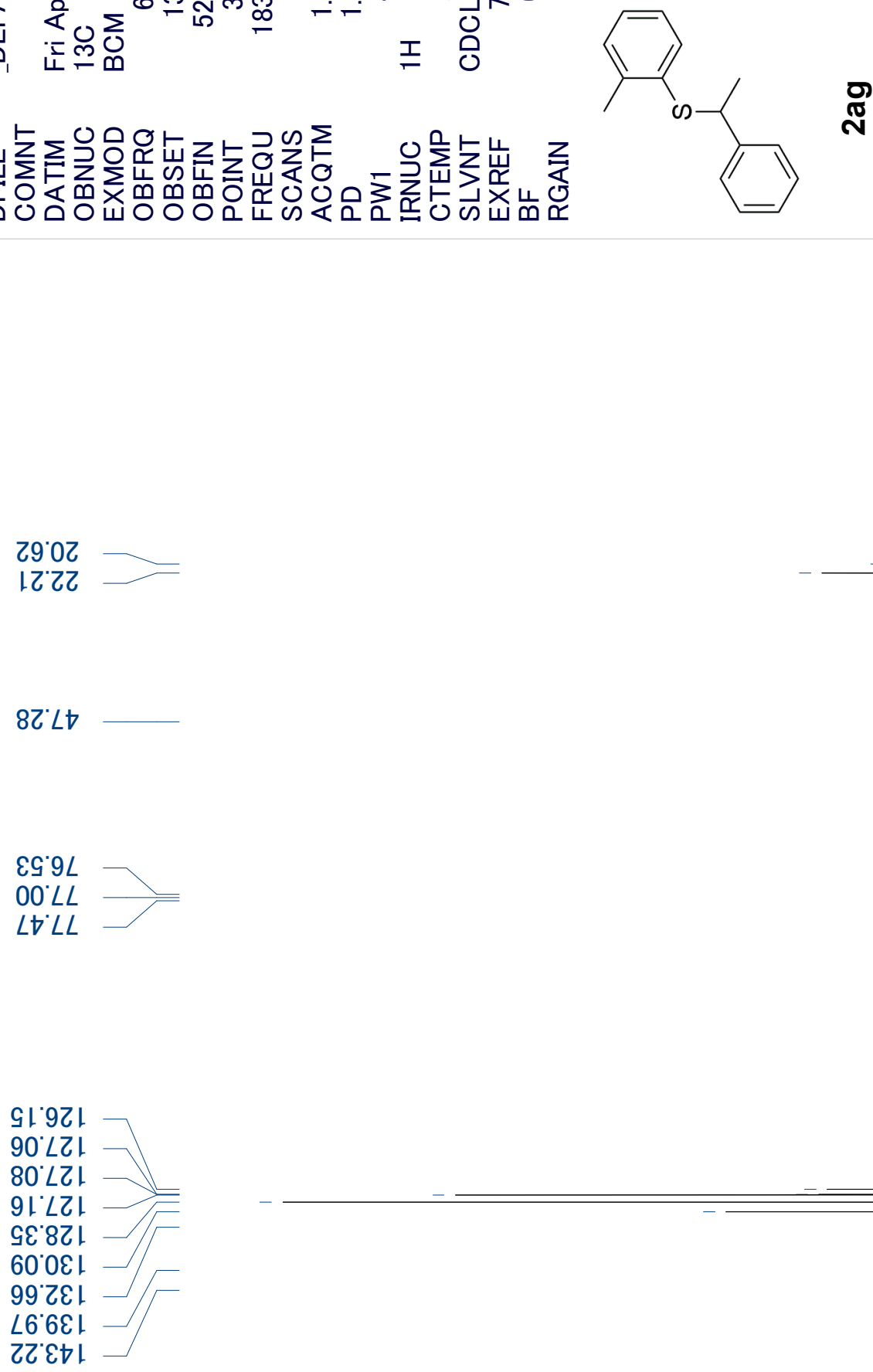


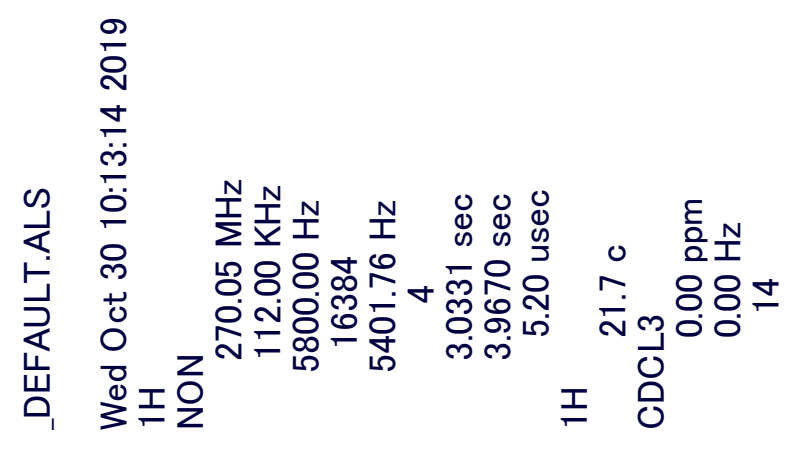

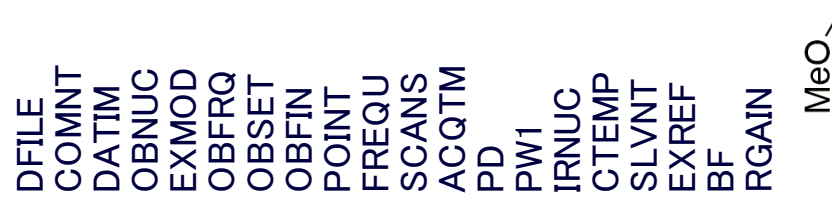

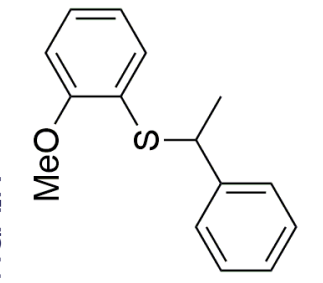

$\frac{c}{N}$ $00^{\circ} 0$

$09^{\circ} 1$

\&9.
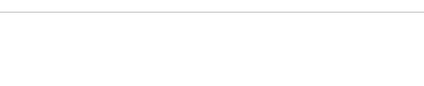

${ }^{\circ} \mathcal{\varepsilon}$

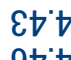

9t

0.1

IS'

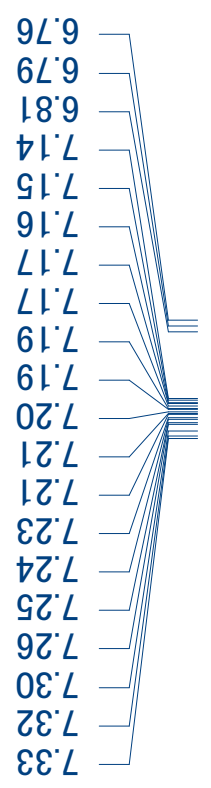




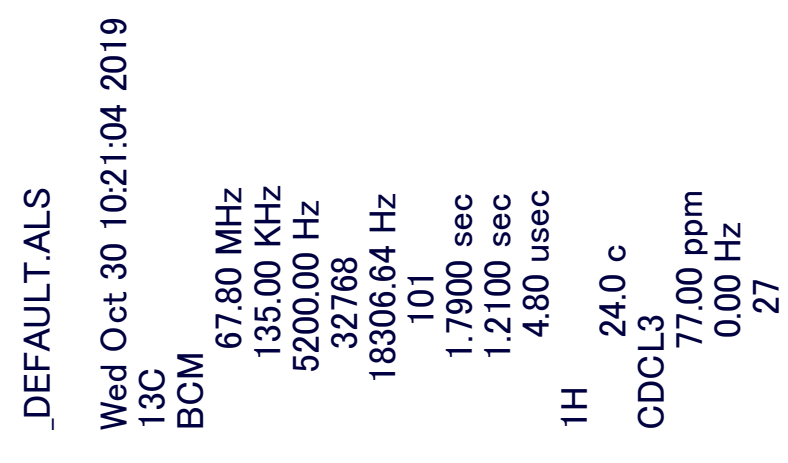

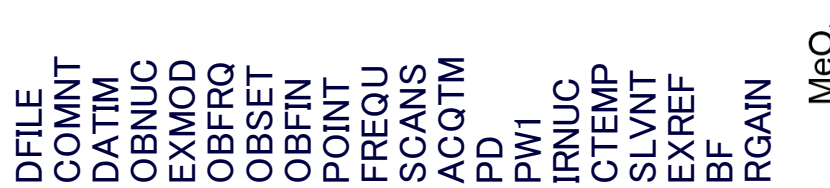

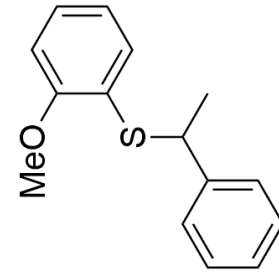

$\frac{E}{N}$

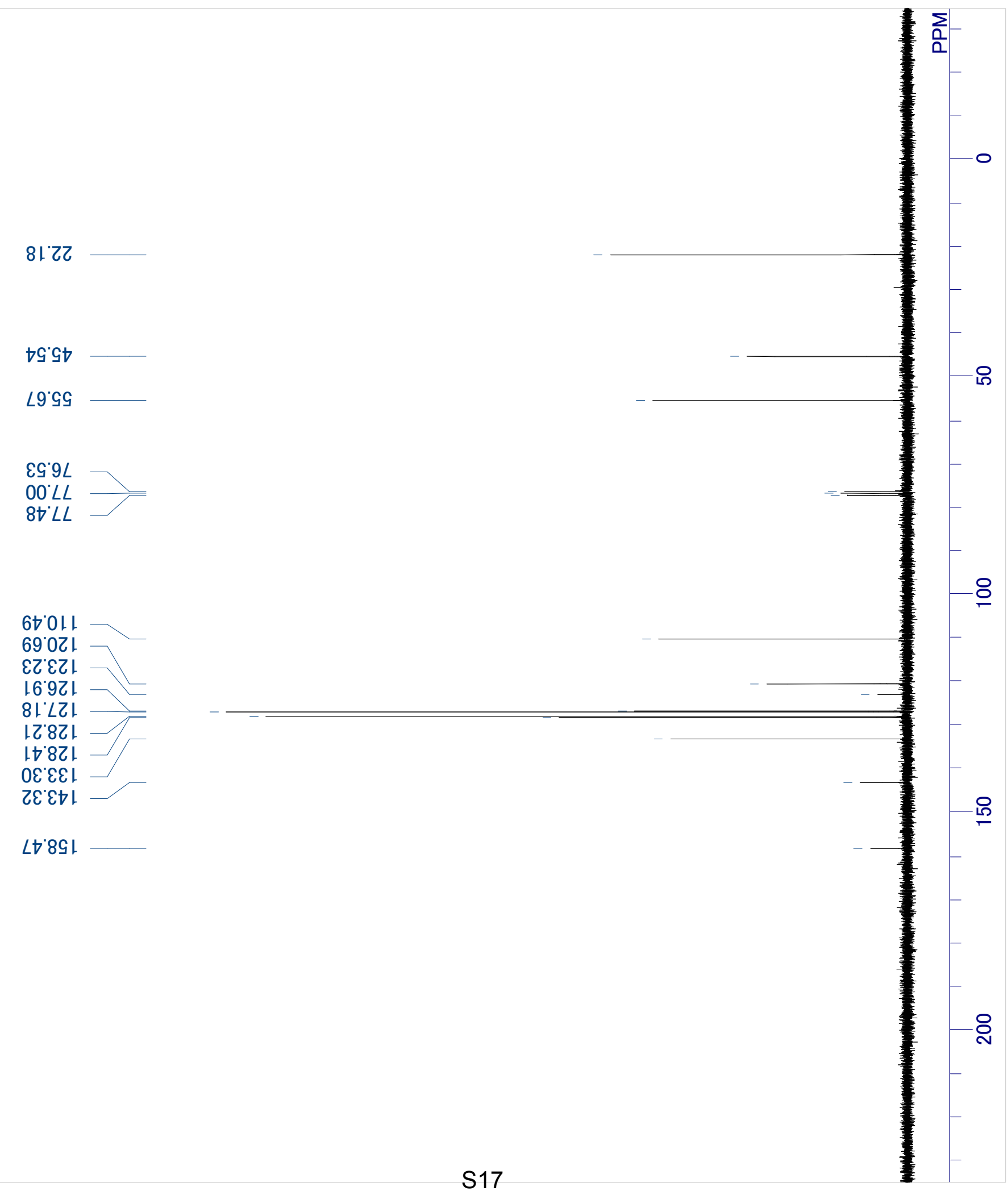




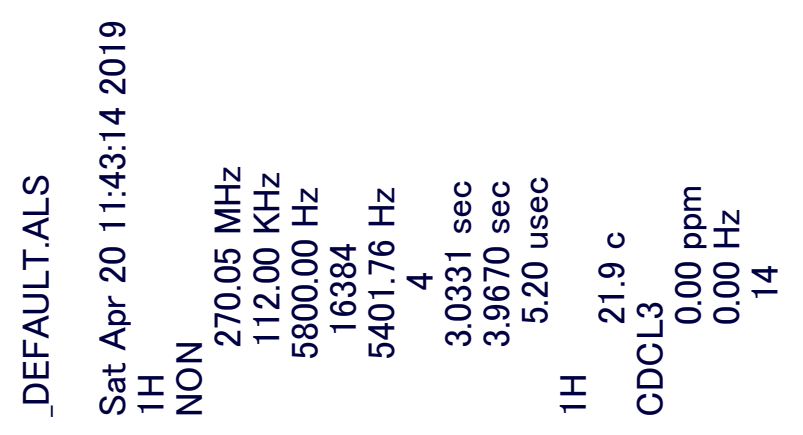

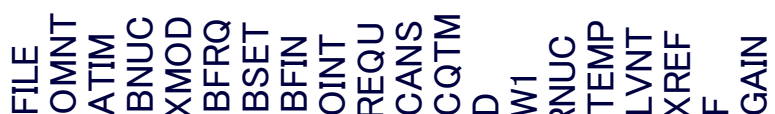

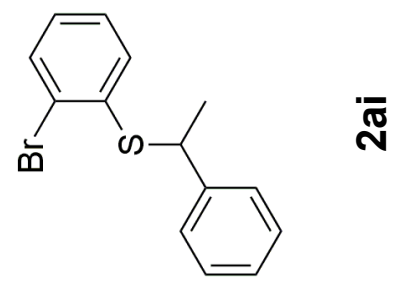
$00^{\circ} 0$

$79^{\circ}$

$\angle 9^{\circ}$ L
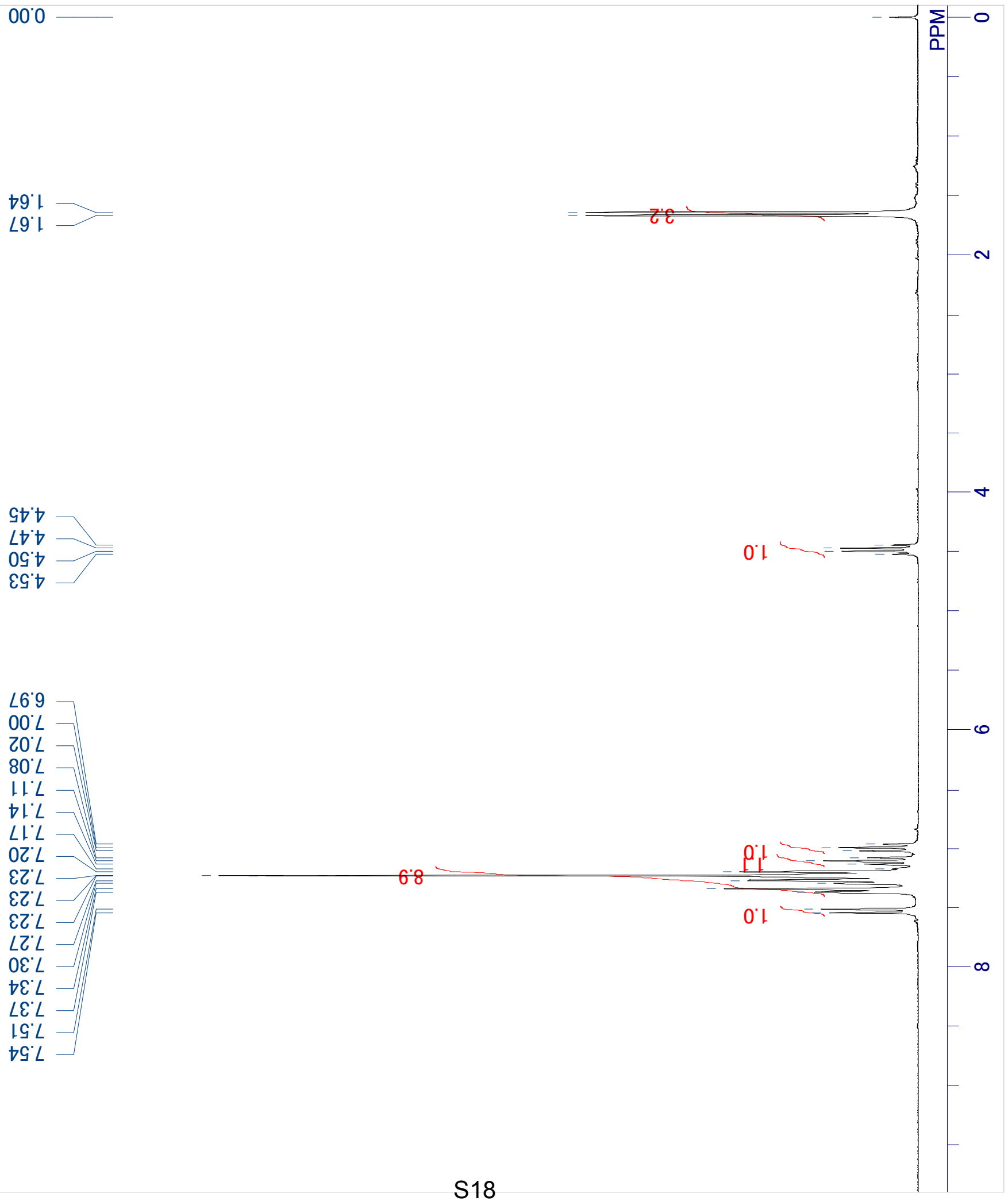


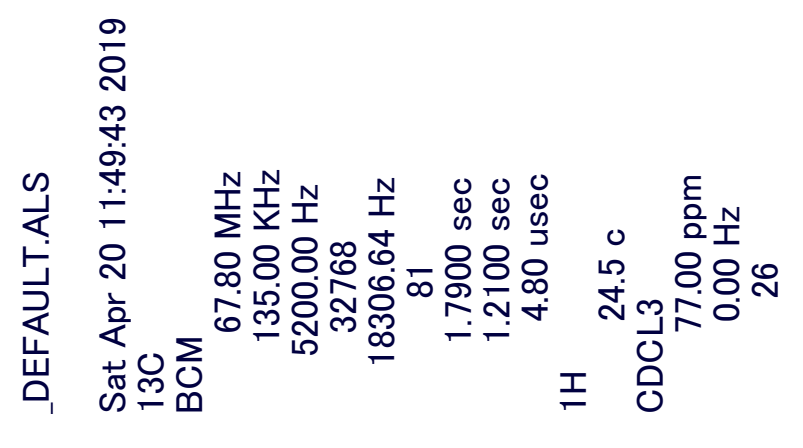

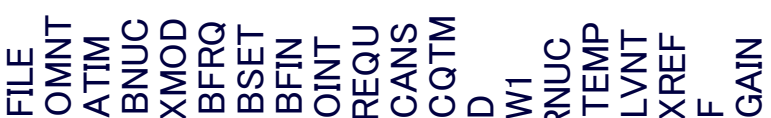
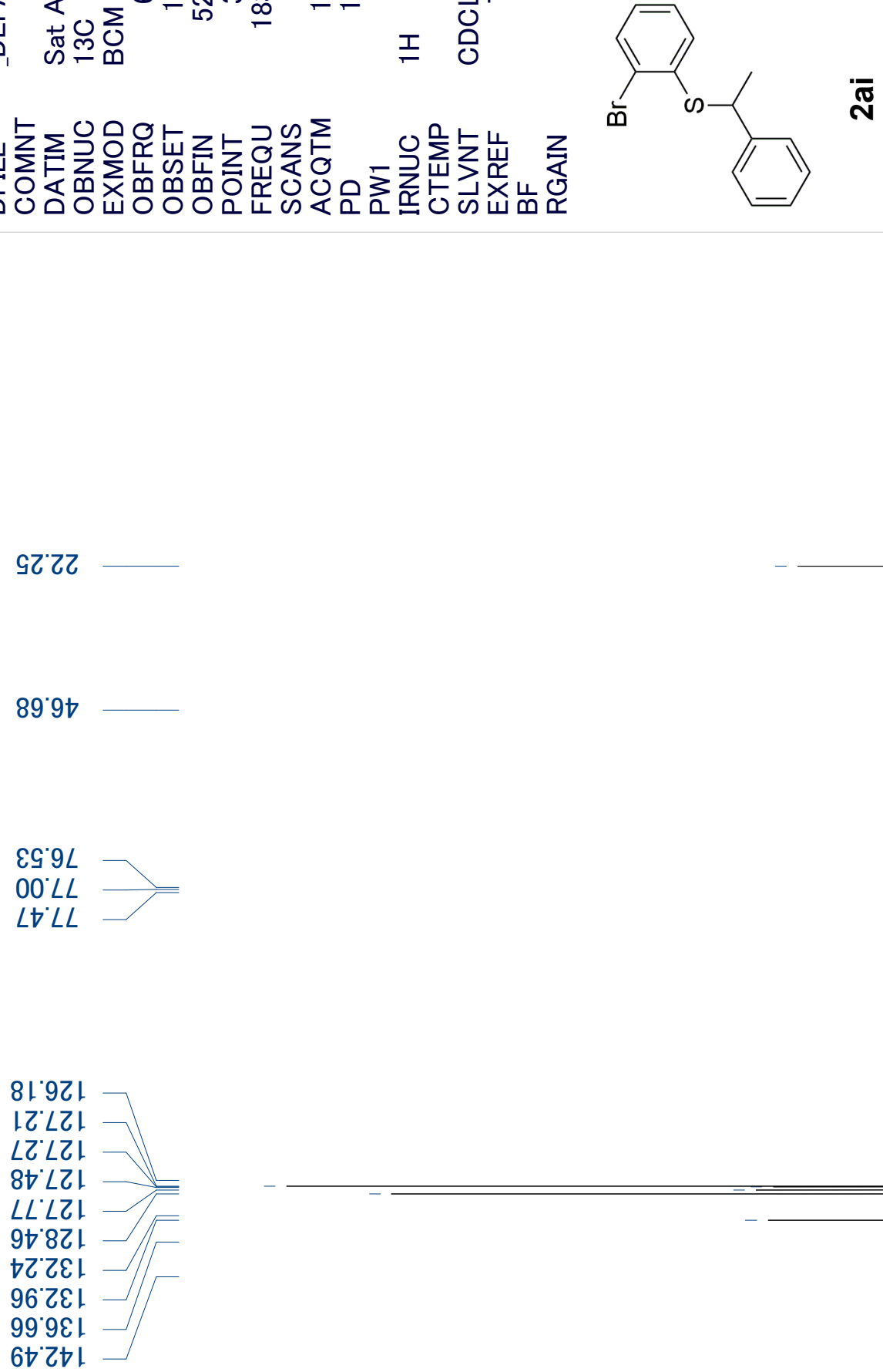


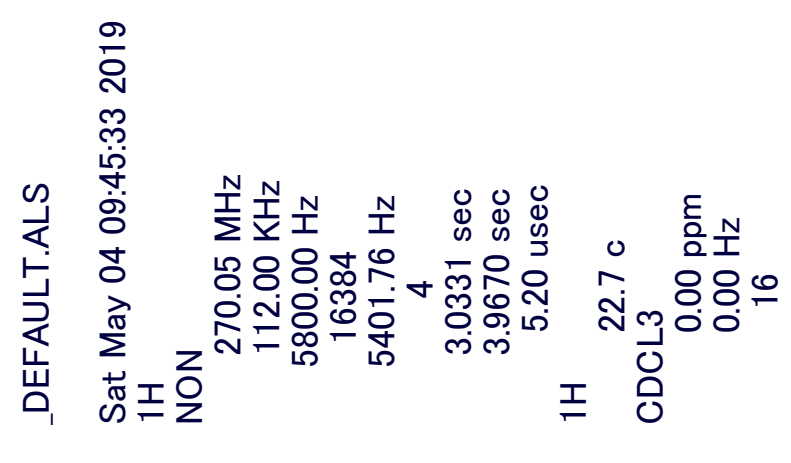

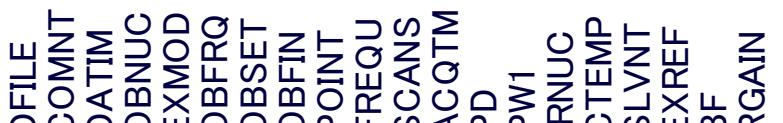

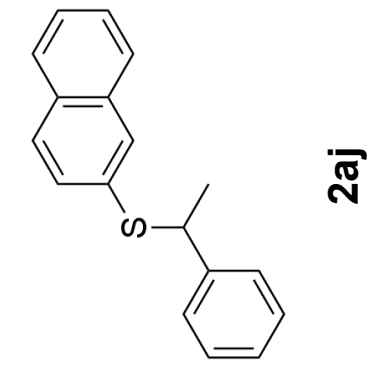

$$
000
$$

IS'

s9.

$\angle 9^{\circ}$ ।

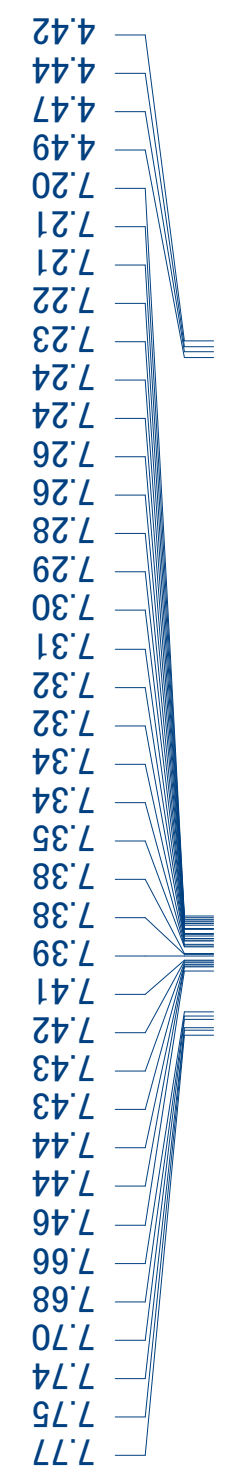

$\varepsilon \cdot 6$

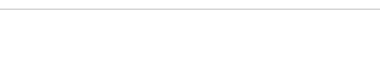



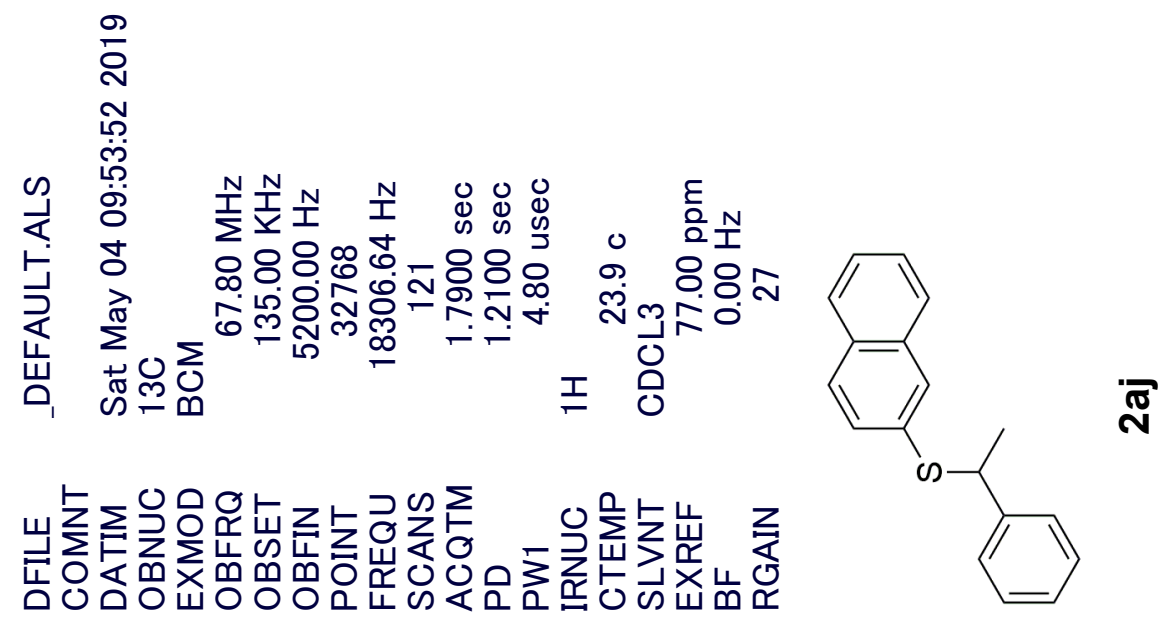

$\downarrow \varepsilon \vee 乙 乙$

$\angle 8^{\circ} L t$

ZG'9L

$00^{\circ} L L$

$\angle \nabla^{\circ} L L$

66.9乙।

6乙9亿।

$\angle L L Z L$

$\angle Z L Z L$

$\angle \varepsilon^{\prime} L Z L$

6G'LZL

이이

乙† 8Z

$\varepsilon L ' 6 Z$ L

EO IEL

乙乙乙E

6马'ZE

EG. $\varepsilon \mathcal{~ L ~}$

이라 


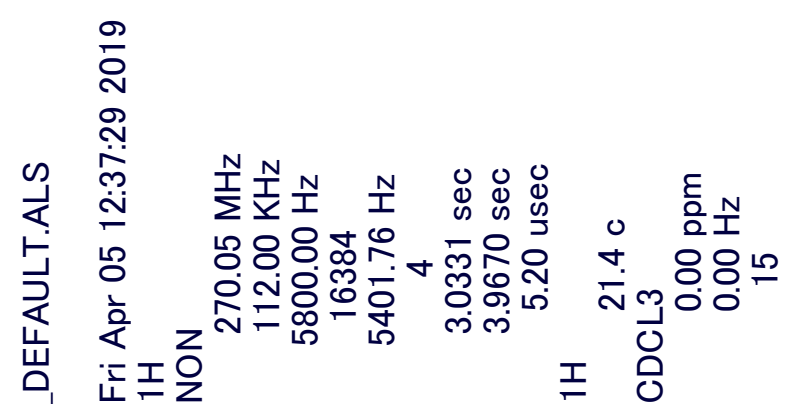

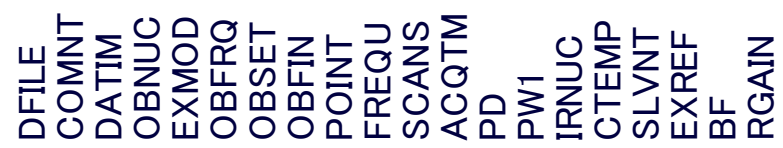
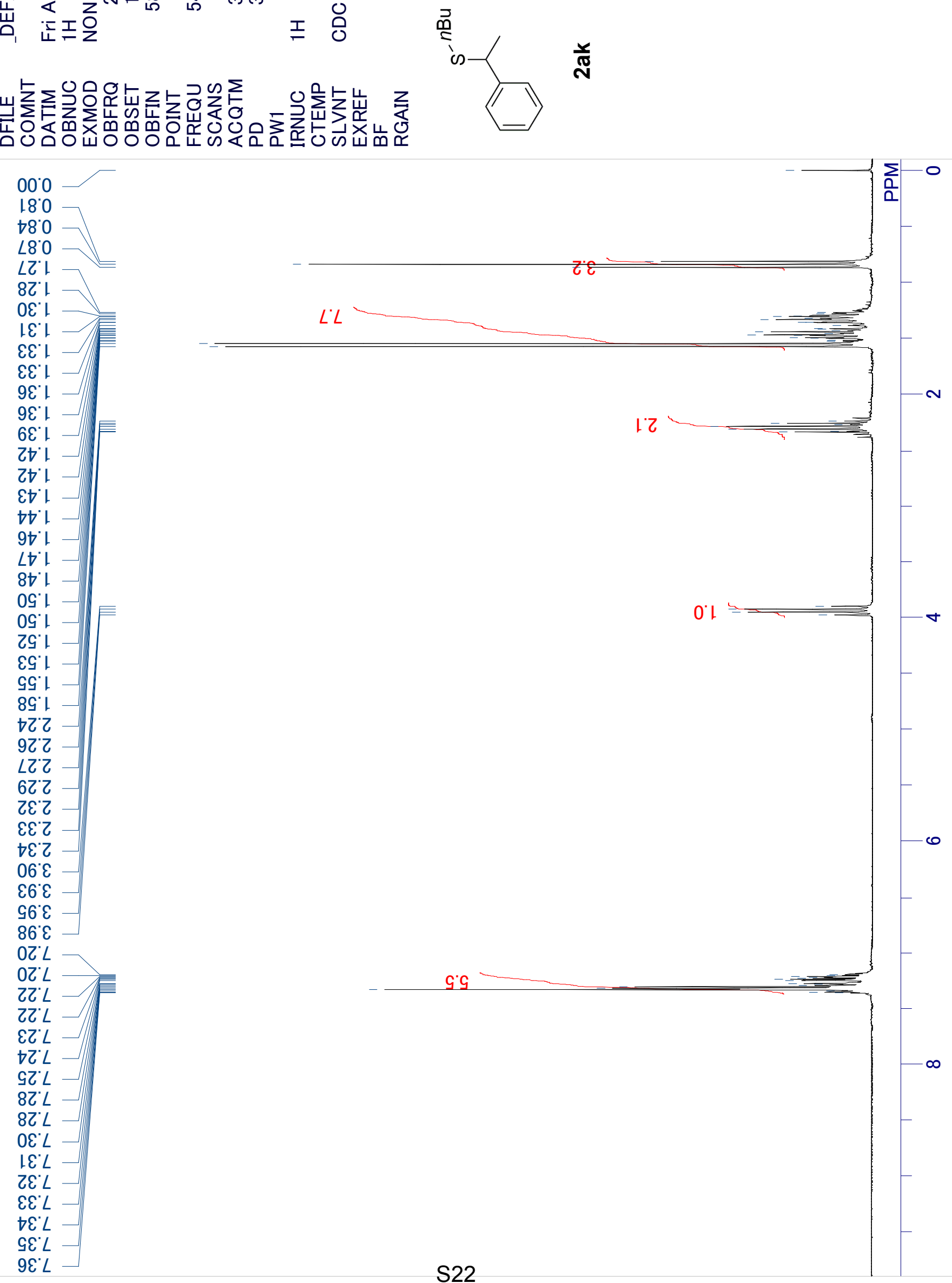


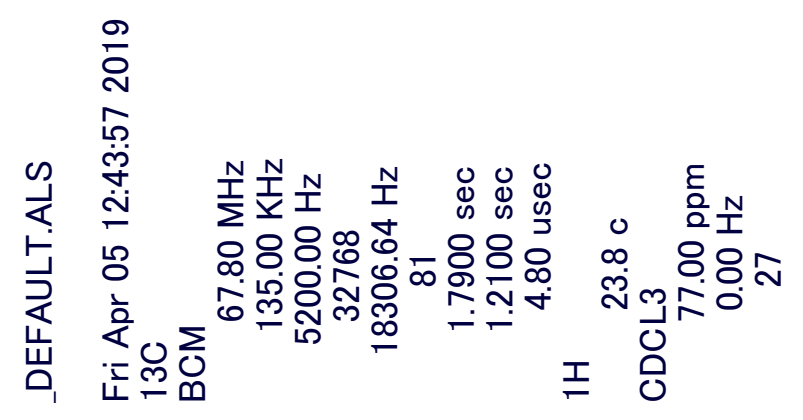

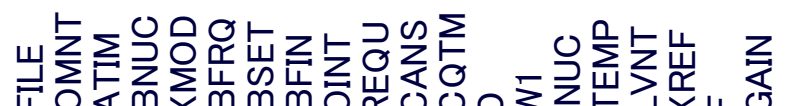

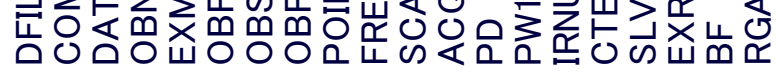

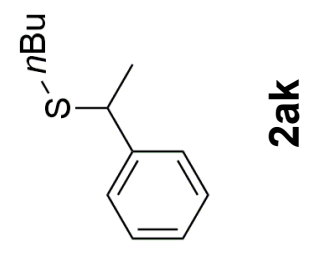

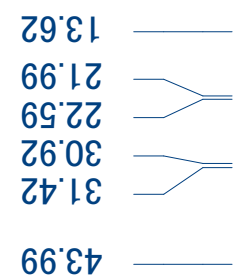

$66^{\circ} \varepsilon \supset$

\&G. $9 L$

00 LL

$\angle t L L$

68.9Z1

OZ LZ।

$\angle \varepsilon^{\circ} 8 Z$ L

0でカtト 


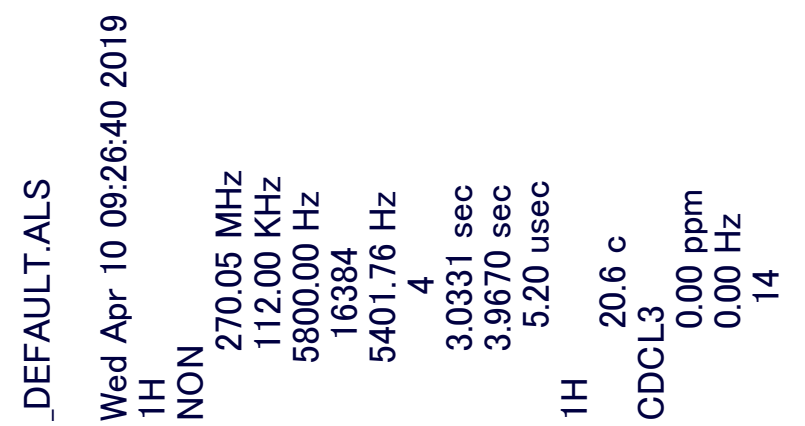

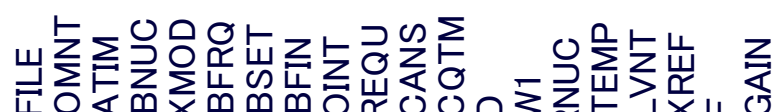

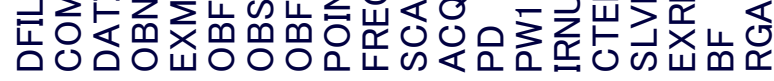

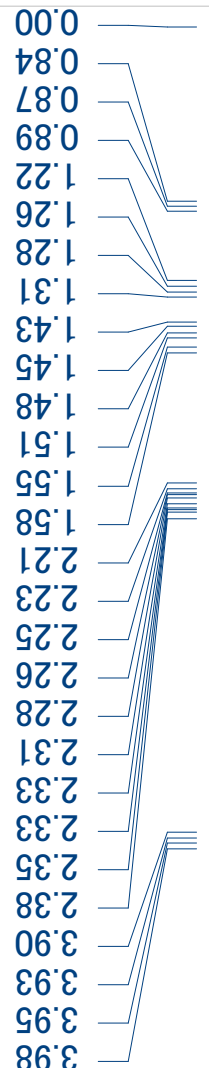<smiles>C=[Hg]C</smiles><smiles>C1COCO1</smiles>

\section{$\bar{\Xi}$}
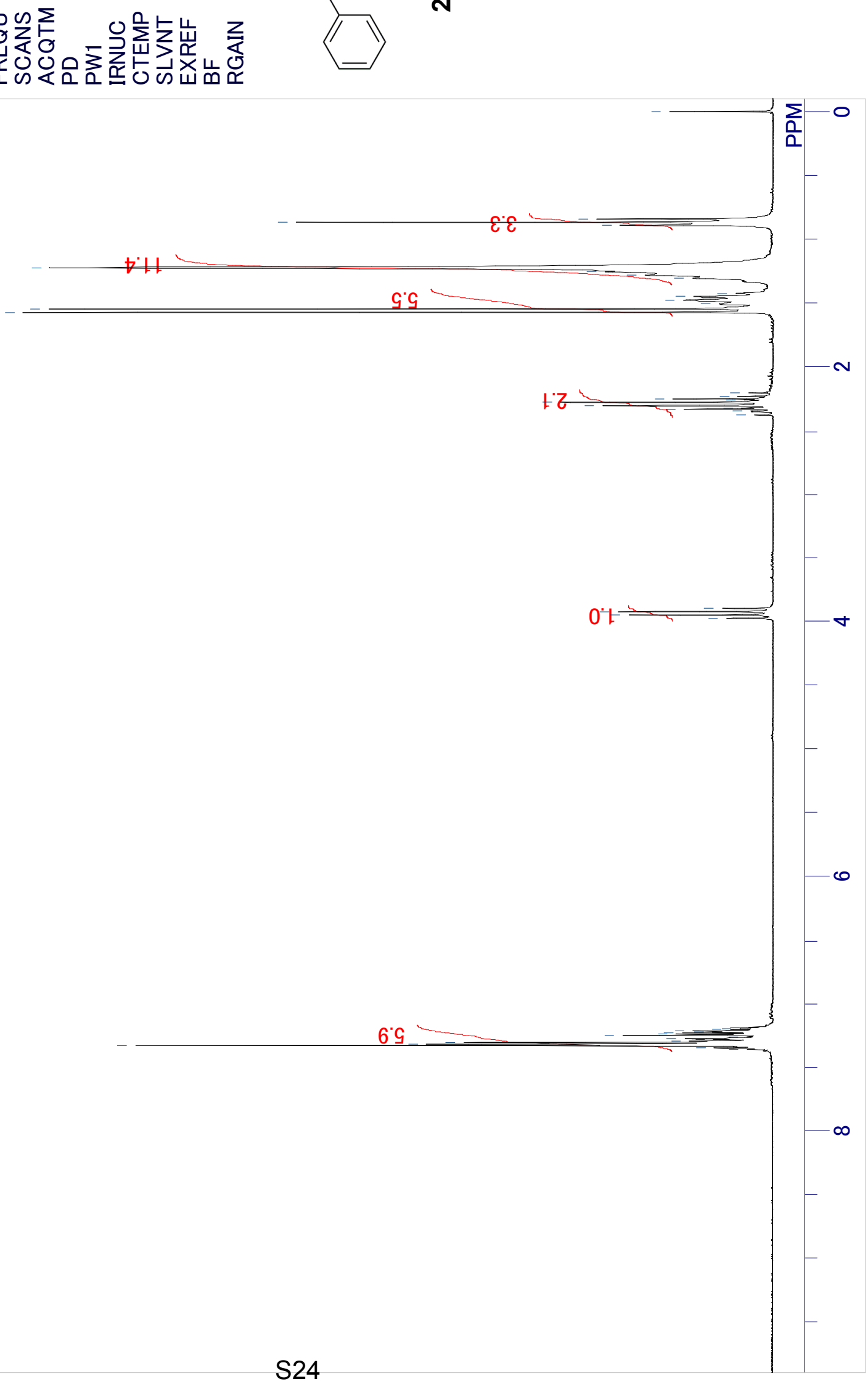


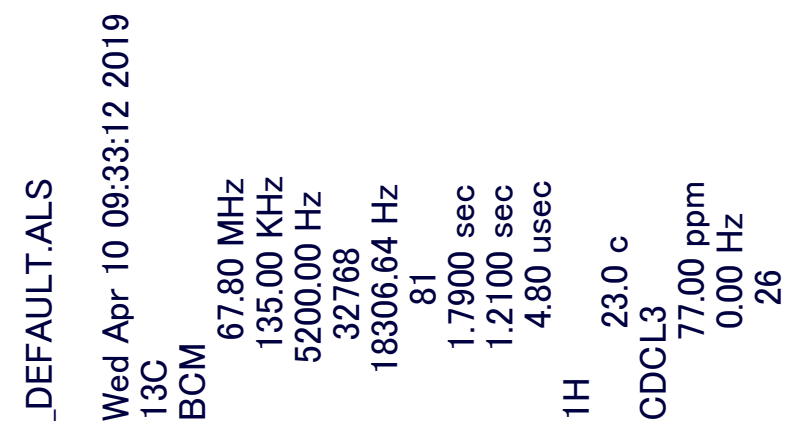

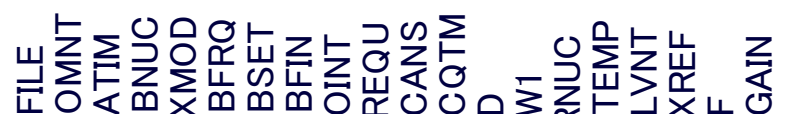
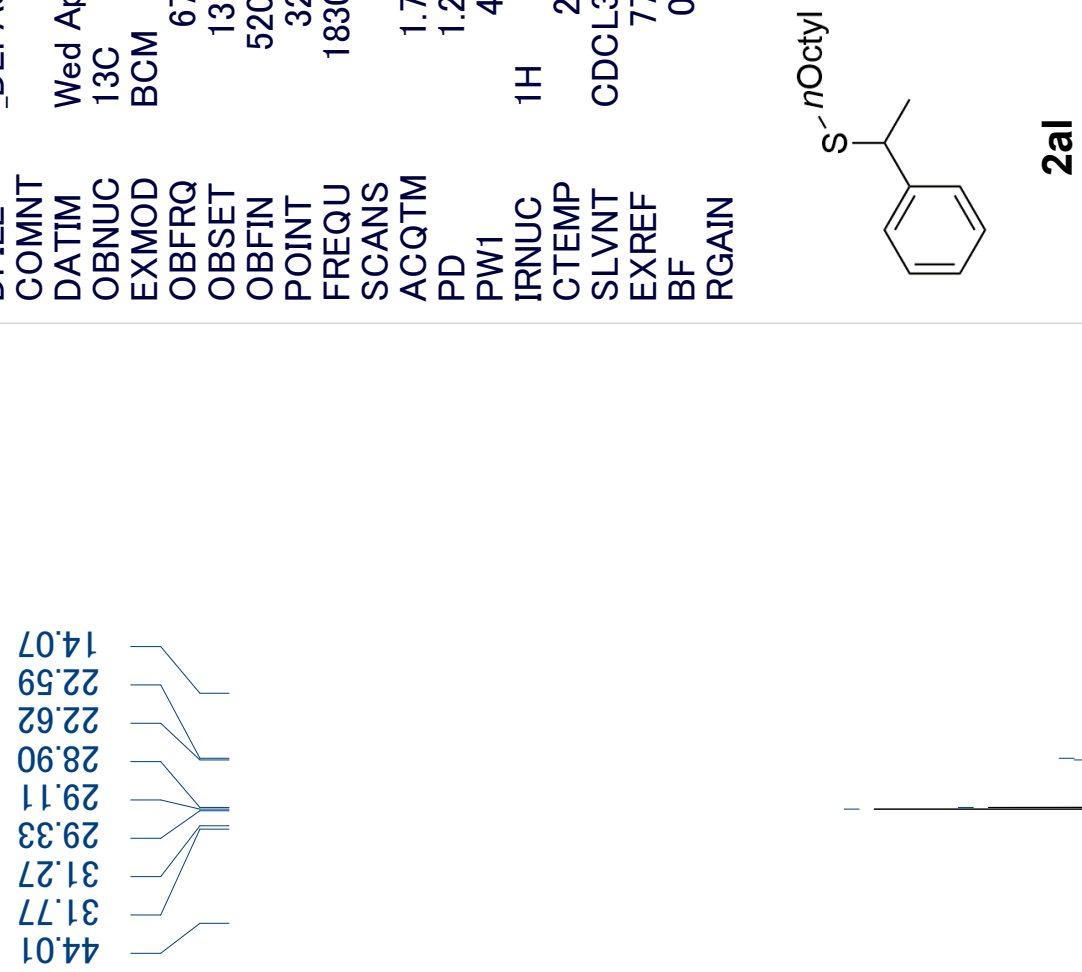

ZG. $9 L$

$00 \angle L$

$\angle t L L$

68.9Z।

$0 Z$ LZI

$\angle \mathcal{E} 8 Z$ ।

レでャト 


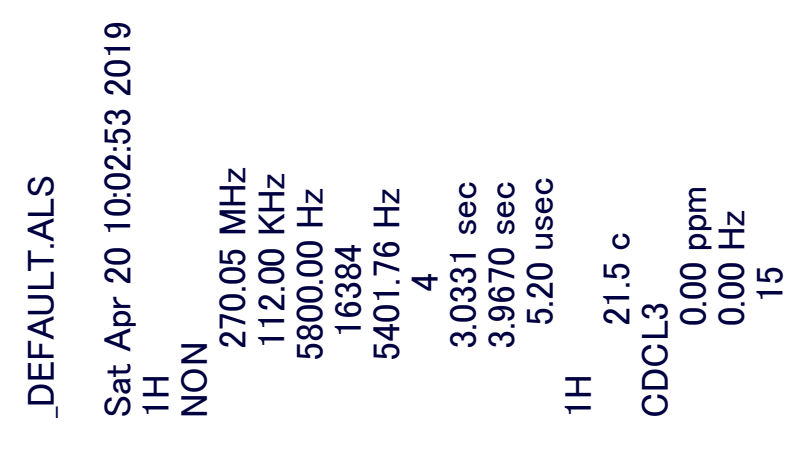

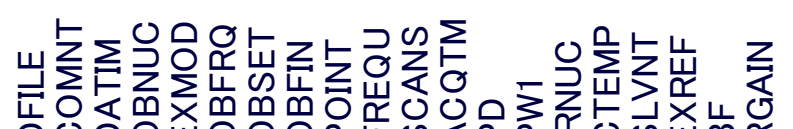

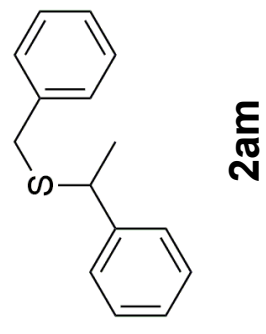

$00^{\circ} 0$

IG':

$t \mathcal{C}^{\prime}+\longrightarrow$

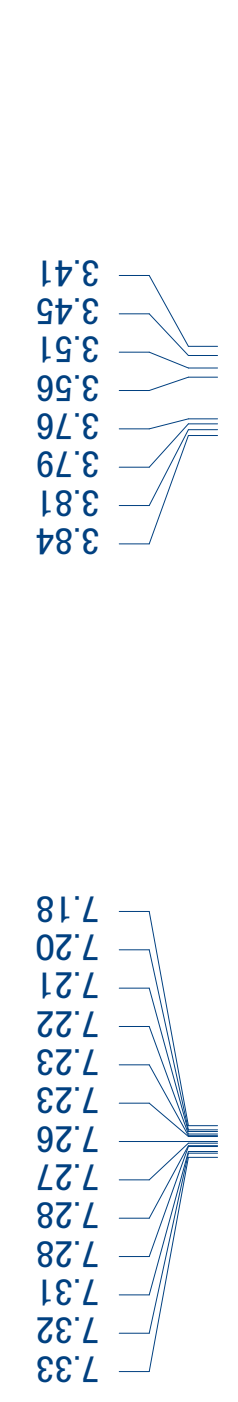

ลี

12

0. 1 


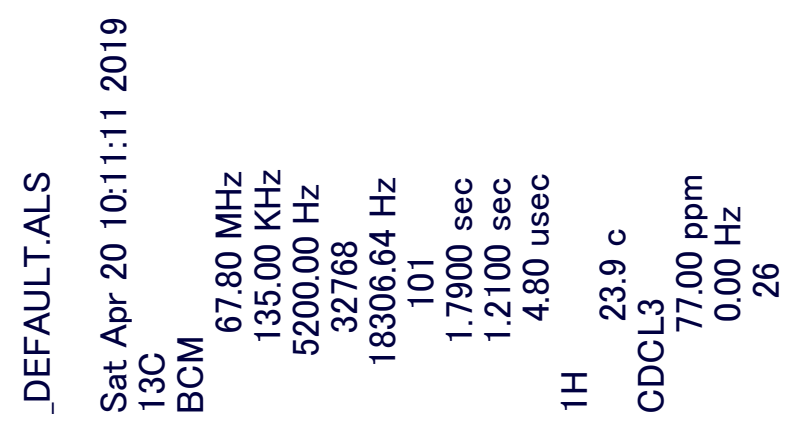

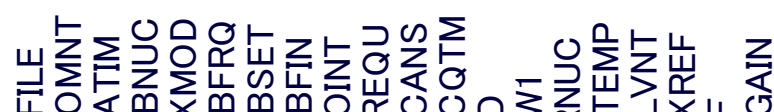<smiles>CC(C)OCc1ccccc1</smiles>

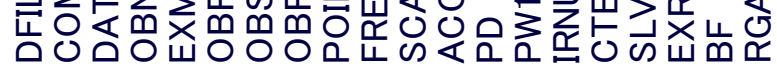

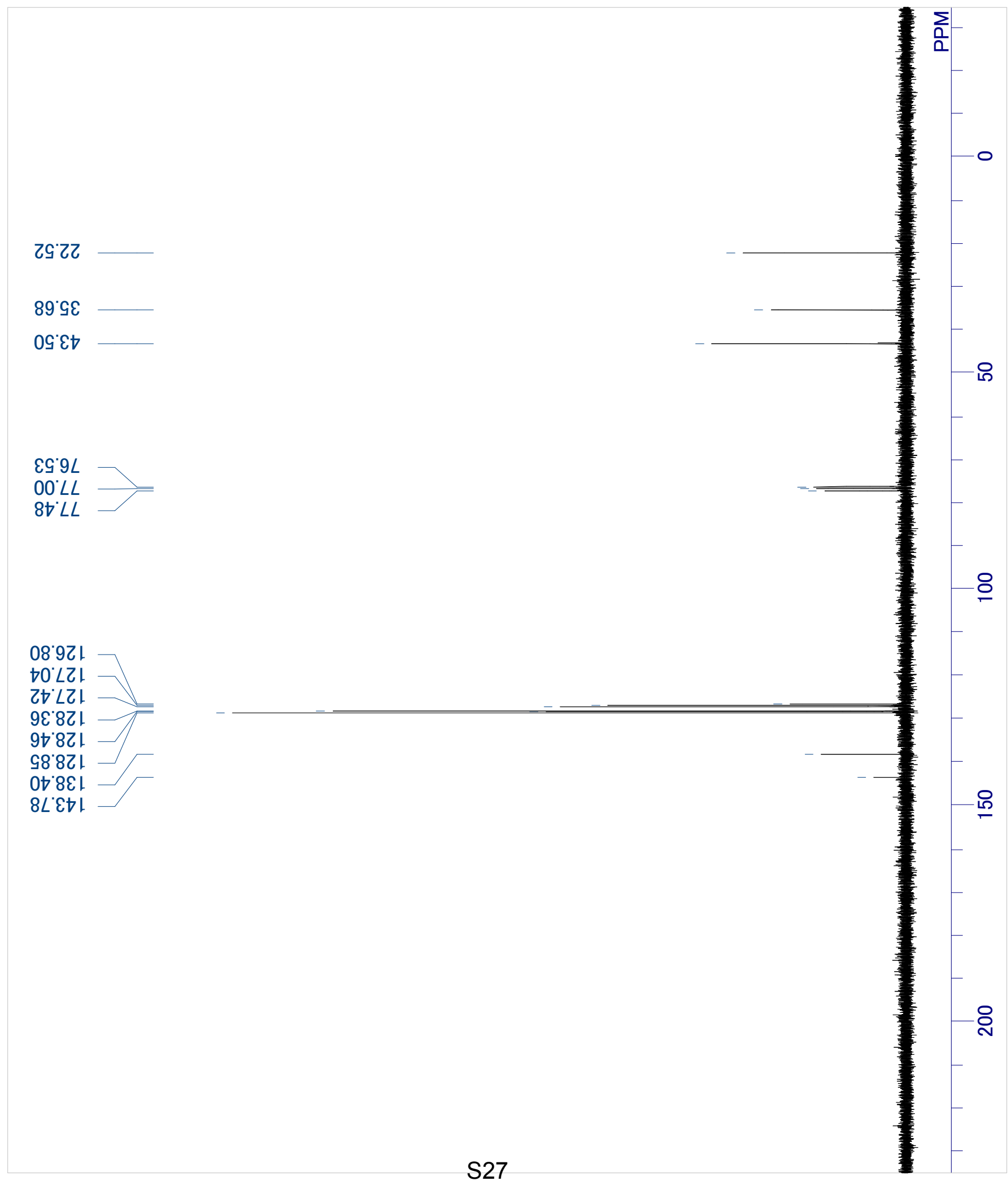




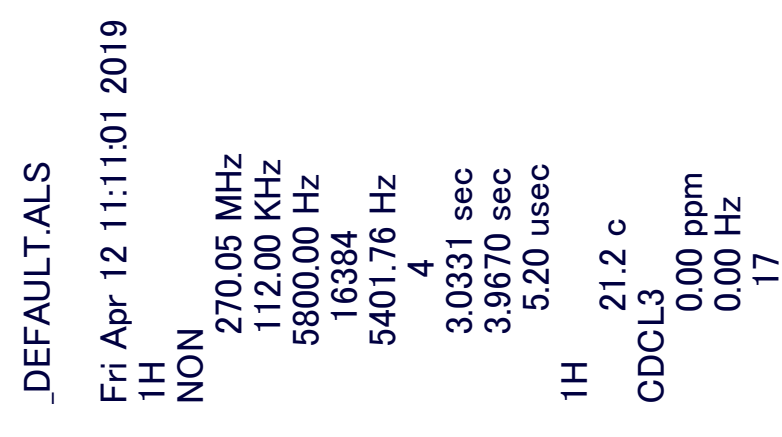

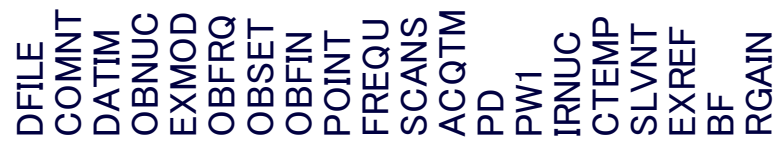

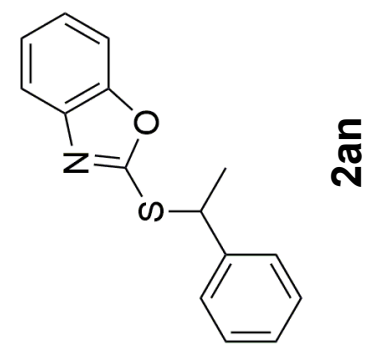

$00^{\circ} 0$

$\varepsilon 9^{\circ}$

$88^{\circ}$.

$06^{\circ} \mathrm{L}$

019

El'

GI'

819

$0 Z$ L

$0 Z$ L

$\varepsilon Z^{\prime} L$

$\varepsilon Z^{\prime} L$

GZ' $L$

$G Z ' L$

$9 Z^{\prime} L$

$\angle Z ' L$

$8 Z^{\circ} L$

$8 Z^{\circ} L$

$6 Z^{\circ} L$

$1 \mathcal{E}^{\circ} L$

IE'L

乙E' $L$

ZE'L

$\neg \varepsilon^{\circ} L$

$G \varepsilon^{\circ} L$

$9 \varepsilon^{\circ} L$

$\angle \varepsilon^{\prime} L$

$8 \varepsilon^{\circ} L$

$I \nabla^{\circ} L$

IVL

$\varepsilon \nabla^{\circ} L$

$\nabla \nabla^{\circ} L$

$\nabla \nabla^{\circ} L$

$G t^{\circ} L$

$9 t^{\circ} L$

$9 t^{\circ} L$

$6 t^{\circ} L$

$0 G^{\circ} L$

$09^{\circ} L$

$19^{\circ} \mathrm{L}$

$\varepsilon 9^{\circ} L$

t9 $L$

0.9

L $\mathcal{E}$

$0 \% 1$

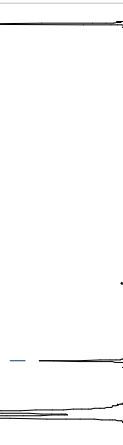

$\sum_{0} 0$ 


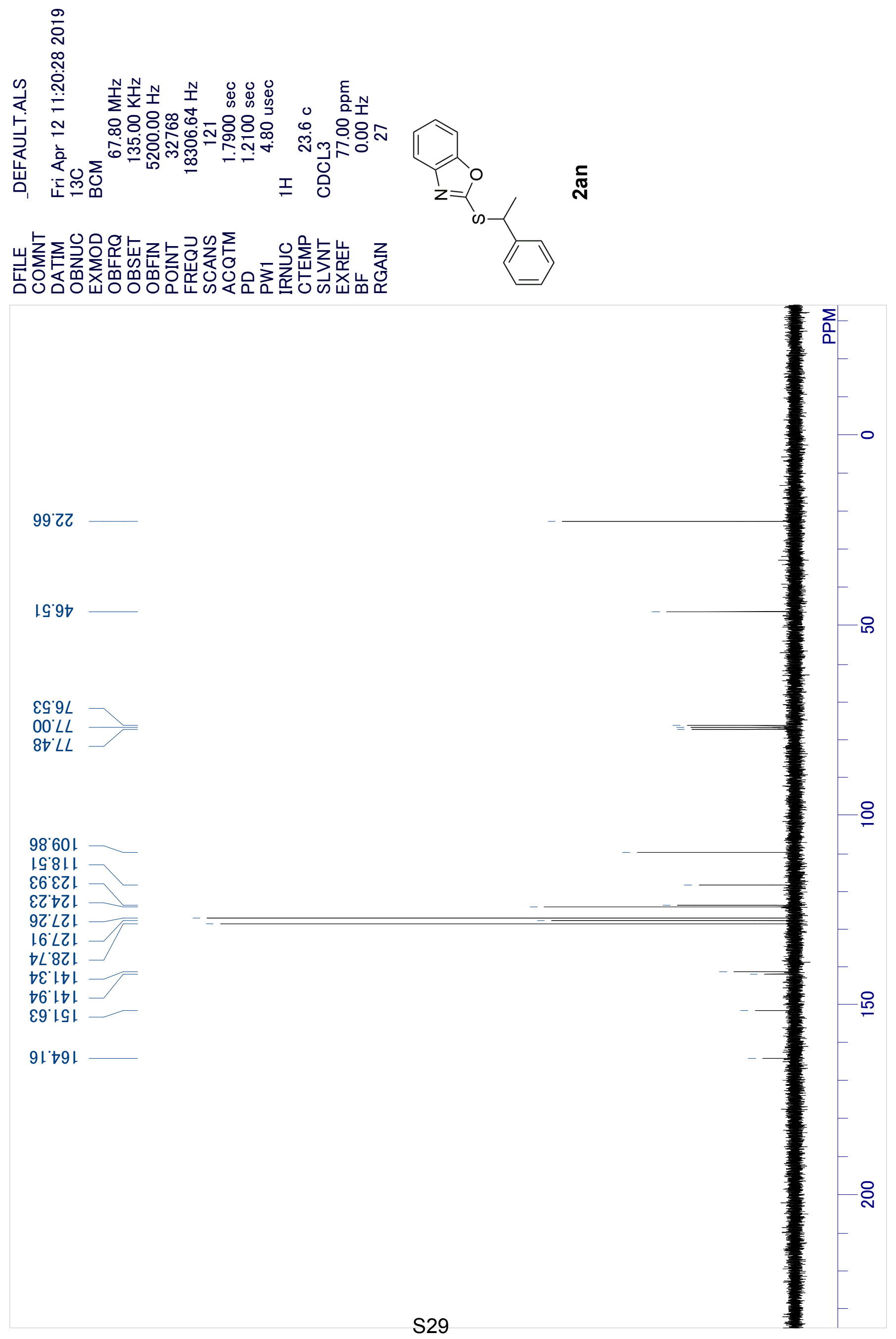




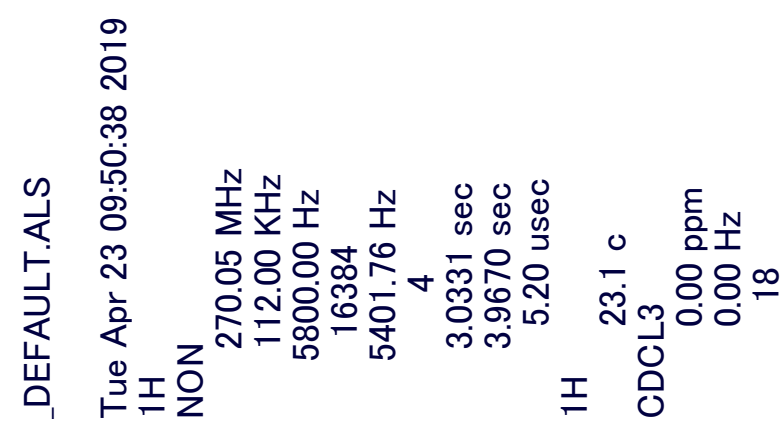

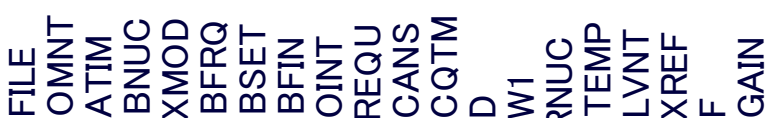

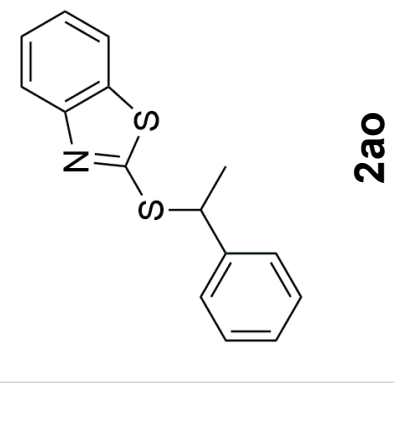

$00^{\circ} 0$

69

78 ।

98.1

ZL:

ㄴ.

$\angle L G$

619

$\varepsilon Z^{\prime} L$

$G Z^{\prime} L$

$9 Z^{\circ} L$

$8 Z^{\circ} L$

$I E^{*} L$

$\nabla \varepsilon^{\prime} L$

$9 \varepsilon^{\circ} L$

$8 \varepsilon^{\circ} L$

$I \nabla^{\circ} L$

$\nabla \nabla^{\circ} L$

9t'L

$6 \nabla^{\circ} L$

$\checkmark L L$

88 L

$26^{\circ} \mathrm{L}$ 


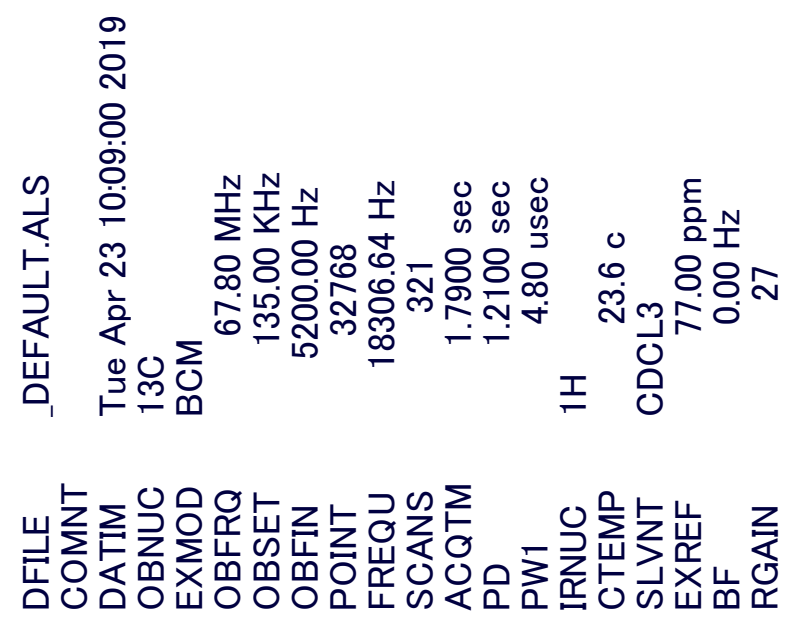

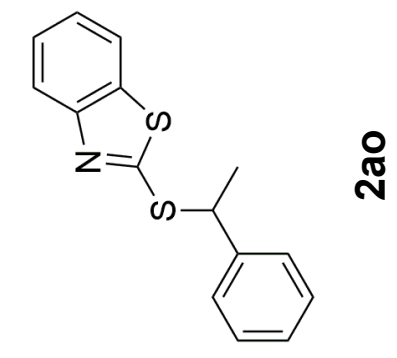

๑๐乙乙

$G G^{\circ} \angle t$

$\varepsilon G^{\circ} 9 L$

$00^{\circ} L L$

$87^{\circ} L L$

76.021

$0 L$ IZI

$0 \varepsilon ` \nabla Z 1$

86.9乙।

$6 \varepsilon^{\circ} \angle Z L$

9L'LZL

$\angle 9{ }^{\circ} 8 \mathrm{~L}$

08나

І乙๕૬।

\&8.99।

$\nabla Z \angle 6 L$

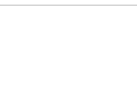

( 


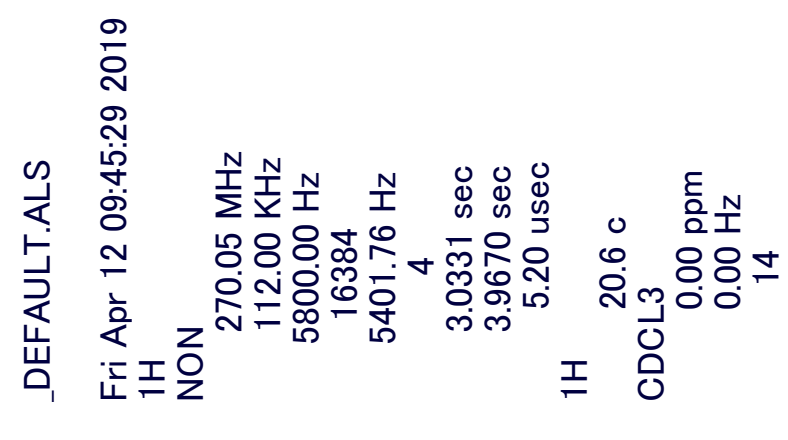

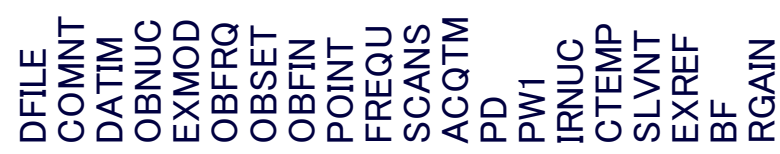
000

$99^{\circ}$

$69^{\prime \prime}$

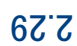

$0 \varepsilon^{\prime} 乙$

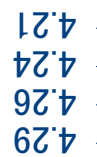

$10 \%$

$70^{\circ} \mathrm{L}$

$90^{\circ} \mathrm{L}$

$60^{\circ} \mathrm{L}$

$9 L^{\circ} \mathrm{L}$

$\angle H^{\circ} L$

$81 \cdot 1$

$8 \mathrm{~L}^{\circ} \mathrm{L}$

$6 \mathrm{~L} L$

$I Z L$

$I Z L$

ZZ'L

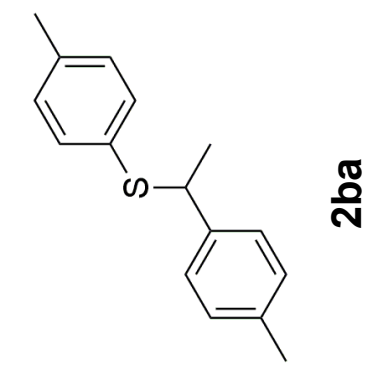

ㅇํำ

$\stackrel{q}{\varepsilon} t$ 


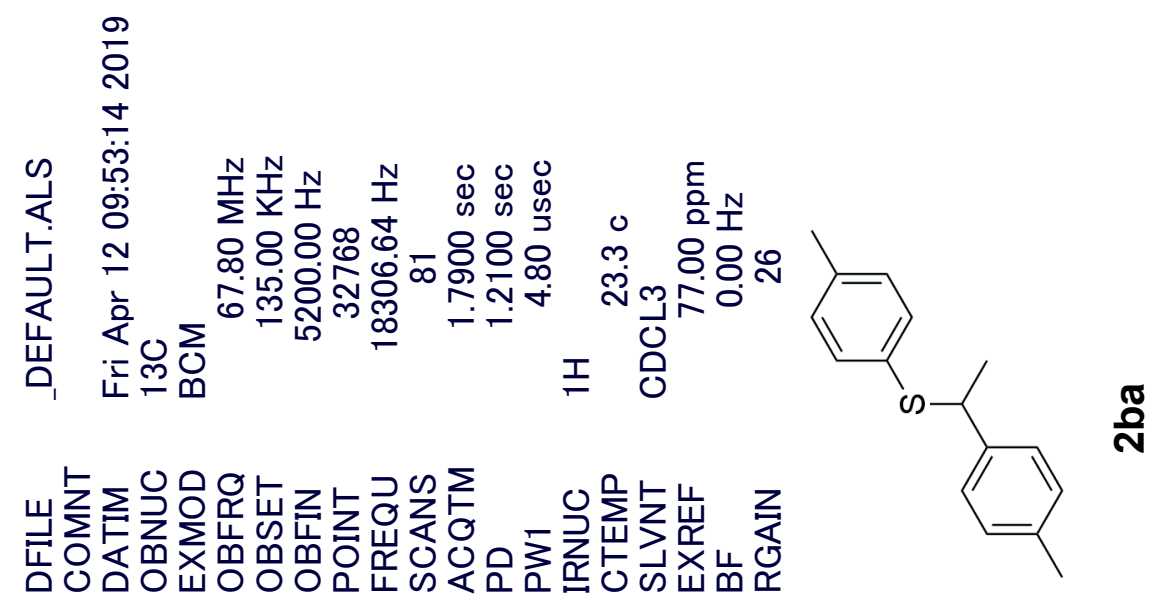

SOLI

$\angle 0^{\circ} \mathrm{LZ}$

$92 \mathrm{Z}$

$\angle 6^{\circ} \angle t$

ZG. $9 \mathrm{~L}$

00 LL

$\angle t L L$

II'LZI

00621

เナ6て

$\left|\mathcal{G}^{\circ}\right| \mathcal{E} \mid$

G6 Zह।

เ9. $9 \varepsilon \mid$

GI $L E$ L

9Z0t।

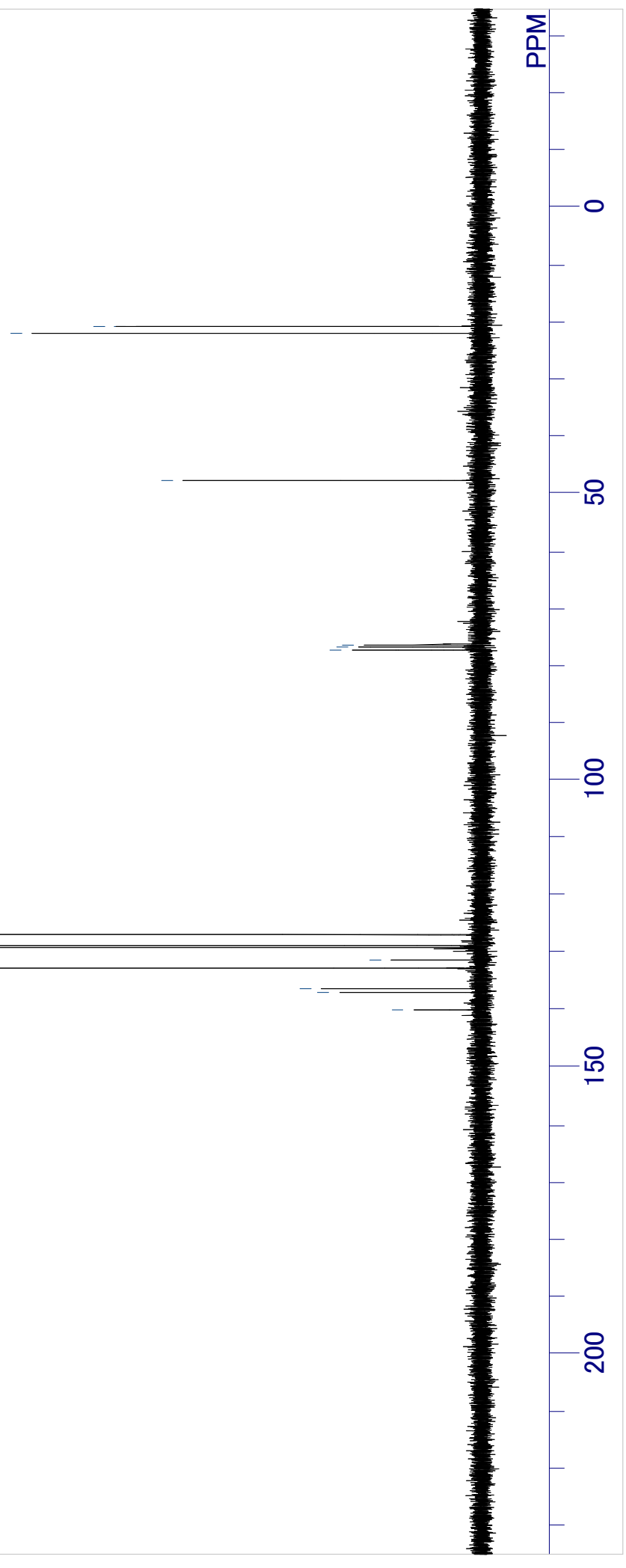




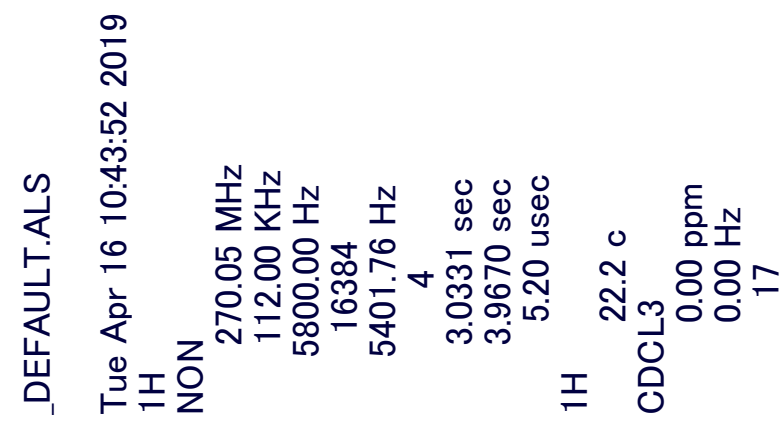

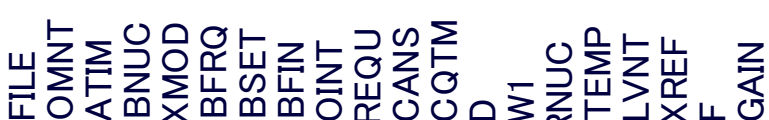

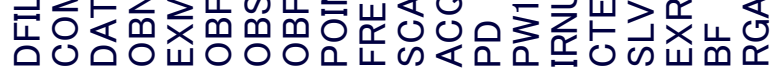

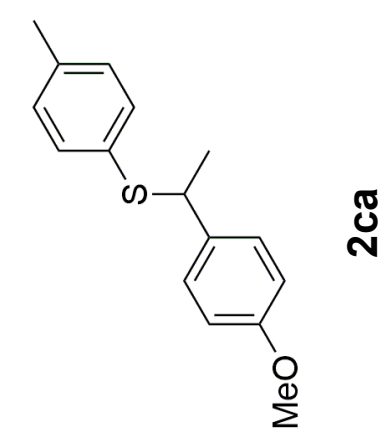

$00^{\circ} 0$

SG'।

99.

$69^{\circ}$ ।

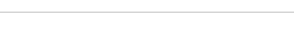

ป็

$0 \varepsilon^{\circ} 乙$

$8 L^{\circ} \varepsilon$

เで

七で

$9 Z^{\circ}$ t

$8 L^{\circ} 9$
$6 L^{\circ} 9$
$6 L^{\circ} 9$
$L 8 \cdot 9$
$Z 8^{\circ} 9$
$\varepsilon 8 \cdot 9$
$Z 0^{\circ} L$
$90^{\circ} L$
$8 L^{\circ} L$
$8 L^{\circ} \cdot$
$6 L^{\circ} L$
$L Z^{\circ} L$
$Z Z^{\circ} L$
$E Z^{\circ} L$
$G Z^{\circ} L$

टह

$\downarrow \bar{\varepsilon}$

(

$\sum_{0}-0$

乙'ह

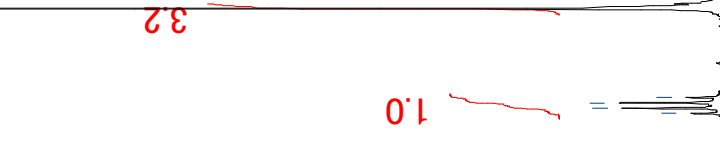



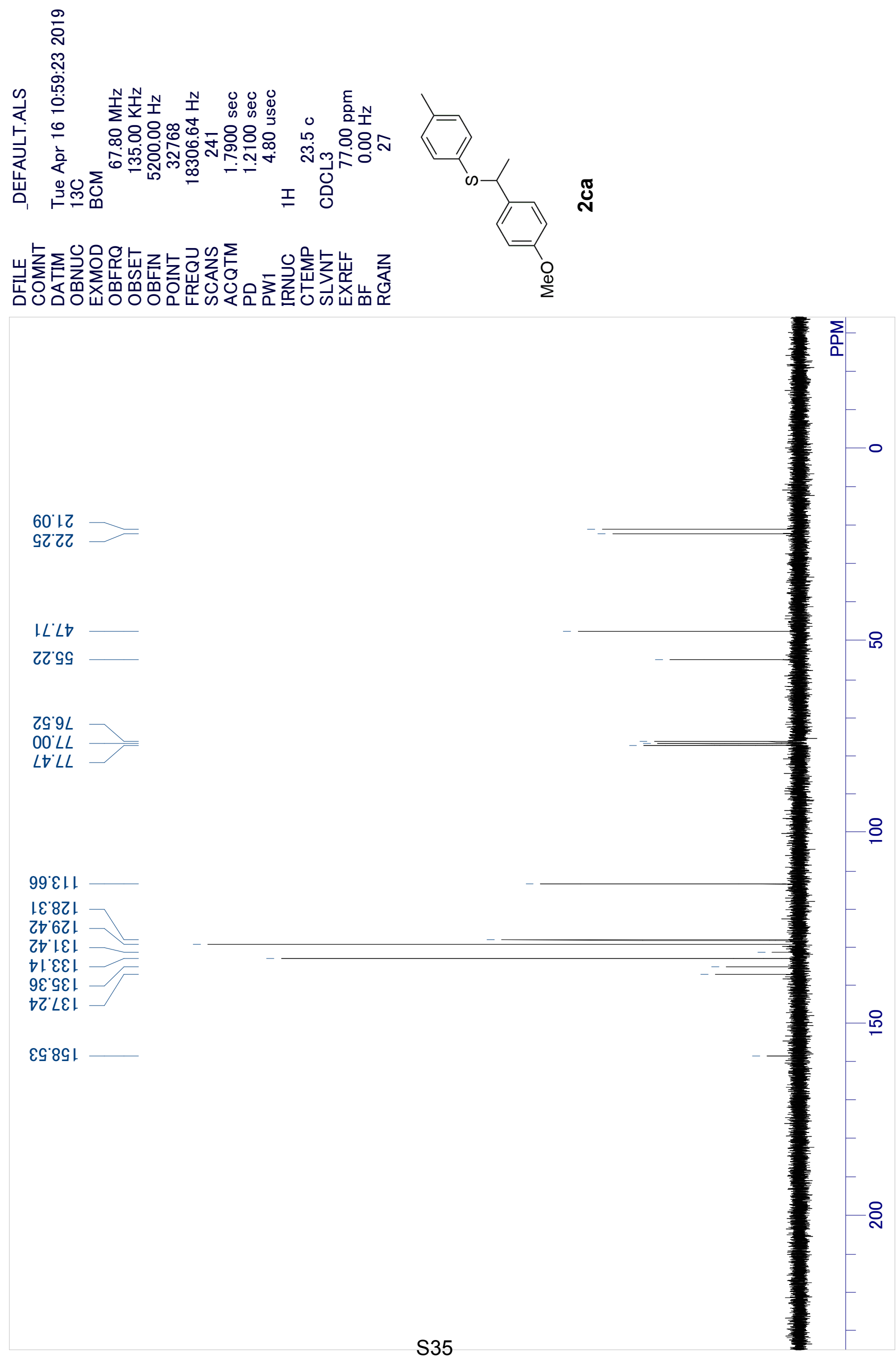


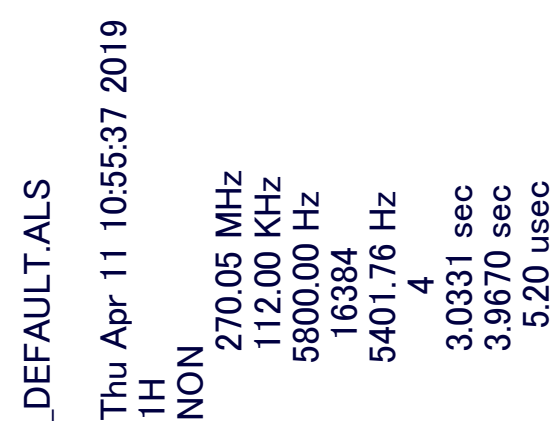

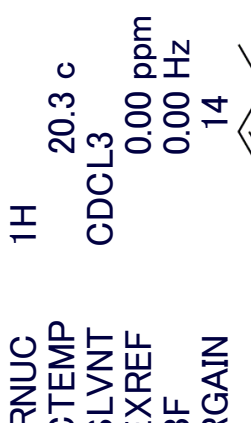

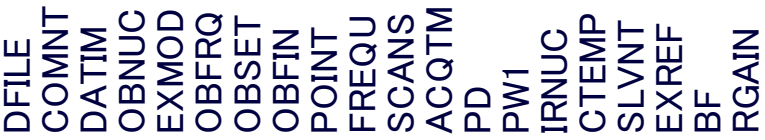

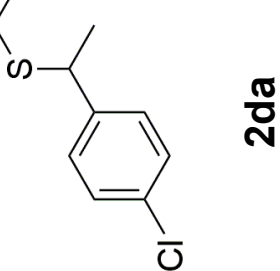

$00^{\circ} 0$

99.

$89^{\prime}$ เ

$6 乙 7$

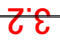

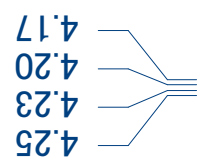

$0 \%$

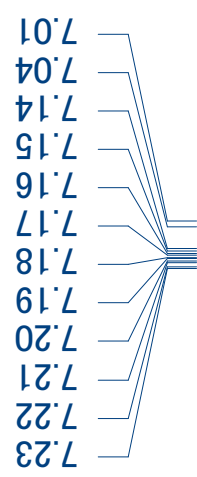

$9 \cdot 9$

$0 \%$ 

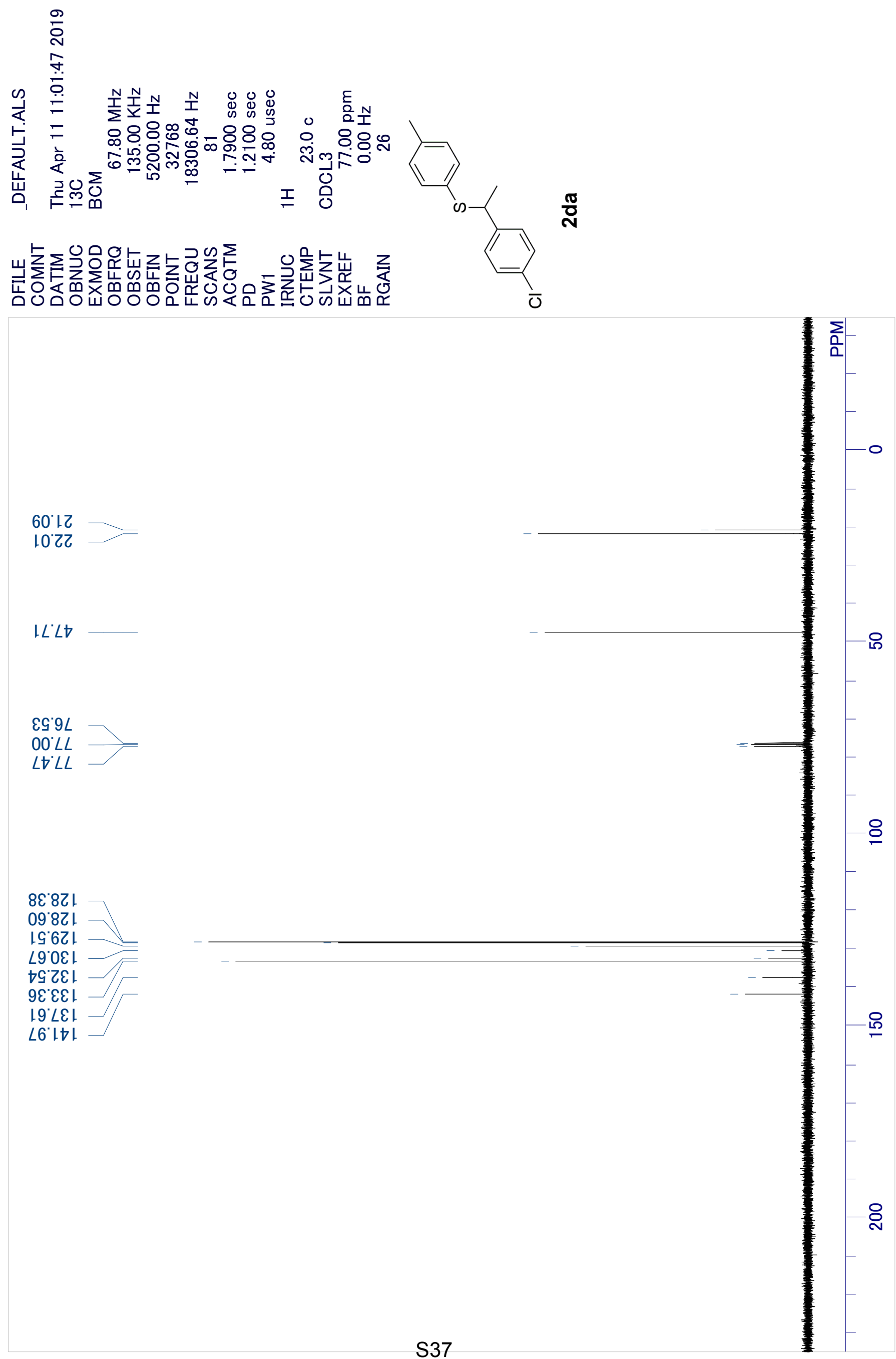


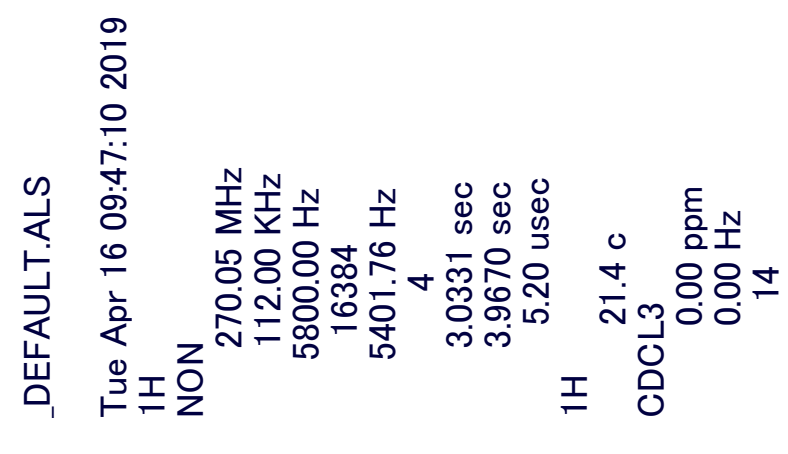

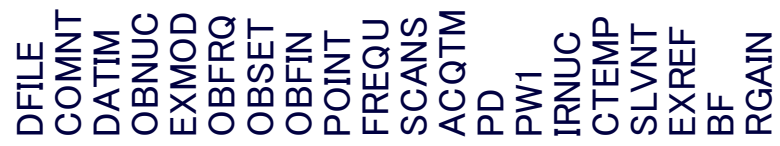

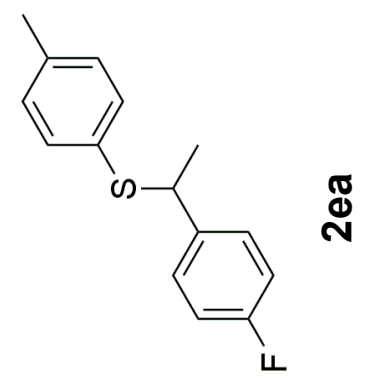

$00^{\circ} 0$

$99^{\circ}$ I

$69^{\circ} \mathrm{L}$

627

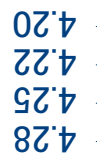

01

06.9

06.9

Z6.9

$\varepsilon 6.9$

†6.9

96.9

$96^{\prime \prime} 9$

$10 \%$

$70^{\circ} \mathrm{L}$

$\rightarrow L^{\circ} L$

GIL

GIL

LIL

$8 L^{\circ} L$

$81 \%$

$6 L^{\circ} L$

$0 Z$ 'L

IZ'L

ZZ L

$\varepsilon Z^{*} L$ 

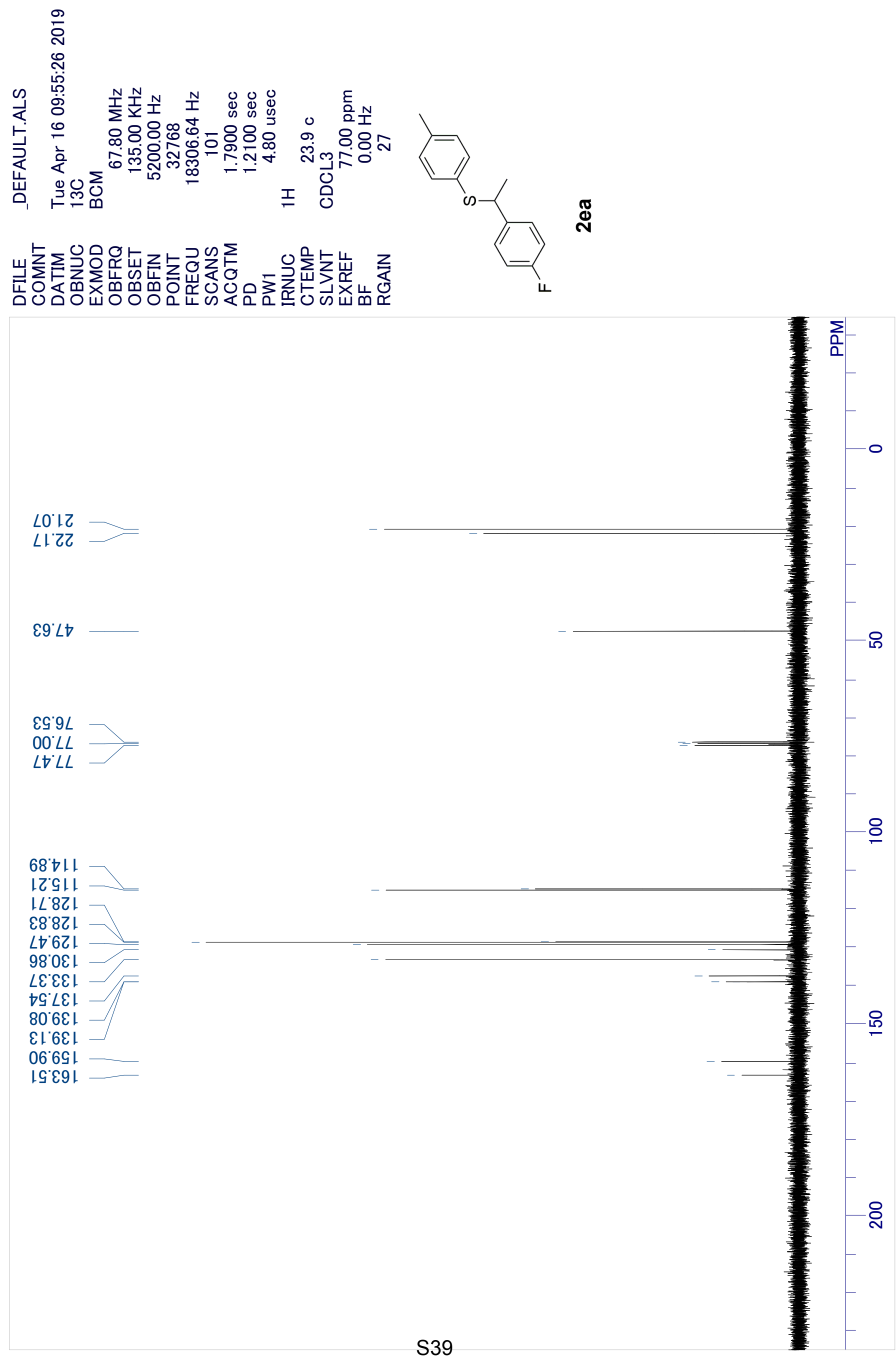


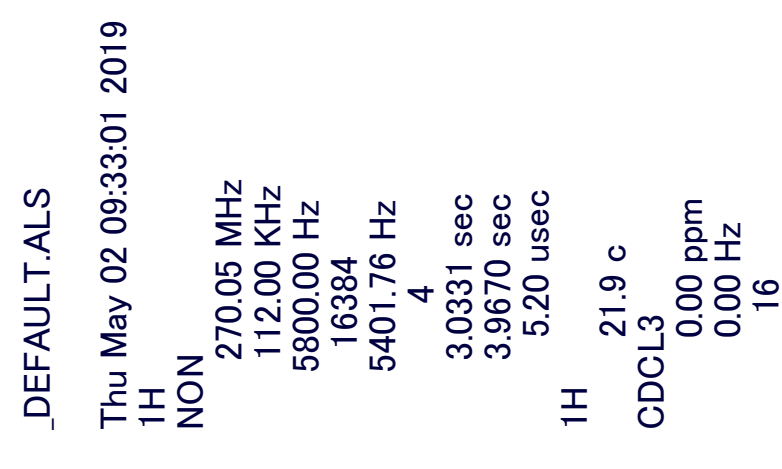

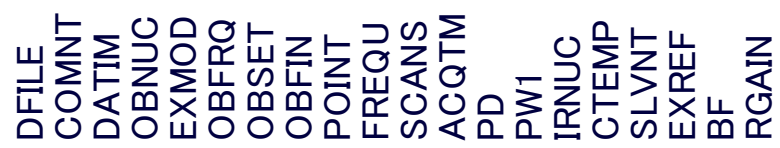

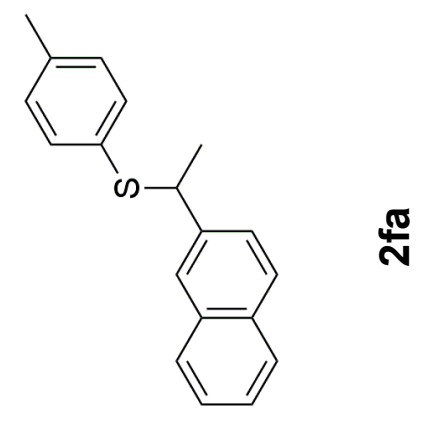

$00^{\circ} 0$

$\angle 9^{\circ} L$

$0 L^{\circ} L$

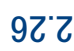

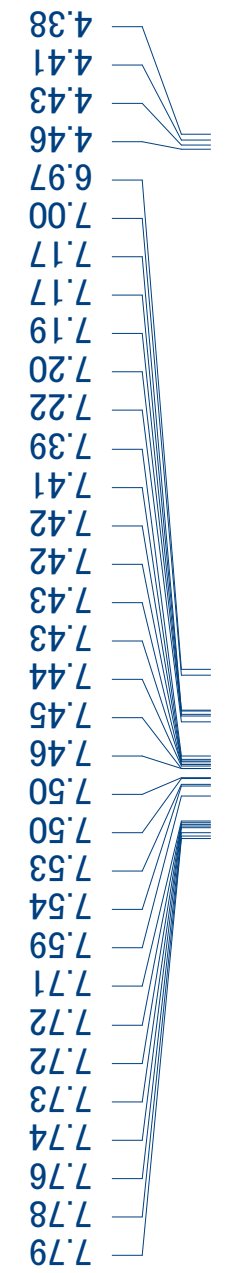

$\varepsilon \mathcal{E}$

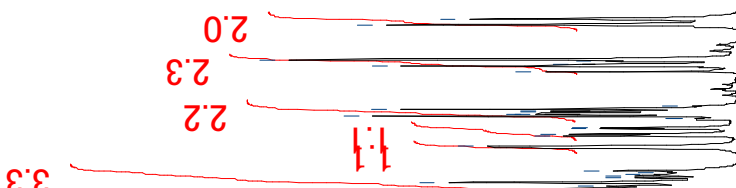




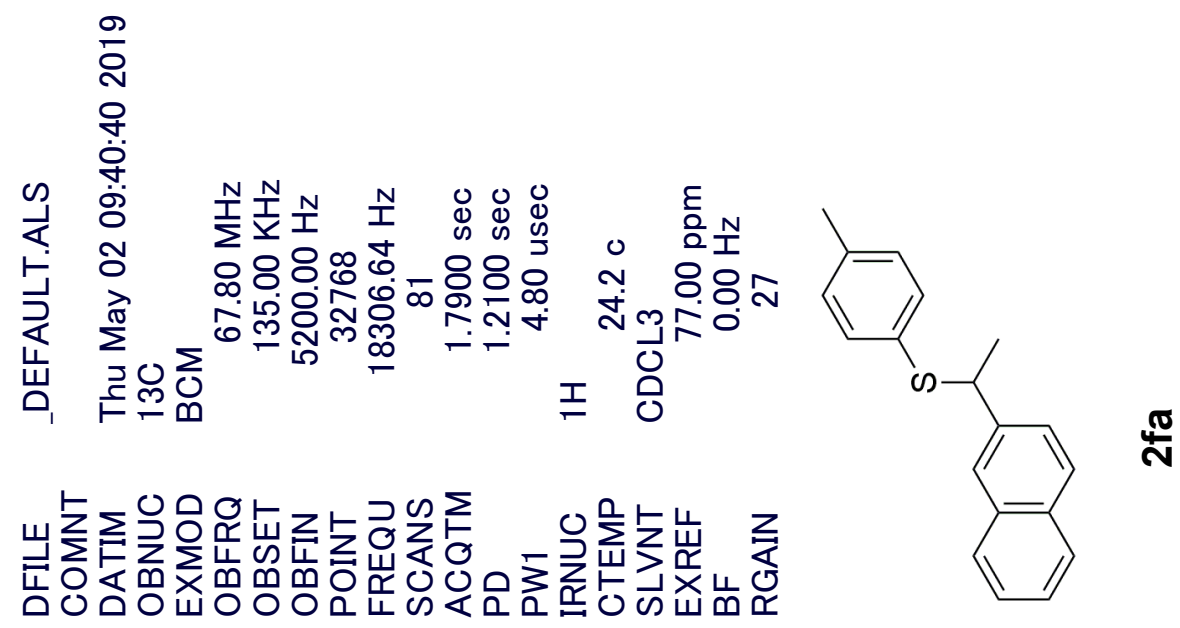

90.12
9.2Z

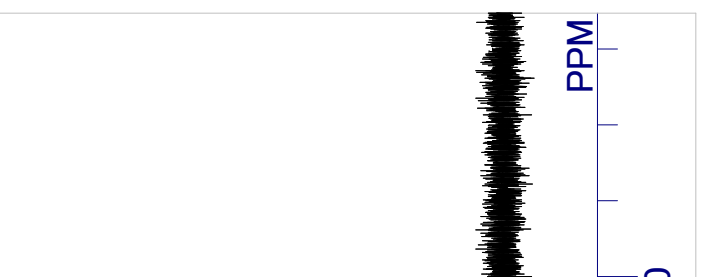

$69 \cdot 8 t$

$\varepsilon G^{\prime} 9 L$

$00^{\circ} L L$

$\angle \nabla^{\circ} L L$

$79^{\circ}$ Gद।
$89^{\circ}$ Gद।

L $L$ GL।

G6.9Z।

99ं $L Z$ L

LL'LZL

ㄴ.'8Z1

晌 62

EL'LL

乙9 ZE

9 เ $\varepsilon \mathcal{~}$

6เ $\mathcal{E}$ เ

$\angle \varepsilon^{\circ} \angle \mathcal{E} L$

$\varepsilon L^{\circ} 0 t \mid$ 


$$
\begin{aligned}
& \text { ลे }
\end{aligned}
$$

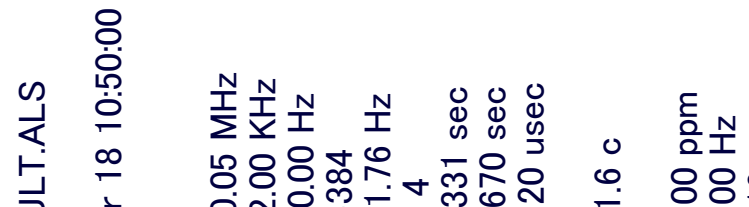

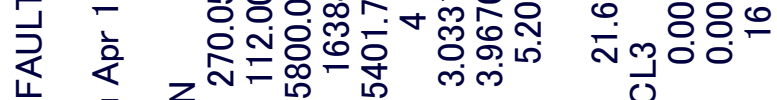

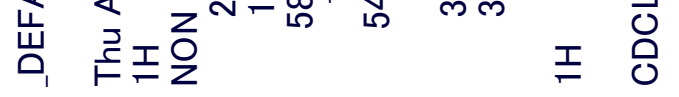

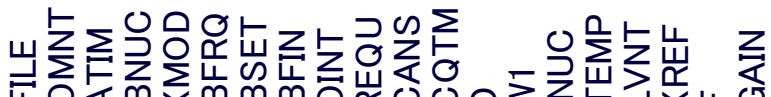

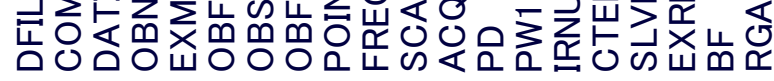

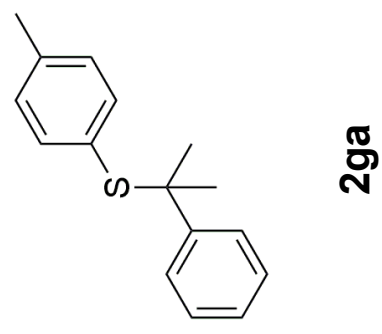
$00^{\circ} 0$

$\angle 9^{\circ}$ |

$6 乙 7$

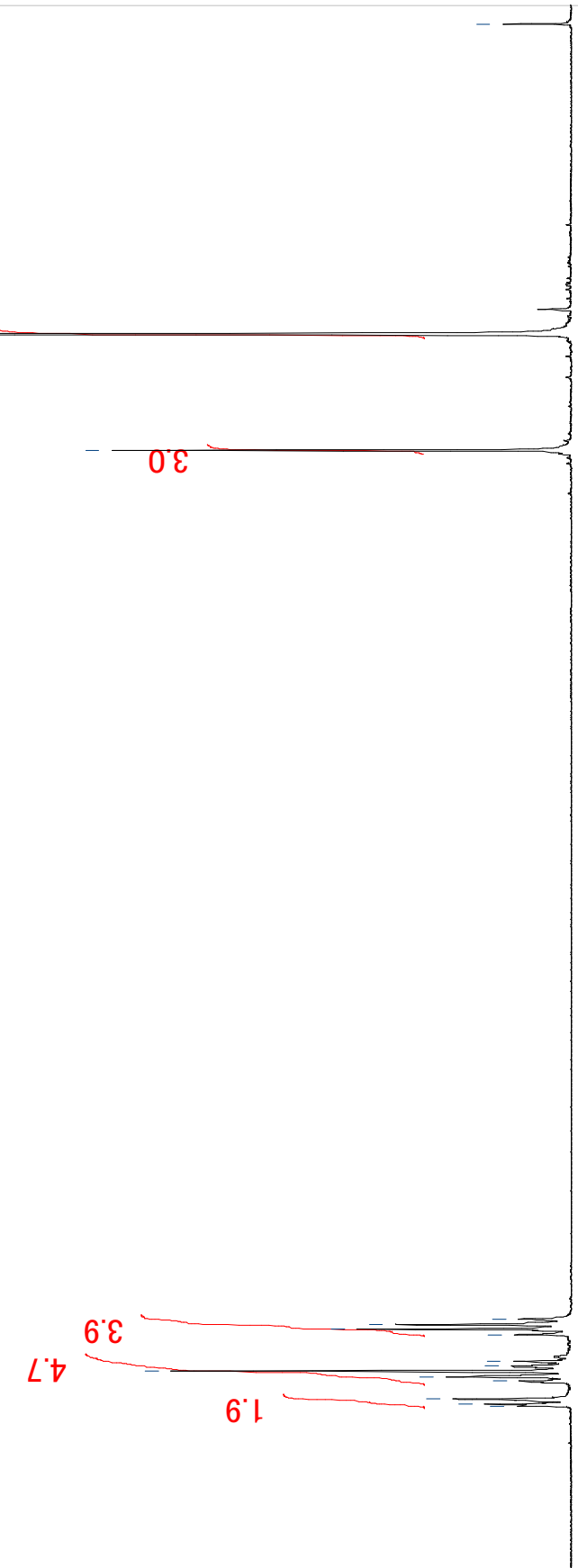

$0 \varepsilon^{\circ} L$

$6 \varepsilon^{\circ} L$

Z†'L

$\varepsilon \nabla L$ 


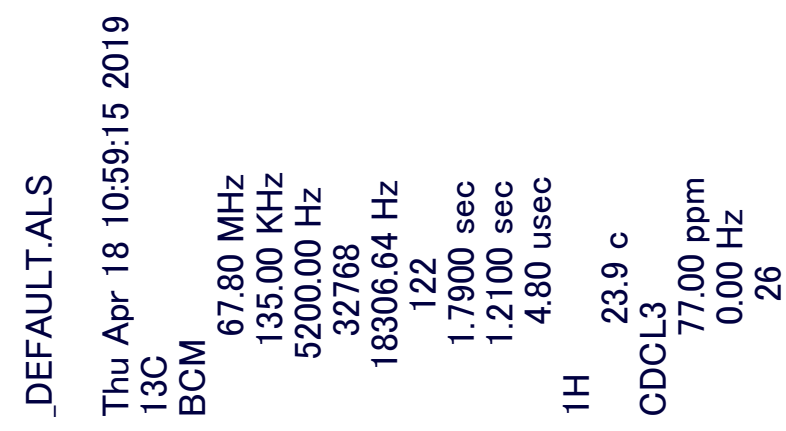

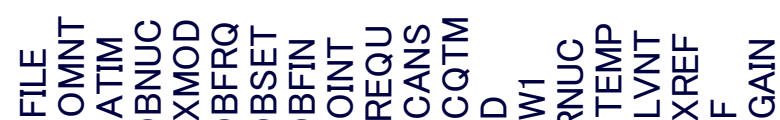

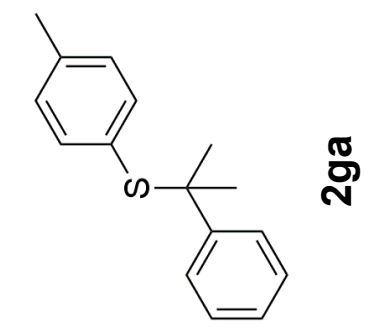

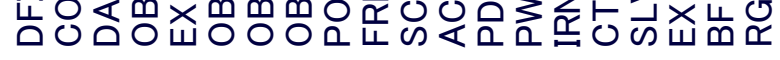

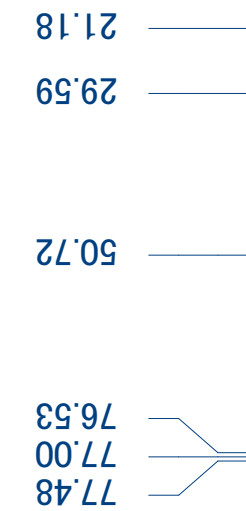

†७ 9 ᄂ

GG'9ZL

†8 LZL

70621

Gで6ZL

$69 \cdot 9 \varepsilon$ เ

†9.8 1

st9t 


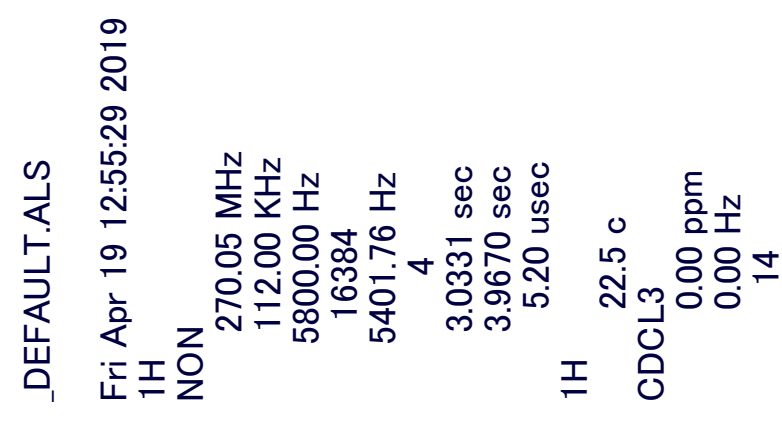

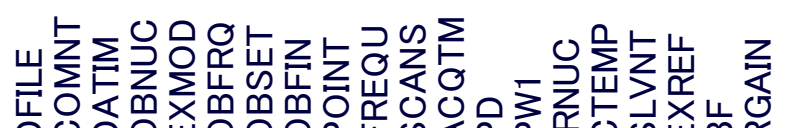
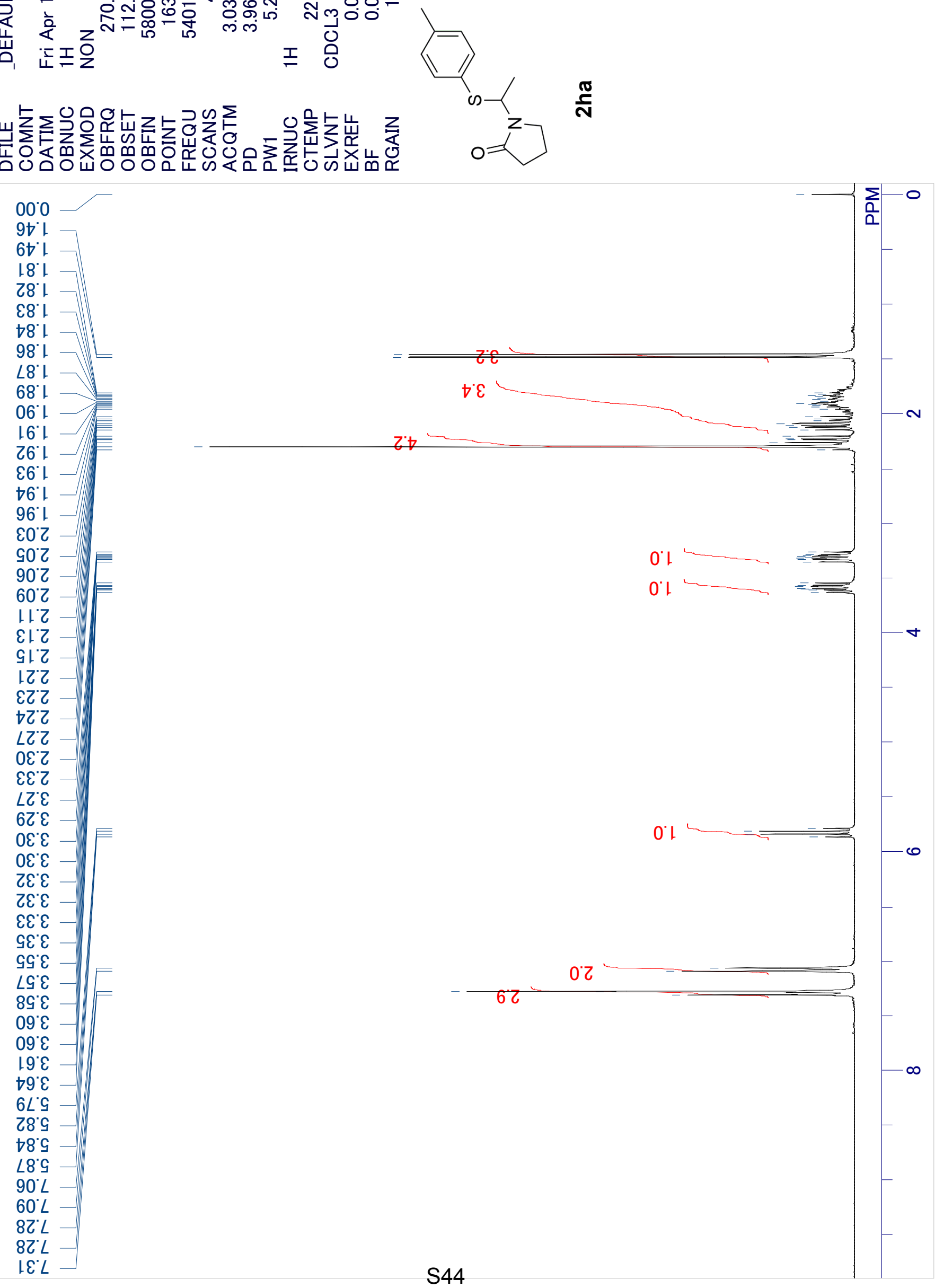


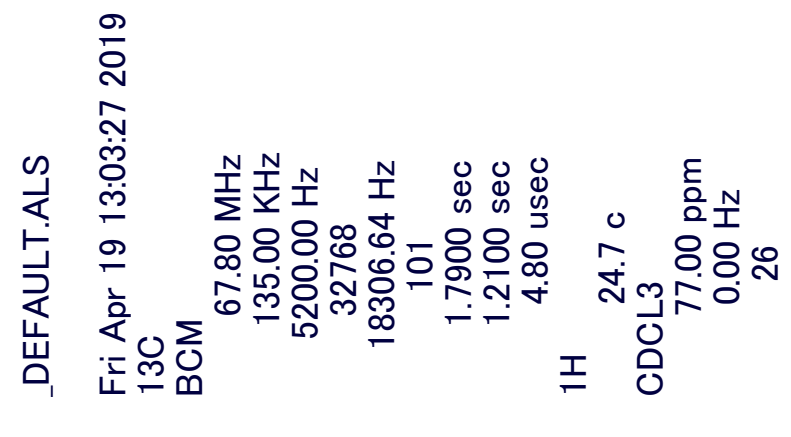

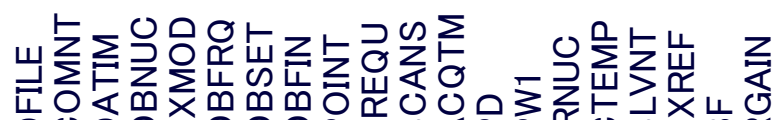

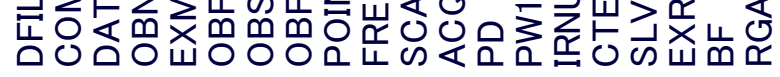
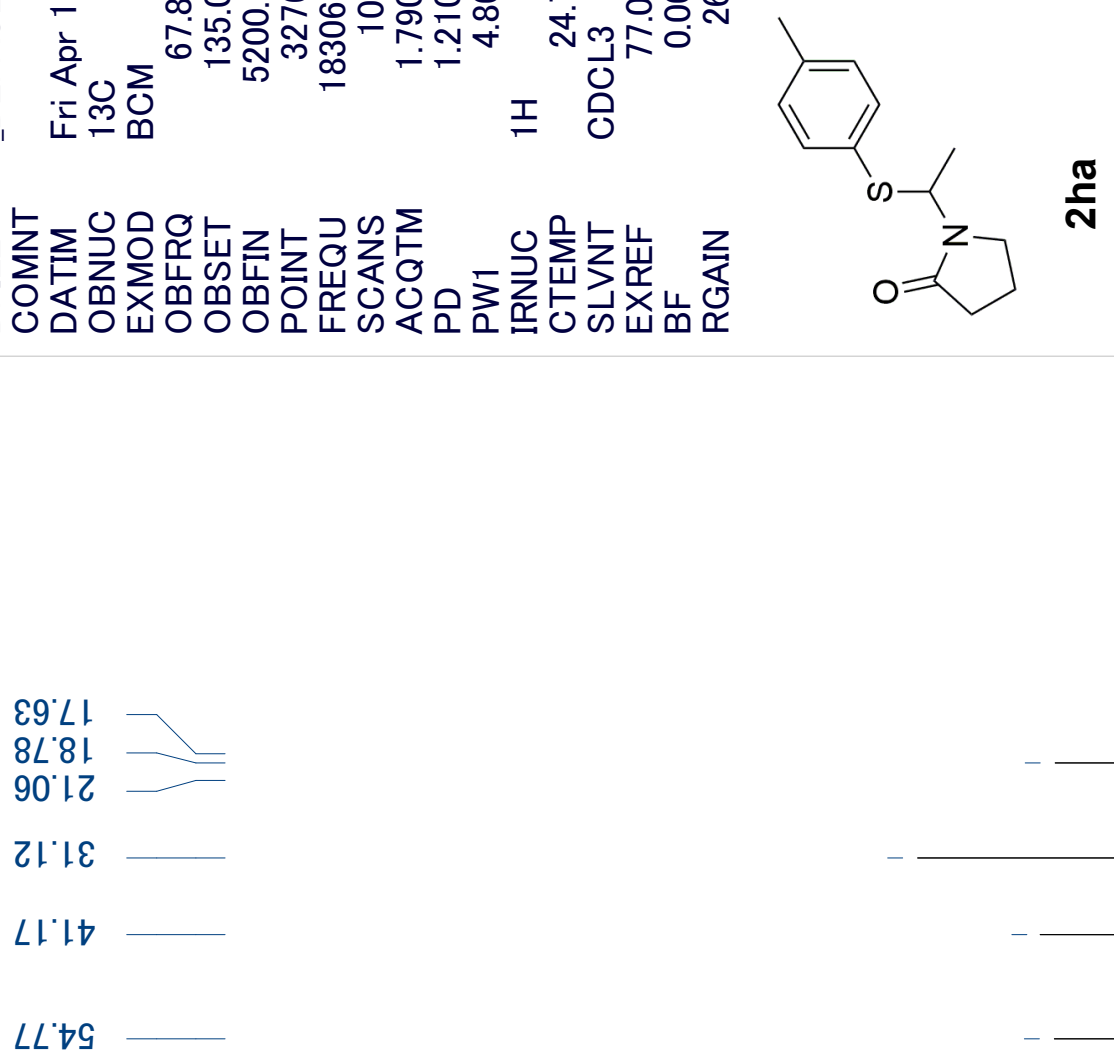

ZS. $9 \mathrm{~L}$

00 LL

$\angle t L L$

乙৷6て।

$\varepsilon 962$ ।

08 'Z ।

$\varepsilon L L^{\prime} L E L$

$\varsigma \varepsilon^{\circ} \succcurlyeq L L$ 


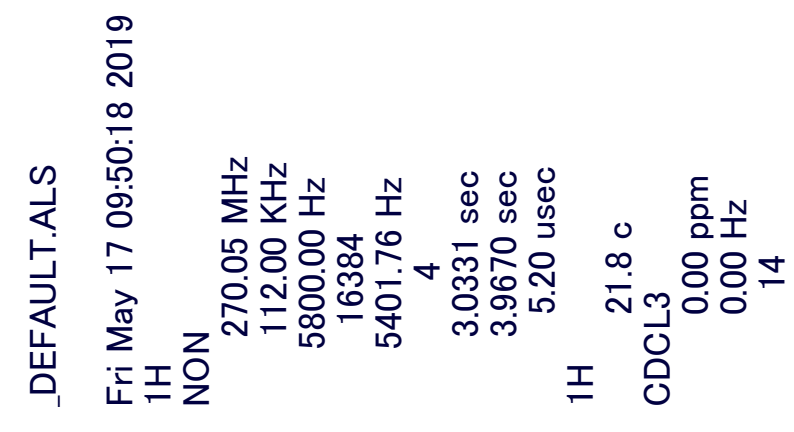

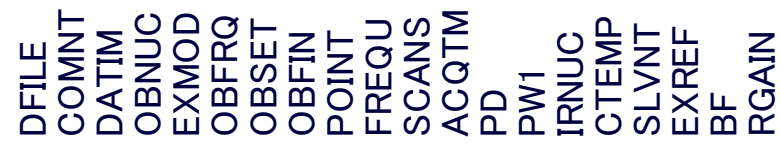
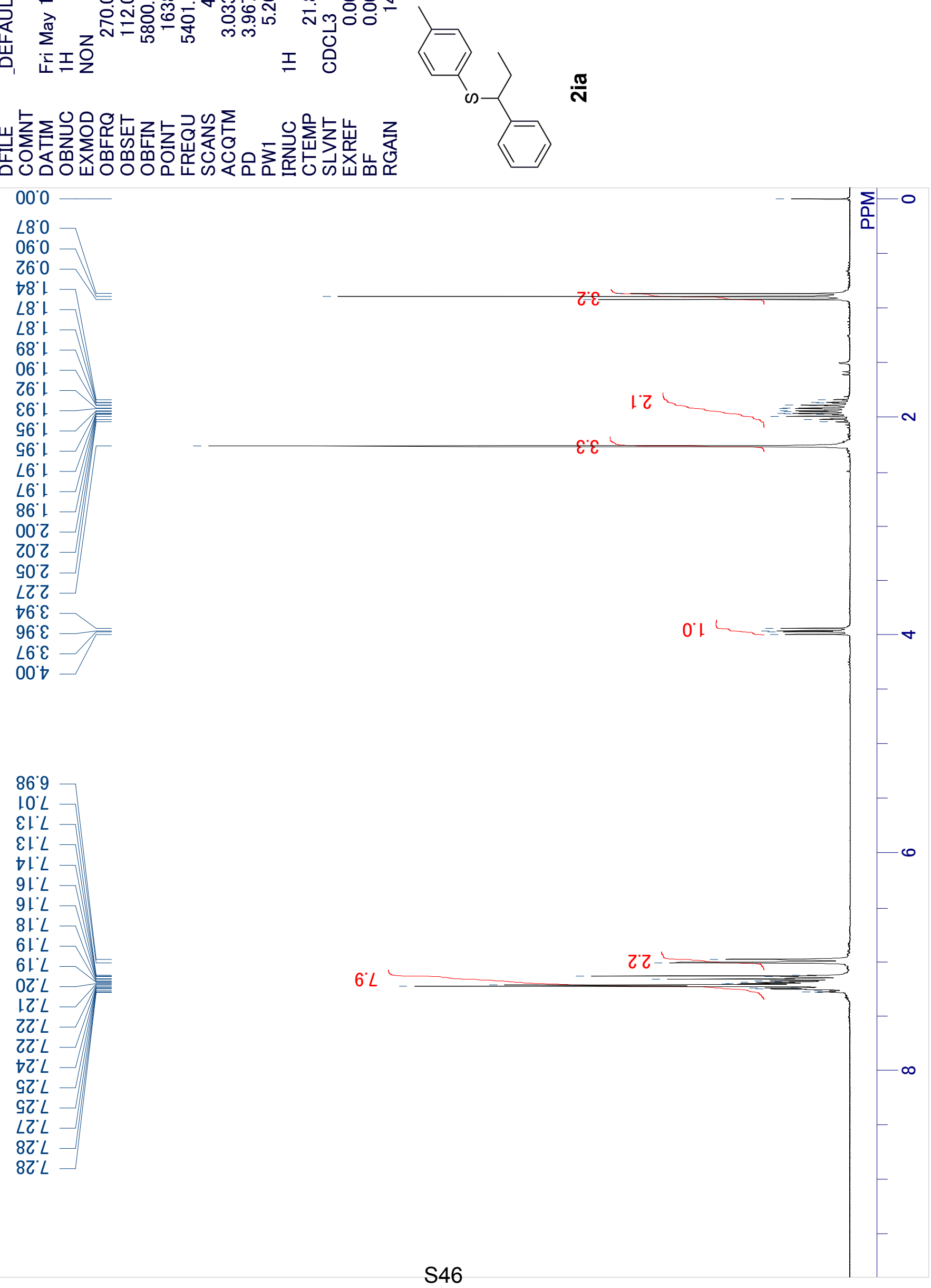

86.9

$10 \%$

$\varepsilon L^{\circ} L$

$\varepsilon I^{\circ} L$

$\rightarrow L L$

$9 L^{\circ} L$

$9 L^{\circ} L$

$81 \mathrm{~L}$

$6 \mathrm{I}^{\circ} \mathrm{L}$

6I'L

$0 Z L$

IZ'L

ZZ L

$Z Z L$

$\nabla Z ' L$

$G Z^{\circ} L$

$G Z L$

$L Z ' L$

$8 Z L$

$8 Z^{\circ} L$ 


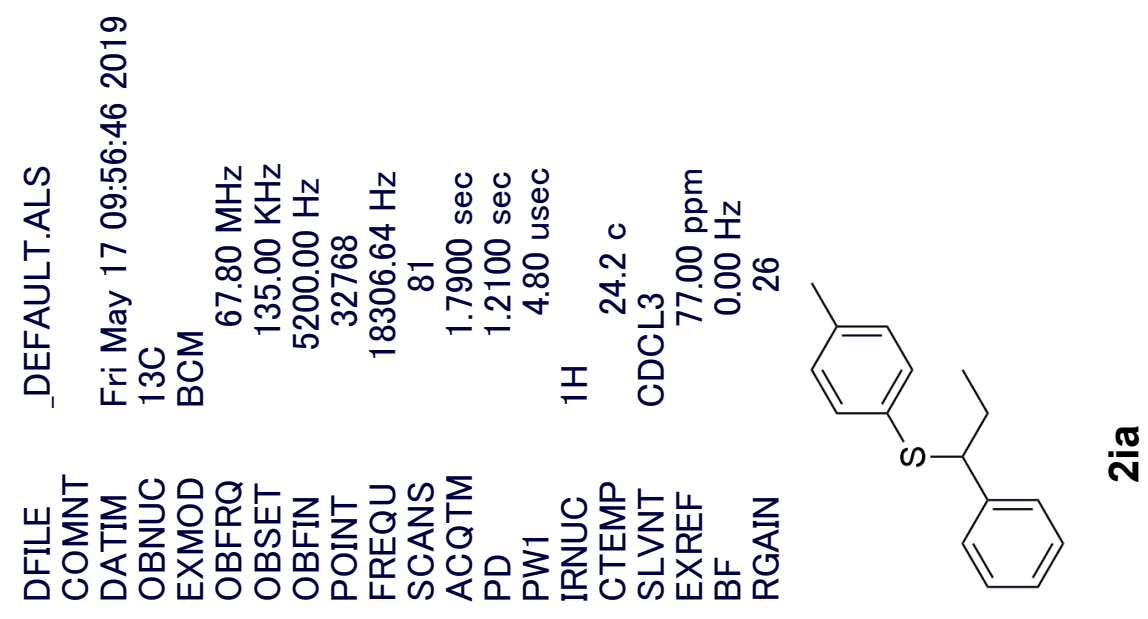

Gでレ

50.12

$\angle 1.62$

$\nabla L S G$

$\varepsilon G 9 L$

$00^{\circ} L L$

$8 t^{\circ} L L$

Z6.9Z1

$98^{\circ}$ LZI

IZ $8 Z$ ।

$\angle \varepsilon^{\circ} 6 Z$ L

$\varepsilon Z^{\prime}|\varepsilon|$

00 ¿E।

乙L'LE।

ยOてヤ।

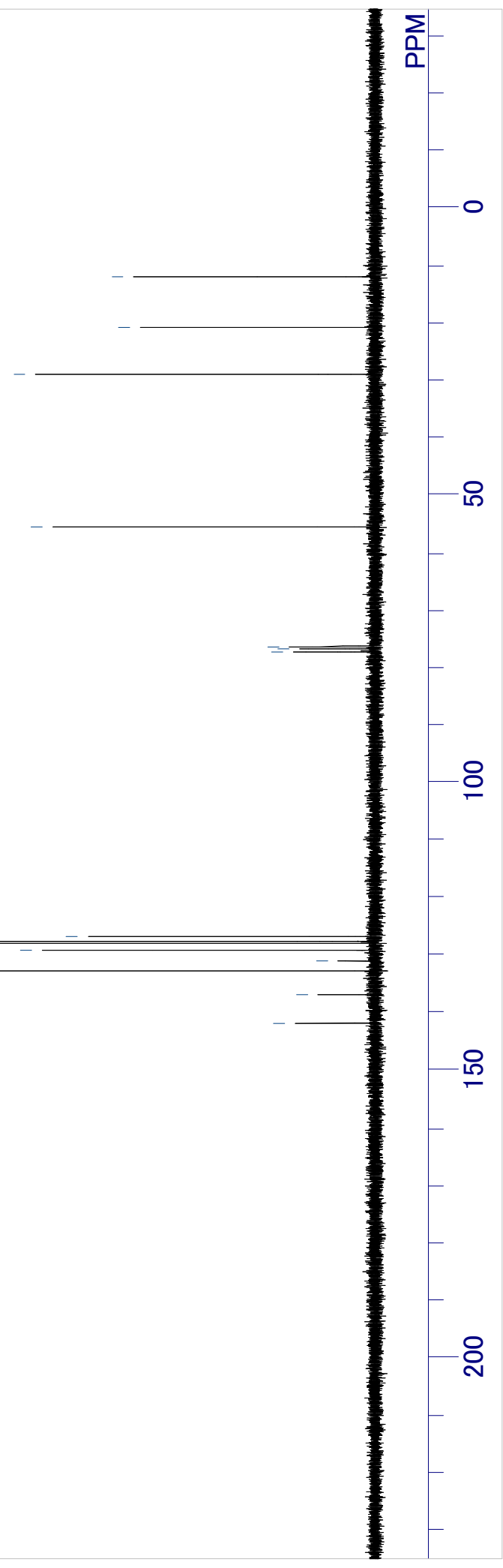




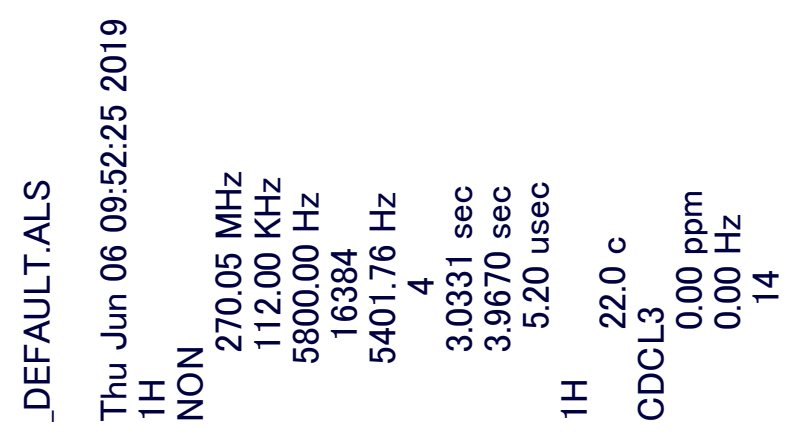

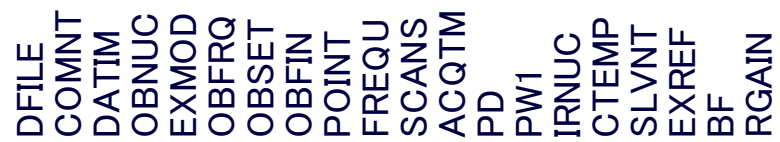

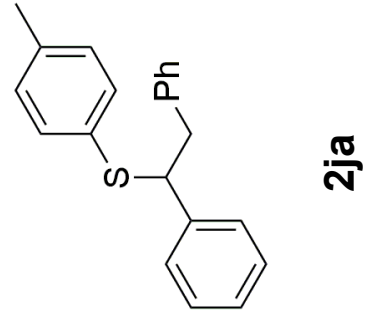

$00^{\circ} 0$

$L \nabla^{\prime}$

$\angle Z Z$

เ $\mathcal{L}$

†เ' $\mathcal{E}$

$9\llcorner\mathcal{L}$

$6 L^{\circ} \varepsilon$

I乙 $\varepsilon$

$\varepsilon \nearrow^{\prime} \varepsilon$

$9 \nearrow^{\prime} \varepsilon$

$8 \tau^{\prime} \varepsilon$

$8 Z^{\circ} t$

$0 \varepsilon \cdot \nabla$

$1 \varepsilon^{\circ} t$

$\varepsilon \varepsilon^{\circ} \dagger$

96.9

$\angle 6^{\circ} 9$

86.9

$66^{\circ} 9$

66.9

$00 \mathrm{~L}$

$10 \%$

$20 \%$

$80^{\circ} \mathrm{L}$

$60^{\circ} \mathrm{L}$

OL'L

ILL

ZIL

tI $L$

$G I L$

GI'L

9L'L

$\angle L L$

$8 L^{\circ} \mathrm{L}$

$6 \mathrm{I}^{\circ} \mathrm{L}$

$0 Z$ L

$0 Z$ L

IZL

ZZ'L

ZZ L 


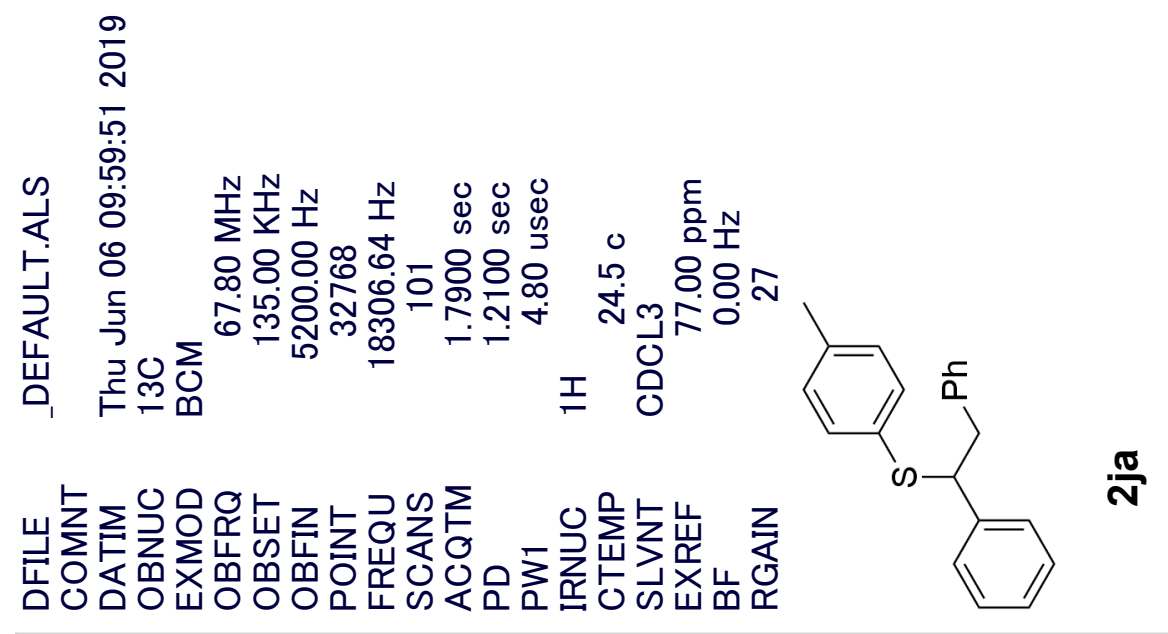

90.12

$29^{\circ} Z t$

0999

ZG. $9 \mathrm{~L}$

$00 \angle L$

$\angle t L L$

GZ9ZI

SO'LZI

20821

$608 \mathrm{ZL}$

9 |'8Z।

เ। 62 L

$\angle \forall 6 Z$ I

$9|\cdot| \varepsilon \mid$

เ6 ZE।

$9 Z^{\circ} \angle E L$

$\succ 88 \varepsilon$

$\varepsilon \varepsilon^{\prime}|t|$

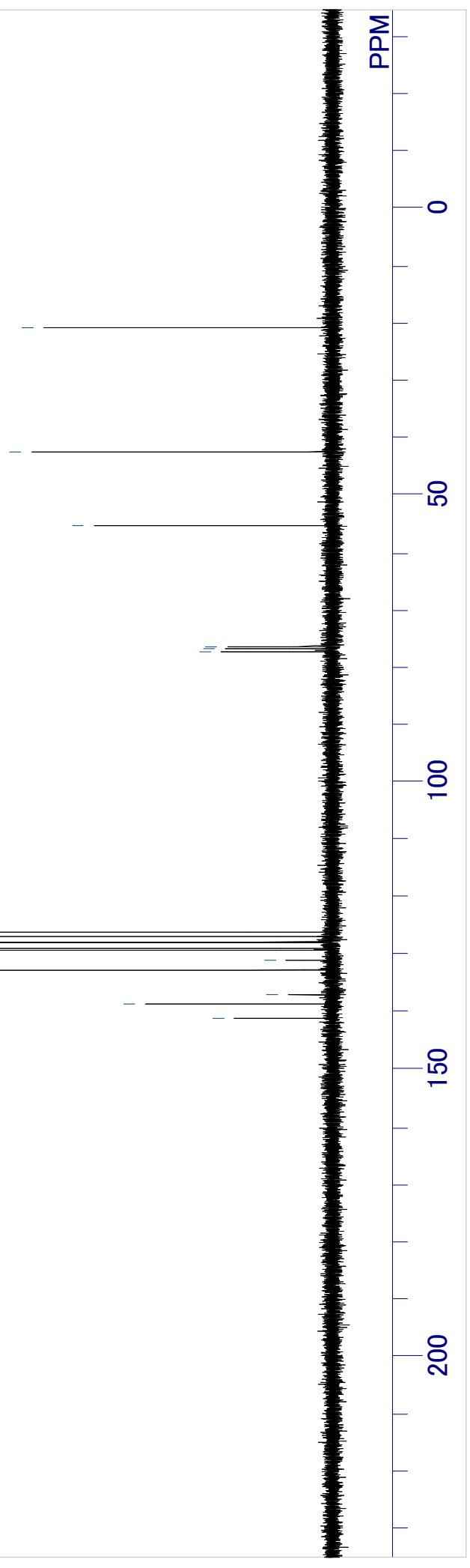




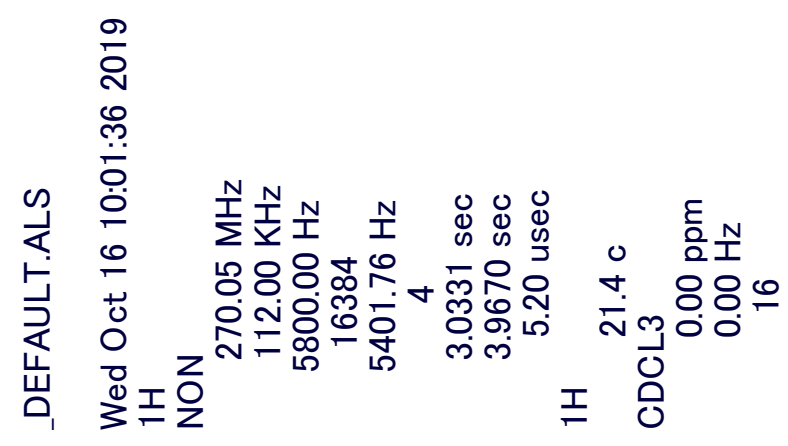

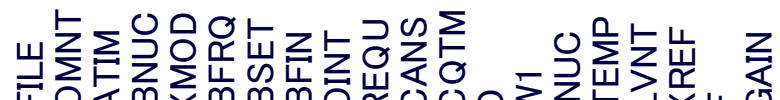

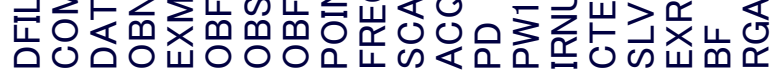
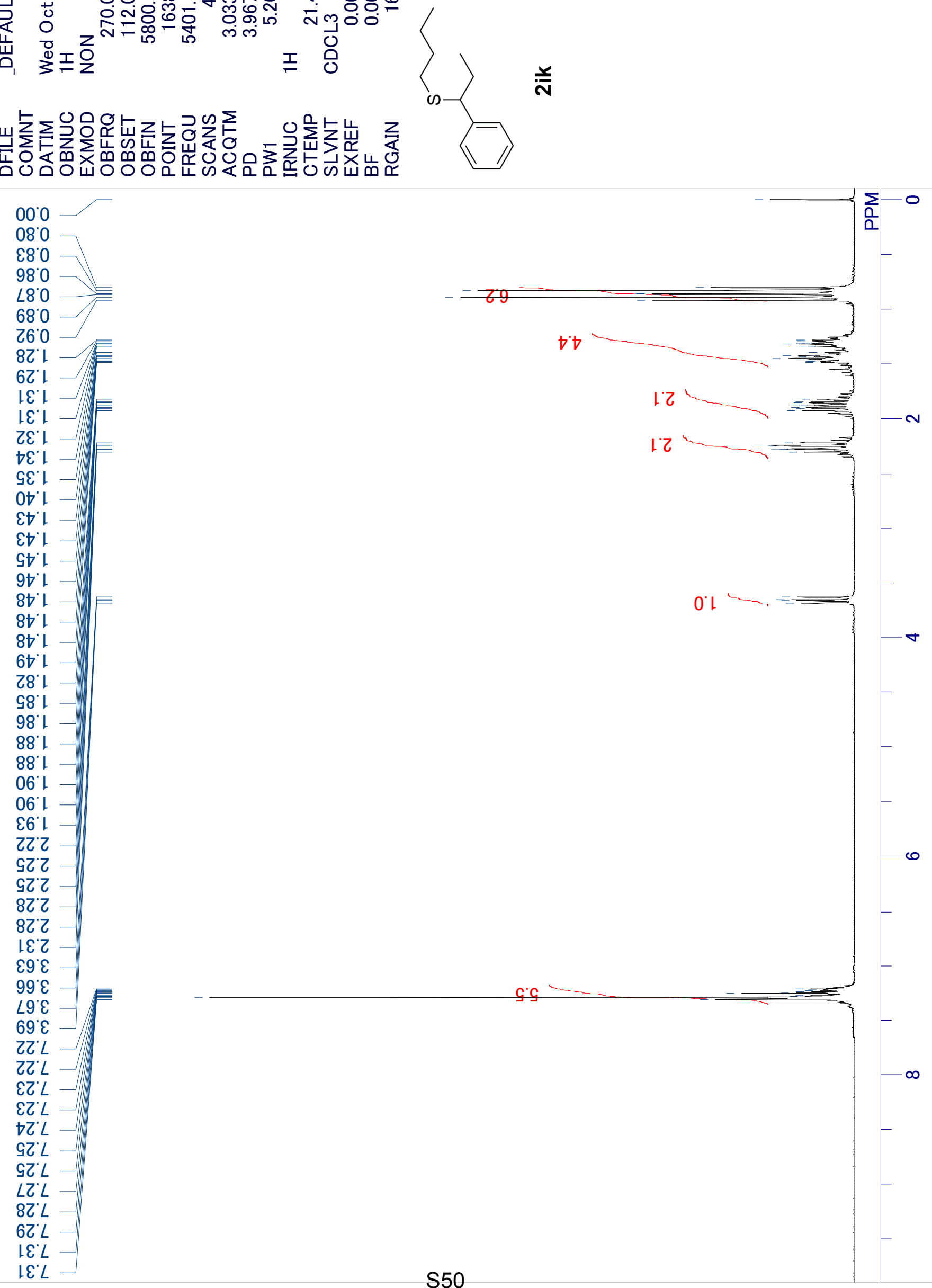


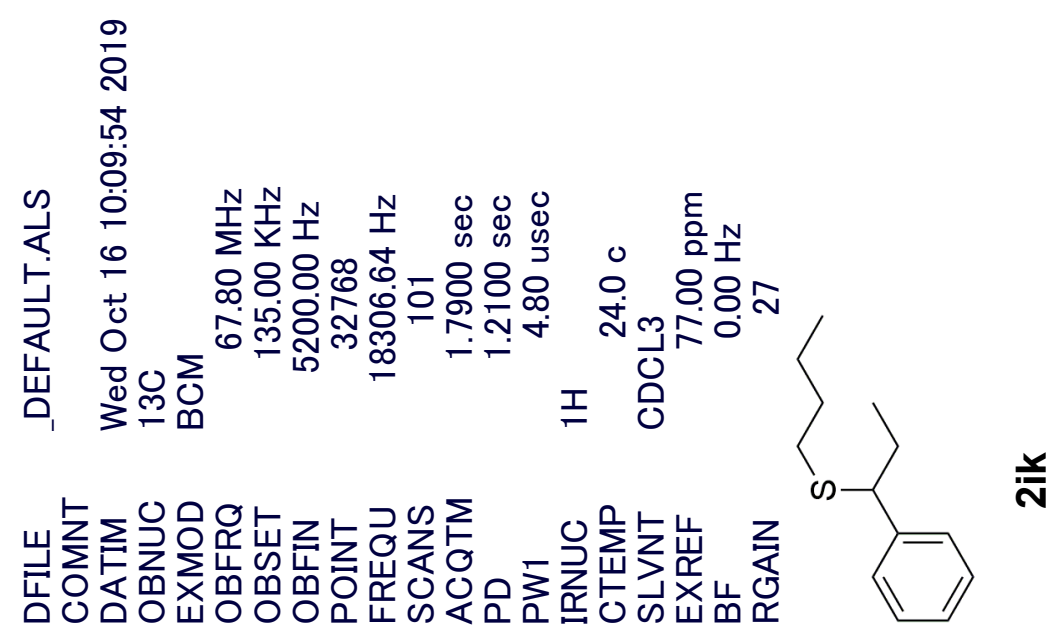

$\angle \varepsilon^{\circ} 乙 L$

乙9.

86.1乙

69.62

$99^{\circ} 0 \varepsilon$

$9 \nabla^{\circ} \mid \mathcal{E}$

DG'L

EG.9L

$00 \angle L$

$\angle \nabla^{\circ} L L$

†8 9 ᄂ

98 L乙L

6乙.8乙।

L8てヤ

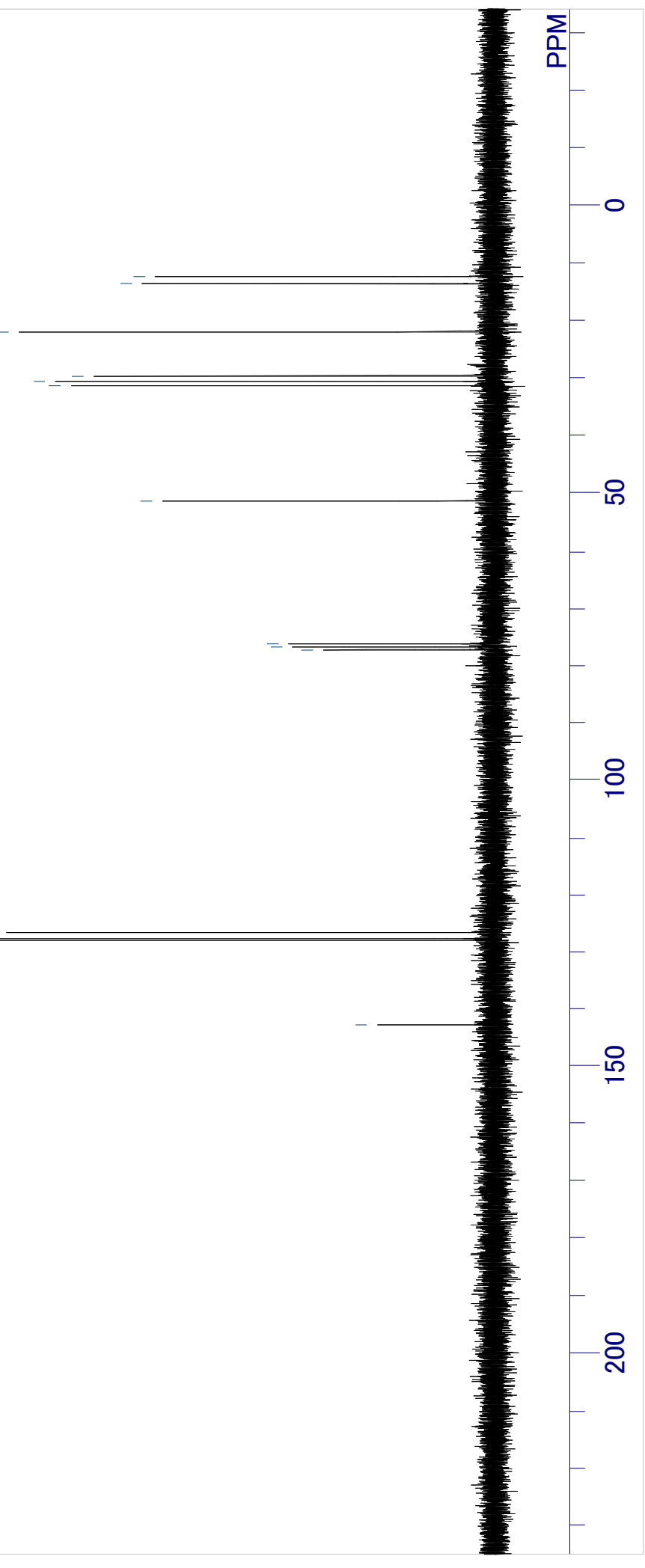




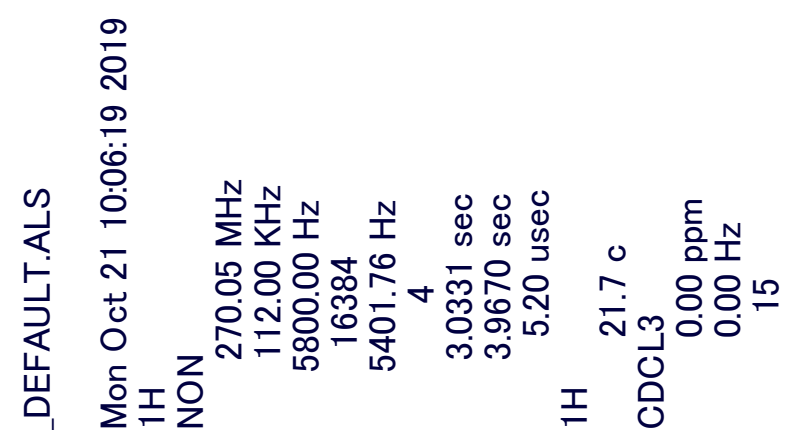

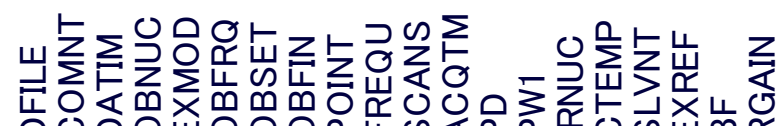
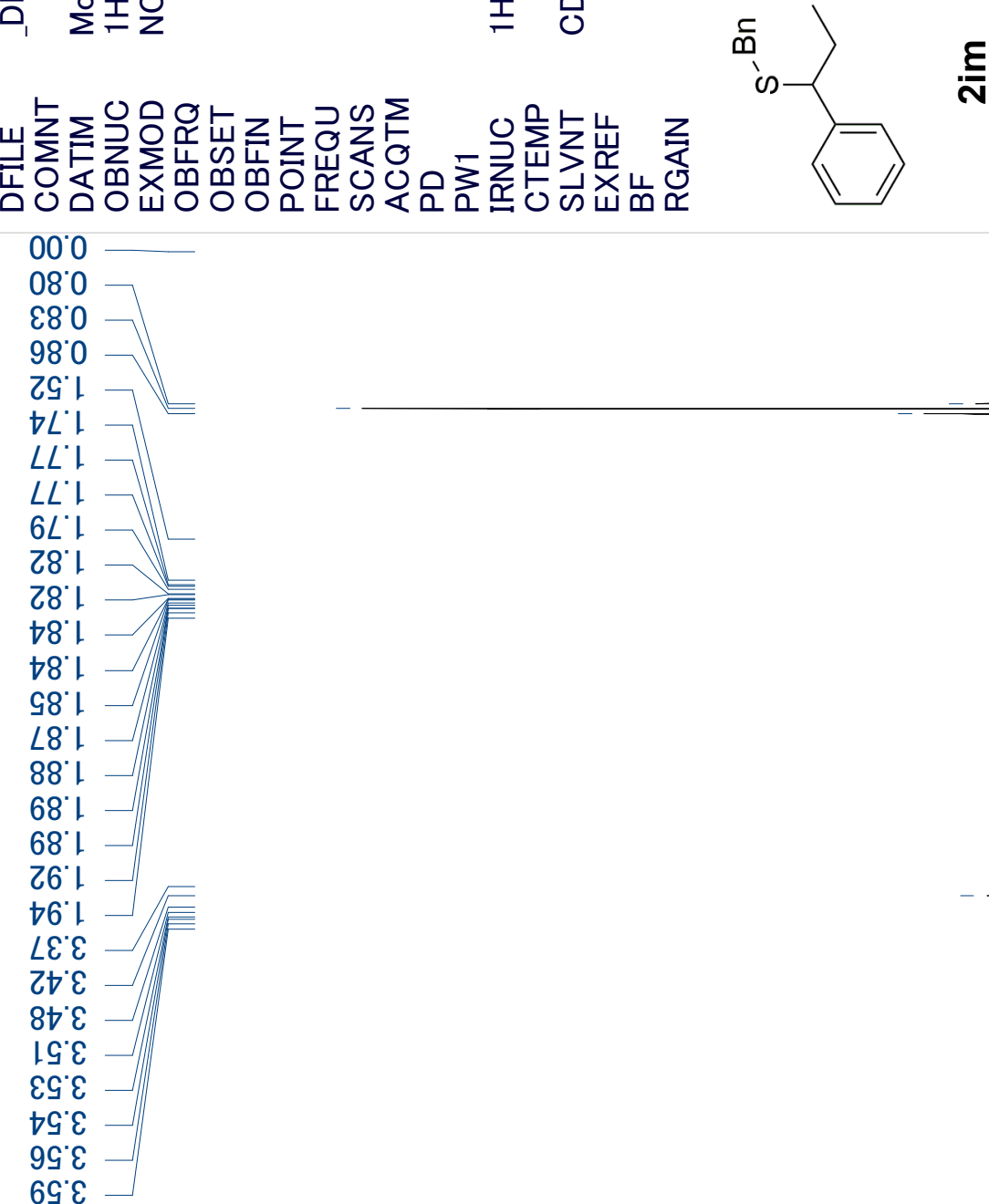

$6 I^{\circ} L$

IZ'L

$\varepsilon Z^{*} L$

$\nabla Z L$

$\nabla Z ' L$

G $L$

$L Z ' L$

$L Z \cdot L$

$8 Z^{\circ} L$

$6 Z^{\circ} L$

IE'L

乙E'L

$\varepsilon \varepsilon^{\circ} L$

$\varepsilon \varepsilon^{\circ} L$

$G \mathcal{E}^{\circ} L$

$9 \varepsilon^{\circ} L$

$9 \varepsilon^{\prime} L$ 


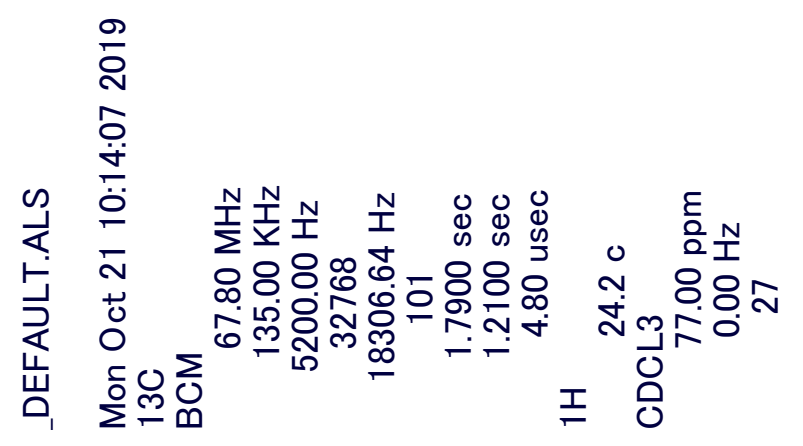

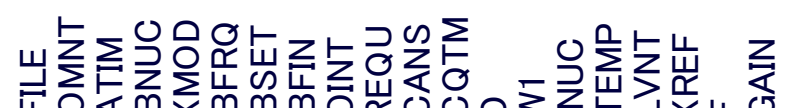

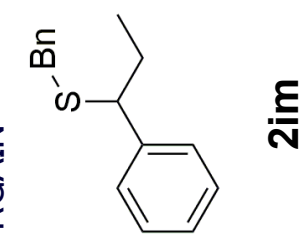

そでレ

$\varepsilon \mathcal{C}^{\prime} 62$

$\varsigma \mathcal{G} \subseteq \mathcal{E}$

2609

ZS. $9 \mathrm{~L}$

00 LL

$\angle t L L$

GL'9ZI

86.9Z1

II.8Z।

乙๕ $8 Z 1$

$9 \varepsilon 8 Z$ ।

$88^{\circ} 8 \mathrm{~L}$

$\angle \nabla^{\circ} 8 \mathcal{E}$ ।

8๕こてト

$\stackrel{\Sigma}{\text { N }}$ 


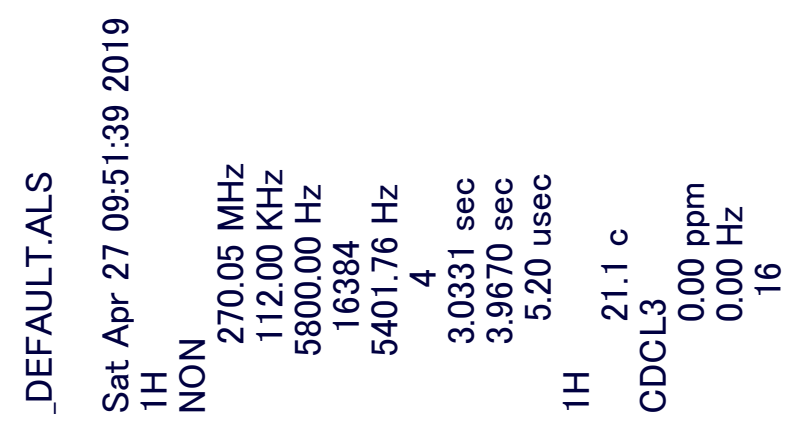

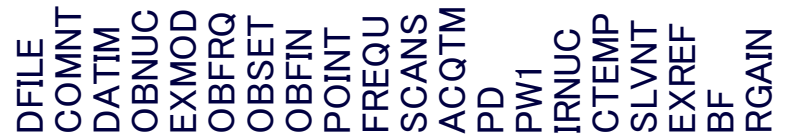
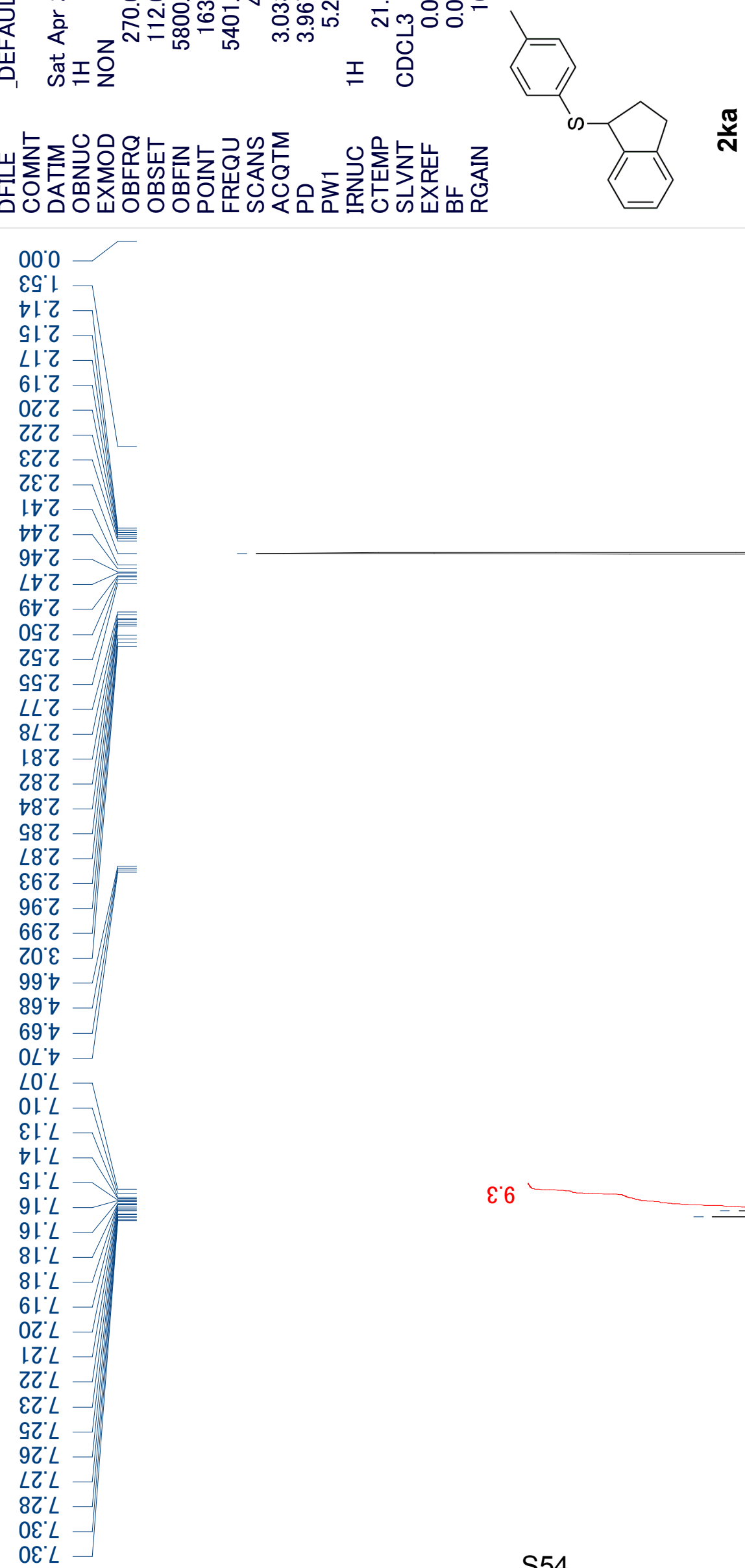

$\varepsilon 6$ 


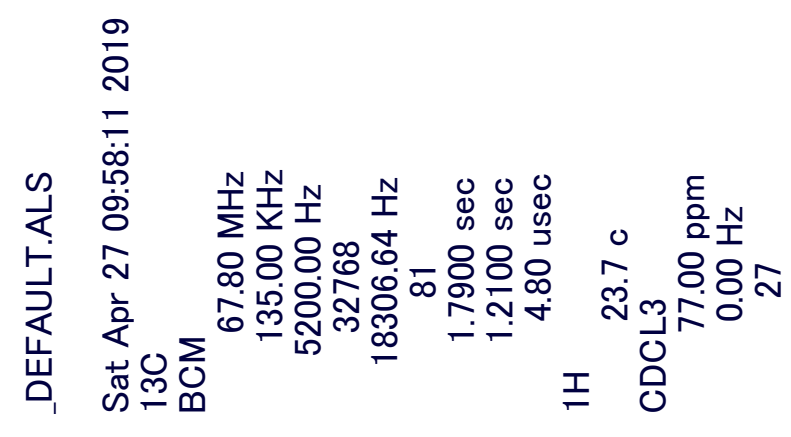

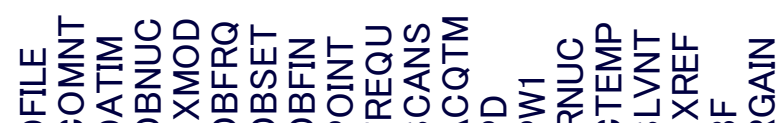

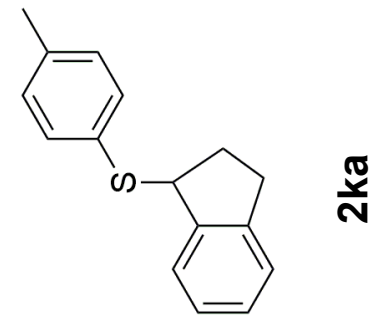

$80^{\circ} L$
$\varepsilon L{ }^{\circ} 0 \mathcal{-}$
$0 \mathcal{G}^{\circ} \varepsilon E$

$8 \varepsilon^{\prime} \mathrm{s}$

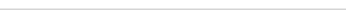

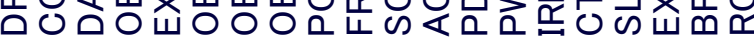

Z9॰Z!

$6 \varepsilon^{\prime} 9$ ᄂ

Z9 LZL

$\angle 9621$

96 니

乙乙乙६ ।

86. $9 \varepsilon$ เ

$\varepsilon 0^{\circ} \varepsilon t ।$

$\nabla L \cdot \varepsilon t$ 


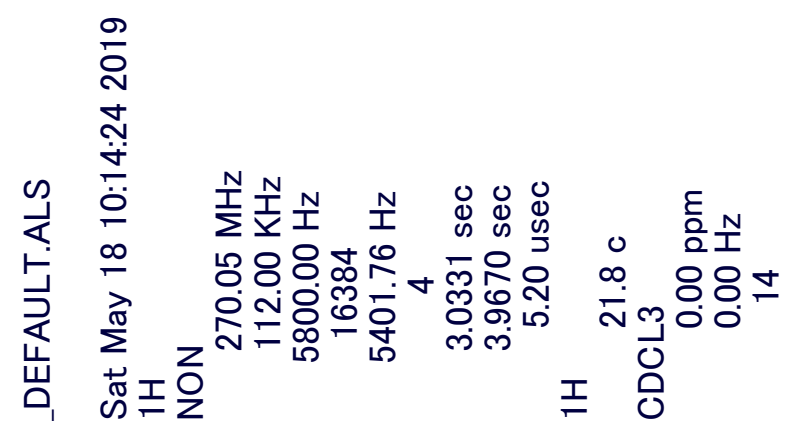

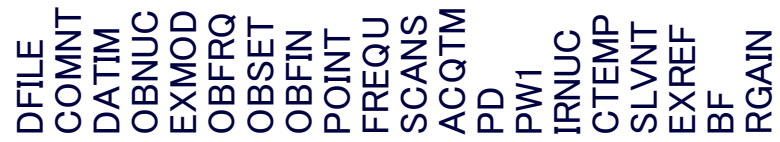
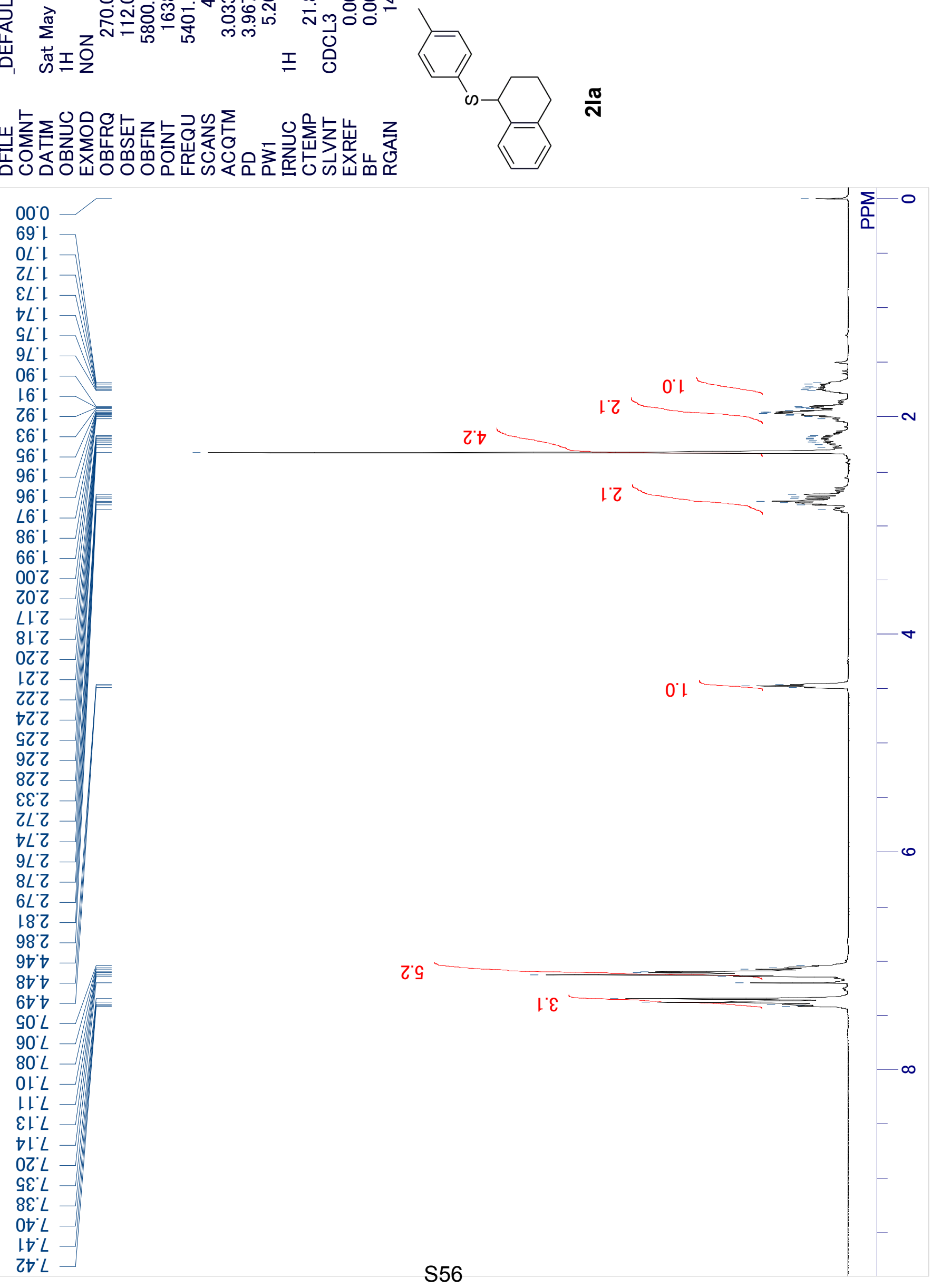


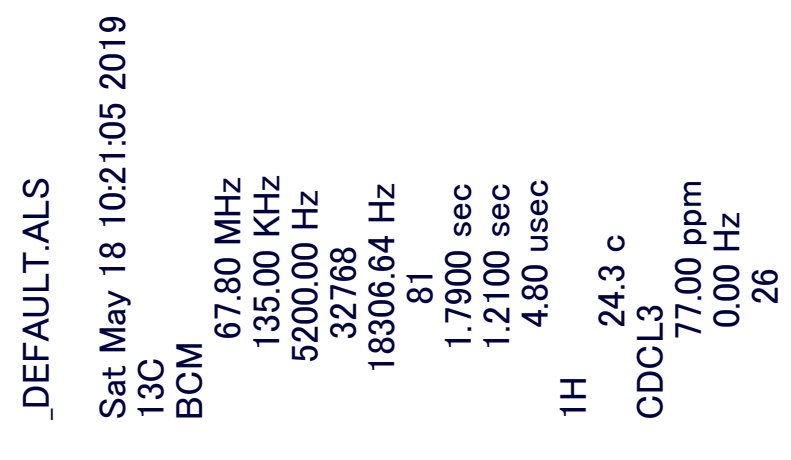

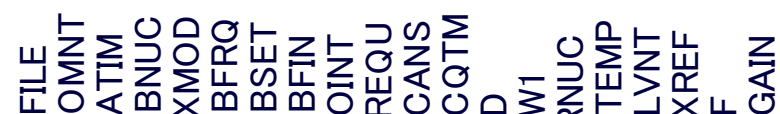

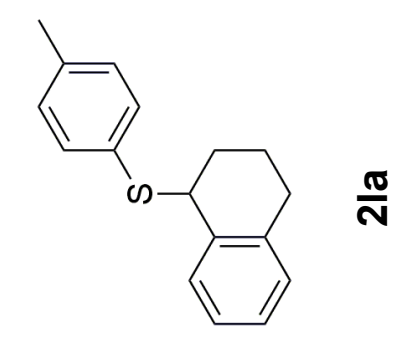

$09 \cdot 81$

60 เ

$9 \varepsilon^{\circ} 8 乙$

0162

Gl:8t

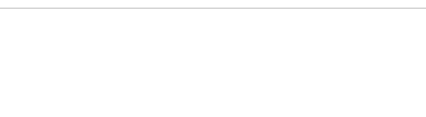

ZG.9L

$00^{\circ} L L$

$\angle \nabla^{\circ} L L$

29.9Z।

†6.9乙।

LL6ZL

$\angle 96$ '

$\angle \nabla 0 E L$

9乙'乙E L

I ZEL

†9 $9 \varepsilon 1$

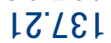

$6 t^{\circ} \angle \mathcal{E}$ 


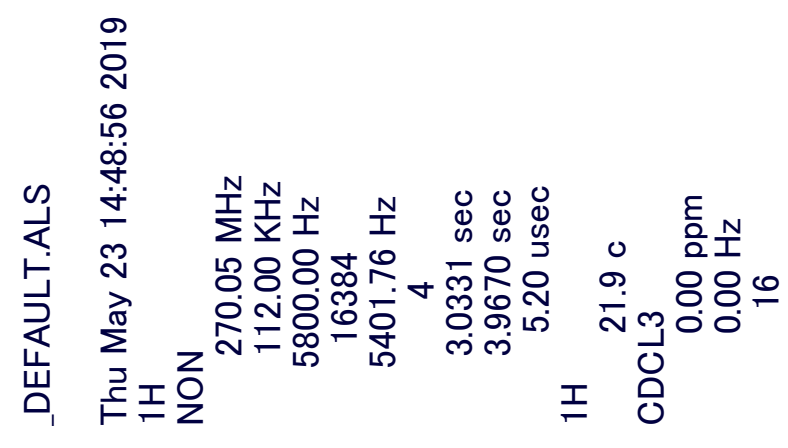

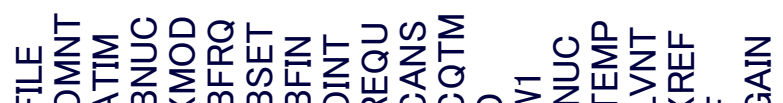

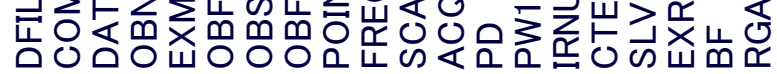

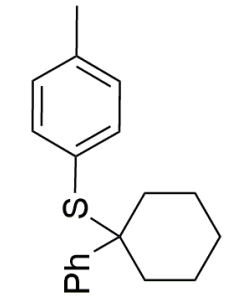

$\frac{\sigma}{N}$

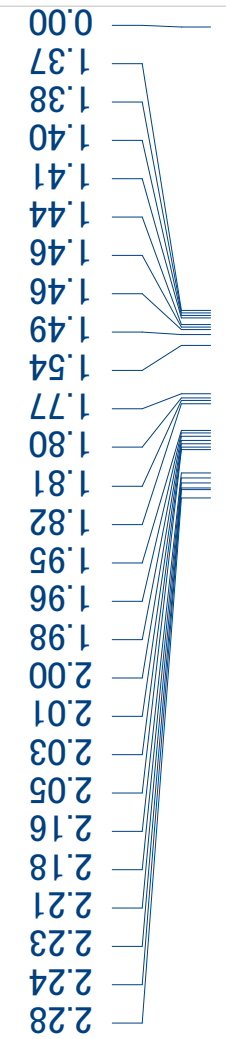

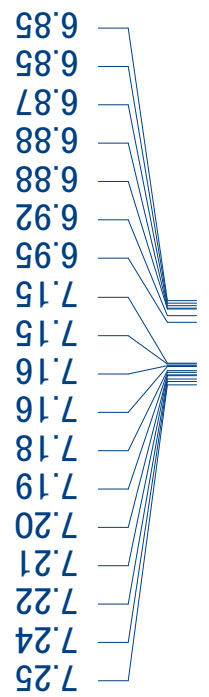

$0 " t$ 


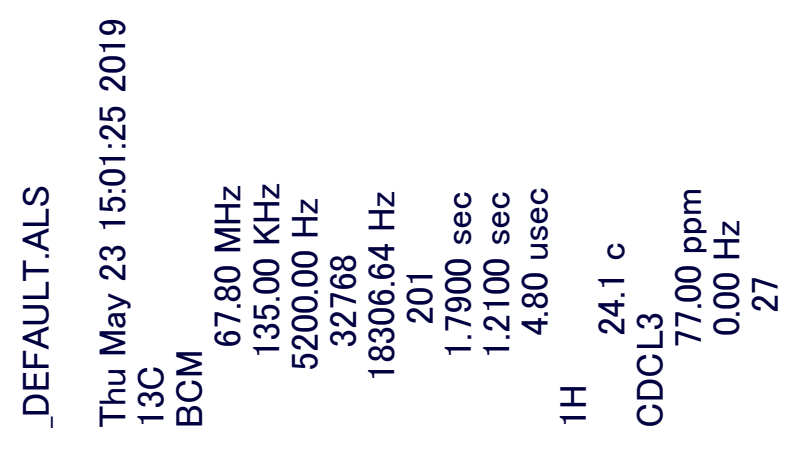

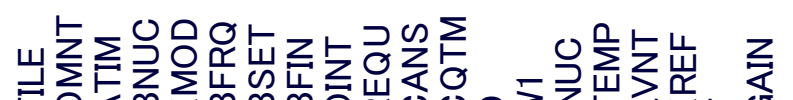

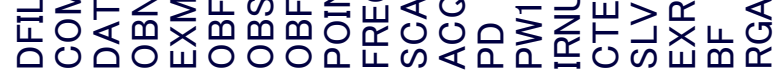

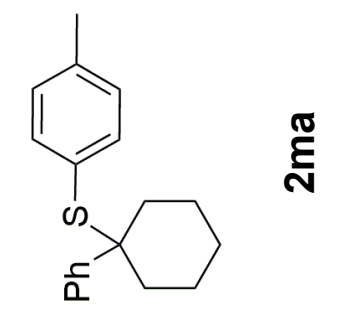

81.12

98.27

68. 92

$\left\llcorner Z^{\prime} 9 \varepsilon\right.$

0099

\&G $9 L$

00 LL

$\angle t L L$

$\varepsilon เ \cdot 9 Z \mid$

$\varepsilon 0$ LZI

I8LZ।

GI. $8 \mathrm{ZL}$

98.8ZI

$86.9 \varepsilon$ ।

${ }^{\circ} \mathrm{C} 8 \mathrm{E}$ । 


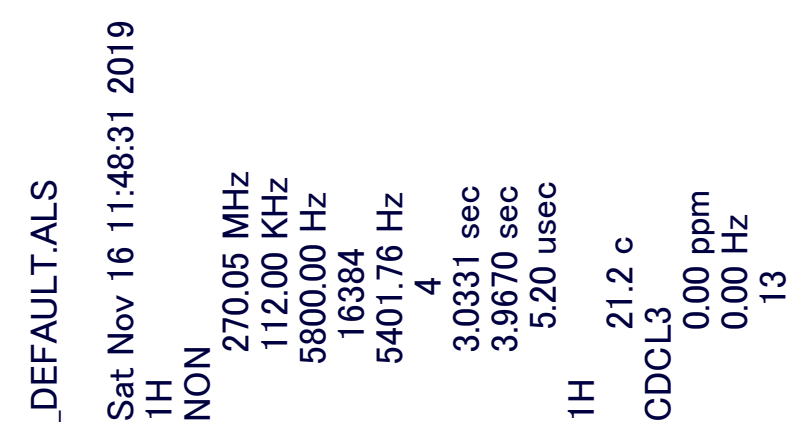

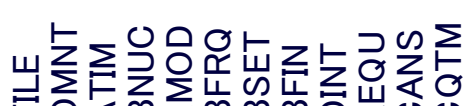

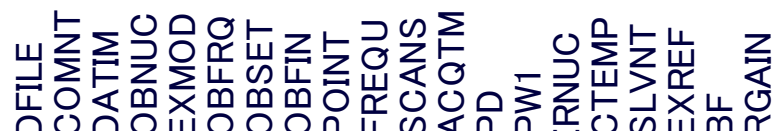

$$
00^{\circ} 0
$$

$\nabla G^{\circ}$

99.

$\varepsilon \varepsilon \cdot 乙$
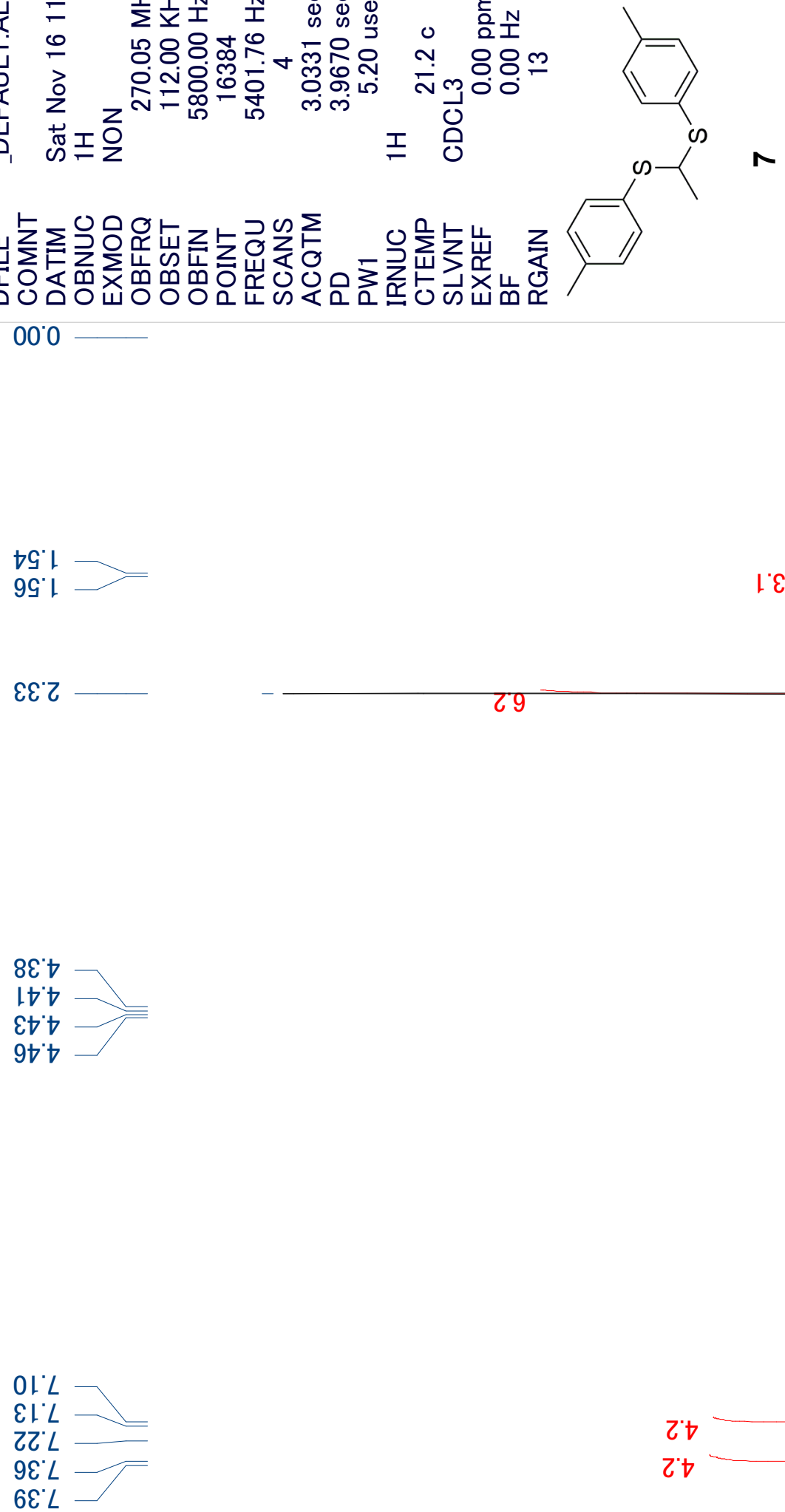


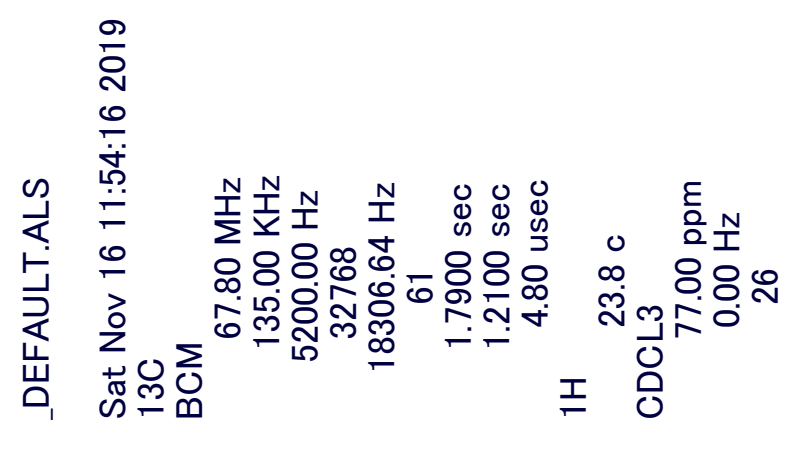

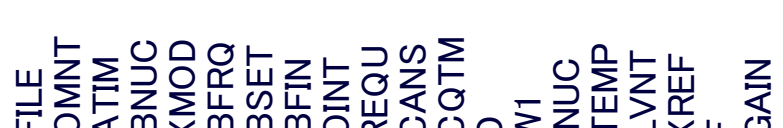

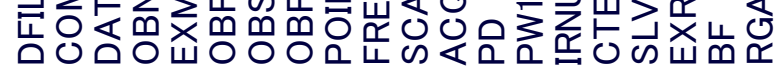
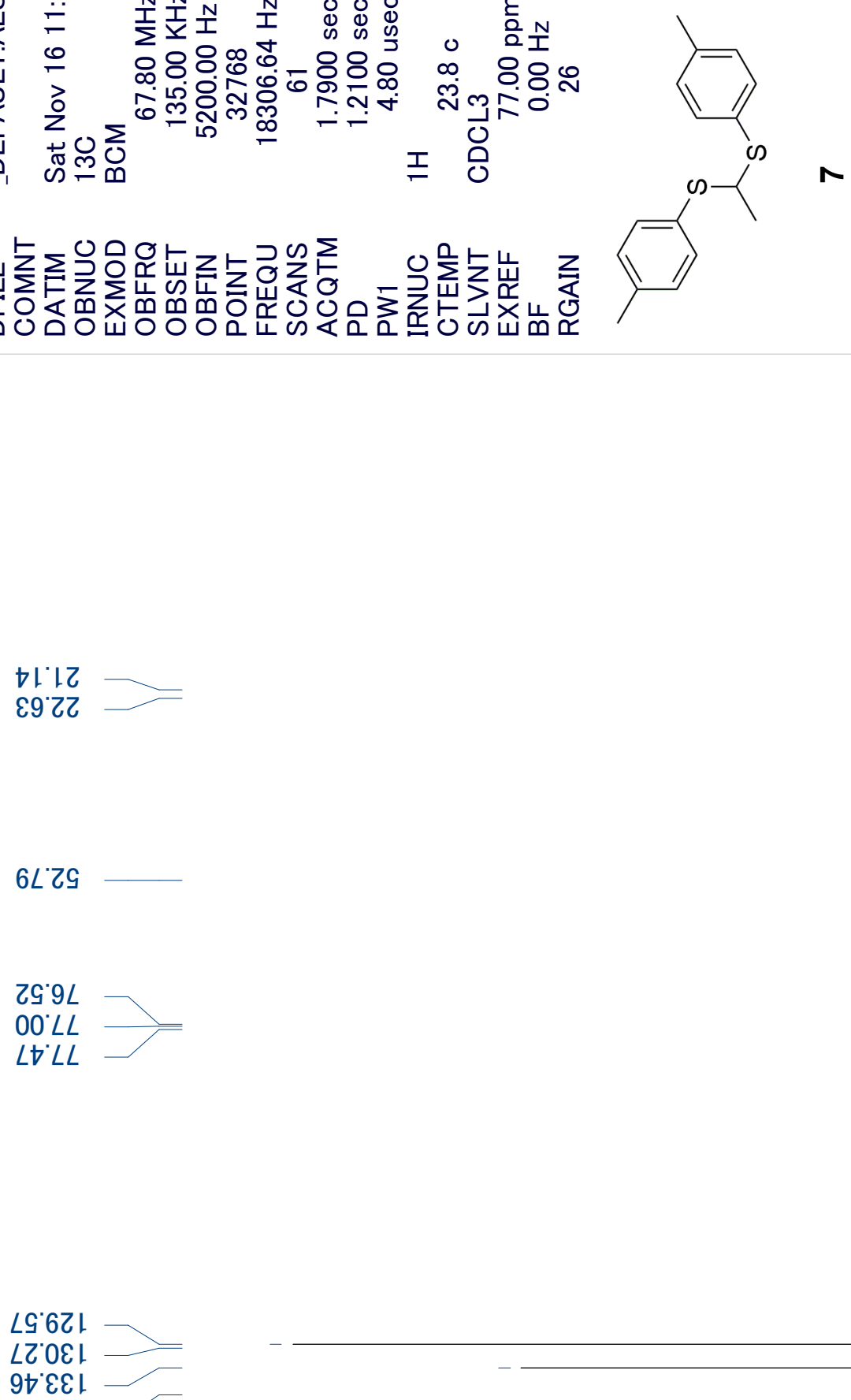

$00 \angle L$

$\angle \nabla^{\circ} L L$

$\varepsilon 6^{\circ} \angle \mathcal{L}$ L

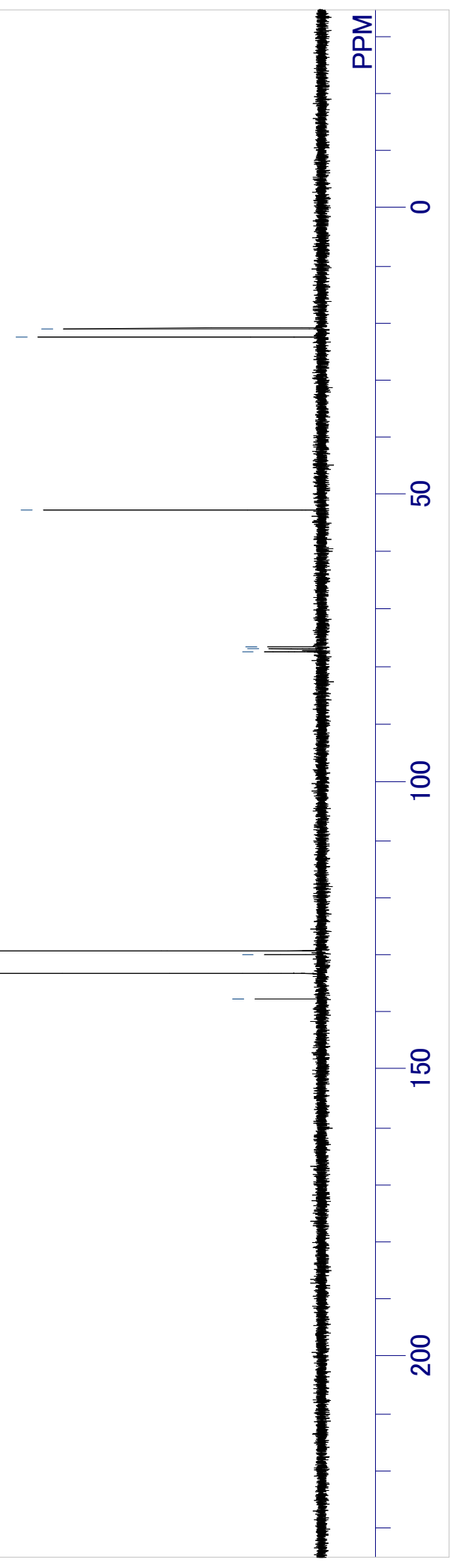

